\title{
Optical Properties of Rare Earth Nitrides
}

\author{
by \\ Muhammad Azeem
}

\section{Victoria \\ UNIVERSITY OF WELLINGTON \\ Te Whare Wānanga \\ o te Ūpoko o te Ika a Māui}

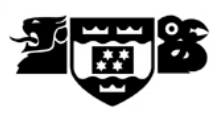

\author{
A thesis \\ submitted to Victoria University of Wellington \\ in fulfilment of the \\ requirements for the degree of \\ Doctor of Philosophy \\ in Physics.
}

Victoria University of Wellington 2013 
To my mother

Shamim Naz

For she brought me down from

heavens to earth.

And to my father

M. Naim-ur-Rehman

For he raised me high up to sky. 


\begin{abstract}
This experimental thesis uncovers the fundamental optical features of rare earth nitride compounds and relates them to their electronic structure. Experimental observations for the optical energy gaps for thin films of GdN, DyN, SmN and EuN are made for the first time. Thin films are grown by thermal evaporation in ultra high vacuum environment and are passivated by $\mathrm{MgF}_{2}$ layers. Initial characterizations indicate the polycrystalline thin films of RENs are strongly textured along [111] direction.

Optical characterization techniques, Fourier transform infrared and conventional UV/Vis spectrometers are used in conjunction with SQUID magnetometer and DC electrical resistivity. Transmission and reflection spectra for rare earth nitride thin films were obtained in the photon energy range $0.5-5.5 \mathrm{eV}$ in their paramagnetic and ferromagnetic phases. Paramagnetic GdN has a direct energy gap of $1.30 \pm 0.05 \mathrm{eV}$ which coincides well with theoretically predicted energy gap. A red - shift in the fundamental absorption edge of ferromagnetic GdN is observed along with onset of absorption at higher energy attributable to the exchange splitting of conduction and valence bands of GdN. The spin split joint density of states is in remarkable agreement with theoretically calculated spin polarized band structure of GdN. Similarly for DyN a consensus is found between theory and experiment on the energy gap of $1.20 \pm 0.05 \mathrm{eV}$ at room temperature. However, in the case of SmN, an energy gap of $1.30 \pm 0.1 \mathrm{eV}$ is underestimated by theory to $0.81 \mathrm{eV}$. For EuN, the experimentally determined value of energy gap is $0.97 \pm 0.05 \mathrm{eV}$. This value is used to tune the band structure calculation by QSGW theory which returns a ferromagnetic semiconducting solution for $\mathrm{EuN}$.
\end{abstract}




\section{Acknowledgments}

I was not alone, on this long and sinuous journey, my family was with me. They spent with me as many nights awake. Every evening, when I would return home, my four years old son, Umer, would come running to open the door for me, clutching my hands, pulling me inside, and making me forget all the distresses of the day. And my new born, Ibrahim, who just have learnt to smile, offers me a thousand reasons to cheer. Almost five year ago, Rabia, my wife pledged to stand by my side through thick and thin and she is keeping her every word since then. With her extraordinary intellectual sense, she not only has helped me to proof read my highly technical draft but also have suggested useful changes. My brother, Moazzam and sisters Aaisha and Dr. Khadija \& their families, despite being continents apart, always feel like close to me. My aunt, Musarrat Javed who had been my teacher, my mentor and my friend, all at the same time. And in the last, but by no means the least, my late father-in-law, Hafeez Ahmad, for all his prayers and encouragements.

My teachers have been my biggest source of inspiration. I remember my primary school teacher, Ghulam Yasin, whose love and affection is unforgettable for me. I also remember Dr. Khadim Hussain, Dr. Shahzad Naseem, Dr. Hassan A. Shah and Prof. Salimullah, the most revered teachers and scientists like whom I want to become.

Nearly four years ago when I landed in Wellington, the coolest little capital in the world, I was apprehensive of a different culture and loneliness. But this city and its people embraced me like an old friend. Today, while Lahore is my home city, Wellington has become the city of my heart. I want to express my greatest gratitude to the people of this wonderful city and undoubtedly the most beautiful country of the world. I also greatly appreciate the people of my host institution, Victoria University of Wellington and MacDiarmid Institute of Advanced Materials and Nanotechnology, School of Chemical and Physical Sciences, not only for the financial assistance they provided but also for their friendship which I will cherish for the rest of my life.

A challenging project such as this would never have been completed, had the assistance of several people was not available. Among the long list of my friends and colleagues, 
I am particularly thankful to Dr. Nick Strickland and Dr. Annette Koo, Industrial Research Limited, who very kindly supervised me to perform optical measurements. These measurements form the core part of my thesis. But my best compliments go to Dr. Ben Ruck and Dr. Joe Trodahl, my supervisors. I am indebted to them for giving me their precious time without any limit. They have guided me through the discussions, the arguments and the critiques. I am grateful to them for bearing with me for more than three years.

And in the last, thanks to my colleagues of spintronics group and to all who deserve but are remained to mention.

After jumping from the edge of the space, Felix Baumgartner said, "Sometimes you have to go up really high to understand how small you are." This is exactly what I am feeling now. My work has made me feel small, humble and that how little I know. I believe in God and I believe that knowledge is the key by which we can learn to recognize Him. This only is an attempt to know Him so again, I begin my humble work In the name of Allah, the most Benign, the most Compassionate. 


\section{Contents}

Abstract

Acknowledgements

iii

Table of Contents $\quad$ v

List of Figures $\quad$ vi

List of Tables vii

1 Panorama 1

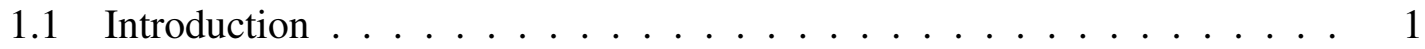

1.2 Rare-Earth Nitrides . . . . . . . . . . . . . . . . . 4

1.3 This Work . . . . . . . . . . . . . . . . . 11

2 Optical Properties and Processes in

General Electrodynamic Physical Systems

2.1 Interband Transitions and Optical Properties . . . . . . . . . . . . 15

2.2 Complex Refractive Index and Dielectric Function _ . . . . . . . . . 17

2.2.1 Relation of Transition Rate with Absorption Coefficient . . . . . 19

2.3 Determination of Optical Constants from

Transmittance and Reflectance Spectra . . . . . . . . . . . . . . 21

2.3.1 Matrix Method Using Fresnel Coefficients . . . . . . . . . . . . . 24 
2.4 TFCalc Software . . . . . . . . . . . . . . . . 27

3 Experimental Techniques $\quad 28$

3.1 Growth of Rare Earth Nitride Thin Films . . . . . . . . . . . . . . . . . . 29

3.1.1 Preparations for the Growth . . . . . . . . . . . . . . . . 29

3.1.2 Structure of UHV Growth Chamber . . . . . . . . . . . . . . 30

3.1 .3 Growth Procedure . . . . . . . . . . . . . . 32

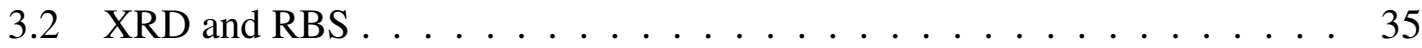

3.3 Temperature dependent resistivity and

Magnetometry. . . . . . . . . . . . . . . . . 37

3.4 Optical Measurements _. . . . . . . . . . . . . . . . . 38

3.4.1 Optical Measurements in the Infrared Region . . . . . . . . . 38

3.4 .2 Working Principle $\ldots \ldots \ldots . \ldots . \ldots 39$

3.4.3 Transmission Measurements . . . . . . . . . . . . . . . . 41

3.4.4 Reflection Measurements . . . . . . . . . . . . . . . . . . . 42

3.5 Transmittance Measurements

at Low Temperature . . . . . . . . . . . . . . . . . . . 442

3.5.1 Operational Setup . . . . . . . . . . . . . . . 42

3.6 Optical Measurements in UV/Vis Range . . . . . . . . . . . . . . . . . . 43

4 Gadolinium Nitride $\quad 45$

4.1 Preparation of GdN Thin Films . . . . . . . . . . . . . . . . 47 
4.2 Structural Analysis . . . . . . . . . . . . . . . 51

4.3 Temperature Dependent DC Resistivity

and Magnetism . . . . . . . . . . . . . . . . . . . 52

4.4 Optical Properties . . . . . . . . . . . . . . . 57

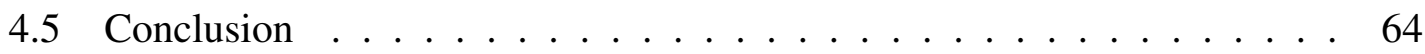

5 Low Temperature Response of GdN Transmission Spectra 65

5.1 Temperature Dependent Transmission Spectra . . . . . . . . . . . . 67

5.2 Temperature Dependent Extinction Coefficient _. . . . . . . . . 69

5.3 Conclusion .......................... 72

$\begin{array}{llr}6 & \text { Dysprosium Nitride } & 73\end{array}$

6.1 Preparation of DyN Thin Films . . . . . . . . . . . . . . . 74

6.2 Structural Characterization . . . . . . . . . . . . . 77

6.3 Temperature Dependent Resistivity and

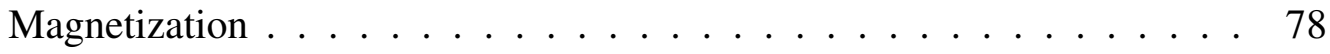

6.4 Optical Properties . . . . . . . . . . . . . . . . . . 81

6.5 Optical Spectra in the Far Infrared Region . . . . . . . . . . . . . . 89

6.6 Temperature Dependence of Transmission Spectra . . . . . . . . . . . . . 91

6.7 Conclusion . . . . . . . . . . . . . . . . . 92

7 Samarium Nitride and Europium Nitride $\quad 93$

7.1 Growth of $\mathrm{SmN}$ and $\mathrm{EuN}$ thin films $\ldots \ldots . \ldots . \ldots 93$ 
7.2 Structural Analysis . . . . . . . . . . . . . . . . . 94

7.3 Optical Spectra of $\mathrm{SmN} \ldots \ldots \ldots$. . . . . . . . . . . . 95

7.4 Optical Spectra for EuN . . . . . . . . . . . . . . . . . . . . 97

7.5 Conclusion . . . . . . . . . . . . . . . 100

8 Final Discourse $\quad 101$

8.1 Future Possibilities and Challenges f. . . . . . . . . . 103

$\begin{array}{ll}\text { Bibliography } & 104\end{array}$ 


\section{List of Figures}

$2.1 \quad$ Multiple reflection and transmission of light from a single film. . . . . . . 22

2.2 A multilayer sample. . . . . . . . . . . . . . . . . . 25

3.1 Schematic diagram for a UHV growth chamber. . . . . . . . . . . . . 31

3.2 A schematic of a rectangular van der Pauw configuration. . . . . . . . . 37

3.3 An interferogram from a GdN thin film. . . . . . . . . . . . . . . . . . . 40

3.4 Fourier Transform of interferogram signals from a GdN sample (red) and from empty slots of the sample holder (blue) to the frequency domain. . . 40

3.5 Ratios of reference spectra in frequency range $2000 \mathrm{~cm}^{-1}$ to $8500 \mathrm{~cm}^{-1}$. . 41

4.1 The band structure of GdN from Trodahl et al. ${ }^{l}$. The solid lines represent majority - spin bands, and dashed lines minority. . . . . . . . . . . . . . 46

4.2 Typical film deposition cycle. . . . . . . . . . . . . . . . . . . . . 48

4.3 Reciprocal of film resistance vs thickness for a GdN sample prepared at room temperature. . . . . . . . . . . . . . . . . . . . . . . 49

4.4 XRD pattern of a representative $G d N(G 4)$ thin film. . . . . . . . . . . . . 51

4.5 Temperature dependence of DC resistivity for GdN. The sample was prepared under the conditions similar to G6 but capped with GaN. . . . . 53

4.6 Magnetic moment of $G d N(G 6)$ as a function of temperature. . . . . . . . 54

4.7 Curie - Weiss behaviour of a $G d N(G 6)$ film. . . . . . . . . . . . . . . 55

4.8 Hysteresis loop at $5 \mathrm{~K}$ for a $G d N(G 4)$ film. Inset shows the paramagnetic

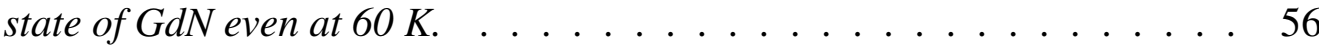


4.9 Reflectance $(R)$, transmittance $(T)$ and $R+T$ from the substrate side for the sample GdN (G5) thin film. Open circles represent simulated spectra whereas solid lines are experimental. . . . . . . . . . . . . . . . 58

$4.10 R, T$, and $R+T$ spectra from the capping side of the sample $G d N(G 5)$. . 58

4.11 Imaginary part of the complex refractive index for a $G d N(G 5)$ sample. Inset shows the absorption coefficient. . . . . . . . . . . . . . . . . . 60

$4.12 R, T$ and $R+T$ spectra from the substrate side of the film $G d N(G 1) \ldots$. . . 61

4.13 Real and imaginary parts of the dielectric function. . . . . . . . . . . . . 62

5.1 An expanded view of the band structure of $G d N$ calculated $^{1}$ by the $L S D A+U$ approach. The solid red lines represent majority spins and dashed blues lines represent the minority states.

5.2 Transmission spectra at different temperatures from a $G d N(G 6)$ sample.

5.3 Temperature dependent spectra for $G d N-G 4$ and $G 5$ samples. . . . . . . . 68

5.4 Extinction coefficient of $G d N(G 6)$ in its ferromagnetic phase at $5 \mathrm{~K}$ and in its paramagnetic state at $140 \mathrm{~K}$. . . . . . . . . . . . . . . . . . . . . 69

5.5 Evolution of imaginary part of the dielectric function with temperature for a GdN sample. . . . . . . . . . . . . . . . . . . . . . 70

5.6 Energy difference between minority and majority spin states for ferromagnetic GdN.

6.1 Band structure of DyN calculated by Larson et al. ${ }^{2}$ The dashed and solid blue lines are for majority and minority spins. Red solid and dashed lines represent filled and empty 4 fevels. Inset shows an enlarged view of the direct energy gap at the X point. . . . . . . . . . . . . . . 73 
6.2 Reciprocal of film resistance vs thickness for DyN(D5) establishes uniform growth of the film prepared at room temperature. . . . . . . . . . . . 76

6.3 XRD pattern for a representative DyN(D5) sample. . . . . . . . . . . . . 77

6.4 Temperature dependent DC resistivity of a DyN thin film with a GaN cap, grown under the similar conditions as of DyN(5). . . . . . . . . . . . . 78

6.5 Magnetization as a function of temperature for a DyN(D4) sample. . . . . 79

6.6 Hysteresis curves at low temperatures for the sample DyN(D5) which is

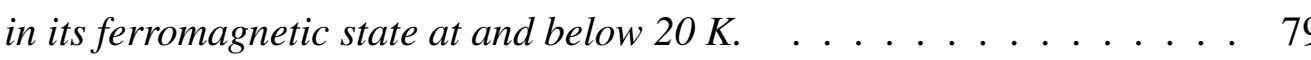

6.7 Curie - Weiss plot for a DyN(D3) sample. . . . . . . . . . . . . . . 80

$6.8 R, T$ and $R+T$ from the substrate side of $D y N(D 5)$ thin film. Solid lines are experimentally obtained spectra whereas open circles represent fitted

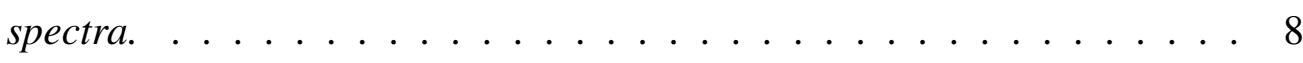

$6.9 R, T, R+T$ from the cap side of a $D y N(D 5)$ thin film. . . . . . . . . . . 82

$6.10 R, T \& R+T$ spectra of a DyN (D3) film with nitrogen vacancies. The solid lines are experimental data and fitting is represented by open circles. 84

$6.11 R, T \& R+T$ from the cap side of $D y N(D 3)$ thin film. . . . . . . . . . . 84

6.12 Imaginary part of the complex refractive index for a near stoichiometric DyN(D5) film against photon energy showing the onset of absorption at around $1.2 \pm 0.05 \mathrm{eV} \ldots \ldots \ldots \ldots$. . . . . . . . . . . 86

6.13 Absorption coefficient for DyN samples with significant levels of nitrogen vacancies. . . . . . . . . . . . . . . . 86

6.14 Free carrier absorption (black solid circles) and the optical gap (blue solid squares) vs. $N_{2} /$ Dy flux ratio during growth. . . . . . . . . . . . . . 87

6.15 Real and Imaginary parts of the dielectric function. . . . . . . . . . . . . 88 
6.16 Infrared reflectivity spectrum of a $\mathrm{Y}$-stabilized $\mathrm{ZrO}_{2}$ substrate and a DyN thin film on a YSZ substrate capped by amorphous Si along with a fit to the .90

6.17 Fitted real and imaginary parts of the complex dielectric function of DyN showing the polar phonon and nitrogen - vacancy donor to conduction band transition. . . . . . . . . . . . . . . . . . . . 990

6.18 Temperature dependence of the absorption edge in DyN(D5) thin films. . 91

7.1 XRD spectrum for a SmN thin film. . . . . . . . . . . . . . . . . . . . 94

7.2 Reflectance $(R)$, Transmittance $(T)$ and their sum $(R+T)$ measured from a SmN film. . . . . . . . . . . . . . . . . 95

7.3 Plot of extinction coefficient versus energy for SmN. Absorption coefficient (inset) rises above $10^{4} / \mathrm{cm} \ldots \ldots . \ldots . \ldots 96$

7.4 Reflectance and transmittance and their sum for thin film of EuN in the

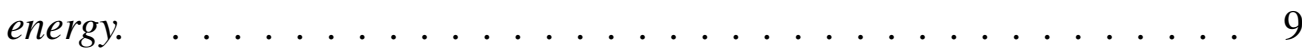

7.5 Energy dependent absorption coefficient for a EuN thin film estimated from the room temperature transmission spectrum indicating a fundamental absorption edge around $0.97 \pm 0.05 \mathrm{eV} \ldots \ldots$. . . . . . . . . . 98

7.6 A ferromagnetic semiconducting band structure for EuN calculated by QSGW theory by Richter et al. ${ }^{3} \ldots$. . . . . . . . . . . . . . . . 99 


\section{List of Tables}

3.1 Physical Properties of Rare Earth Metals and Boat Materials _ . . . . . . 29

3.2 Frequency Range of FTIR . . . . . . . . . . . . . . . . . 39

4.1 Growth Parameters of GdN Thin Films grown at Room Temperature . . . 50

6.1 Growth Parameters of DyN Thin Films grown at Room Temperature . . . 75

6.2 Effect of Nitrogen Vacancies on the Fundamental Absorption Edge . . . . 85 


\section{SECTION 1}

BACKGROUND 


\section{Chapter 1}

\section{Panorama}

\subsection{Introduction}

The advanced features carried by the modern electronic devices are principally due to the progress made in conventional semiconductors like silicon and germanium, where the charge degree of freedom of electrons has been manipulated. But the human yearn to dig deep is now making it to look farther than the electronic charge. Currently scientists are in quest of possible applications of another cognate property of electrons - its spin. ${ }^{1-3}$

Subtle characteristics of electron spin, which inherently is a quantum mechanical phenomenon, could potentially be exploited in electronic devices. Unlike electronic charge, which may be scattered due to collisions with defects, impurities and or other charges, a spin has a long quantum coherence time. ${ }^{4,5}$ Information can be carried by the spin transport ${ }^{6}$ and it can be manipulated optically or by applying magnetic fields. Spin of an electron can assume an "up" or "down" state as if a binary logic and the spin angular momentum of an electron is never zero.

These characteristics of the spin degree of freedom of an electron are a matter of hot pursuit. Combined utilization of the spin and charge of an electron, so called the subject of spintronics, has opened up new possibilities to improve the functionality and efficiency of electronic devices. ${ }^{7}$ Non volatile magnetic random access memory (MRAM) ${ }^{8-10}$ and magnetic hard disks ${ }^{11,12}$ provide the users exceedingly high data storage capacity. The field is further enriched with the inclusion of magnetoelectronics. Multilayer ferromagnets show giant magnetoresistance $(\mathrm{GMR})^{13,14}$ or tunnelling magnetoresistance (TMR) and do not need an external magnetic field. ${ }^{15-21}$ Spin field effect transistors ${ }^{22-24}$ and spin-logic devices ${ }^{25,26}$ are the results of these investigations.

Moreover, an understanding of spin interaction in protein molecules ${ }^{27}$ is being developed which would pave the way to the complex computational devices. ${ }^{28,29}$ Spin chemistry ${ }^{30}$ 
has emerged, relating spintronics with nuclear spin effects. Spintronics, therefore, is swiftly changing from fundamental studies of electron spin in condensed matter to engineering and nanospintronics. ${ }^{31}$ It seeks its applications in technologically advanced and intricate device fabrication of atomic specifications.

Despite a continuing expansion in ferromagnetic devices, endeavours made in semiconductor spintronics are limited because of the nonmagnetic nature of conventional semiconductors. However, it has been recognized that doping of magnetic elements like Mn would impart magnetic properties to the semiconductors. Thus is obtained a new class of materials, known as dilute magnetic semiconductors (DMS), where coupling between spin degrees of freedom and charge exists. In DMS, magnetic constituents come from the family of transitions metals (TM) or rare earths (RE). The magnetic behaviour of these elements is due to partially filled $d$ states in transition metals and $f$ states in rare earth metals which hybridize with $p / s$ states of host materials. The magnetic moments of dopants then interact via host bands resulting in ferromagnetism. ${ }^{32}$ For example, ferromagnetism is induced in III - V compounds, (In,Mn)As and (Ga,Mn)As, due to the magnetic interaction of free carriers and Mn spins. ${ }^{33-35}$

Nitride semiconductors doped with magnetic elements also have gained much attention as potential spintronic materials. Comprehensive reports on $\mathrm{AlN}$ and $\mathrm{GaN}$, doped with TM $(\mathrm{Mn}, \mathrm{Cr}, \mathrm{Co}, \mathrm{Fe}, \mathrm{Cu}, \mathrm{V})^{36-44}$ and RE $(\mathrm{Gd}, \mathrm{Eu}, \mathrm{Dy}, \mathrm{Er})^{41,45-53}$ can be found in the literature. Many of these studies report a high $\mathrm{T}_{C}$ value attributed to the double exchange $^{32}$ interaction of hopping electrons within $d$ bands of the dopant, but it also has been acknowledged that presence of secondary phases might be responsible for the occurrence of ferromagnetism. For example in $(\mathrm{Ga}, \mathrm{Mn}) \mathrm{N}$, a phase of $\mathrm{MnN}$ is detected ${ }^{54-56}$. Similar precipitation of $\mathrm{Gd}$ in $\mathrm{GaN}$ is also observed. ${ }^{48}$

In contrast, the presence of carriers is not related to the ferromagnetic order in concentrated magnetic semiconductors (CMS) which encompasses Eu chalcogenides (EuS and $\mathrm{EuO}$ etc. $)^{57,58}$ and $\mathrm{Cr}$ chalcogenide - spinels $\left(\mathrm{CdCr}_{2} \mathrm{~S}_{4}, \mathrm{CdCr}_{2} \mathrm{Se}_{4}\right.$ etc. $)$ and lead chalcogenides. ${ }^{20,59}$ Europium chalcogenides are efficient spin filters ${ }^{60-62}$ in metal/magnetic insulator/metal heterostructures but their applications are limited due to 
their low $\mathrm{T}_{C}$. Curie temperatures of chalcospinels are somewhat higher e.g. for $\mathrm{CuCr}_{2} \mathrm{Se}_{4-x} \mathrm{Br}_{x}$, it exceeds room temperature ${ }^{20}$ but the material is not stable in the atmosphere. Ferromagnetic semiconducting compounds $\mathrm{CuCr}_{2} \mathrm{~S}_{4}$ and $\mathrm{MnCr}_{2} \mathrm{~S}_{4}$ have $\mathrm{T}_{C}$ above room temperature ${ }^{20,63}$ however these are not very pure to be used in electronic applications.

Mixed valence manganites, $\left(\mathrm{R}_{1-x} \mathrm{~A}_{x}\right) \mathrm{MnO}_{3}(\mathrm{R}=$ rare-earth cation, $\mathrm{A}=$ alkali or alkaline earth cation) ${ }^{64}$ and related materials are magnetic semiconductors and exhibit colossal magnetoresistance ${ }^{65}$ with ferromagnetic order beginning at $350 \mathrm{~K}$.

Possible alternatives to DMS and CMS are transition metal silicides. An alloy $\mathrm{Fe}_{1-x} \mathrm{Mn}_{x} \mathrm{Si}$ is p-type paramagnetic. ${ }^{66}$ Metallic magnetic compounds $\mathrm{Fe}_{1-x} \mathrm{Co}_{x} \mathrm{Si}$, which retain the crystal structure of FeSi, share similar magnetization with $(\mathrm{Ga}, \mathrm{Mn}) \mathrm{As}$. Both are affected by the magnetic fields in similar fashion in spite of difference in $\mathrm{T}_{C}$ values, $53 \mathrm{~K}$ for $\mathrm{Fe}_{1-x} \mathrm{Co}_{x} \mathrm{Si}$ and $110 \mathrm{~K}$ for $(\mathrm{Ga}, \mathrm{Mn}) \mathrm{As}$. However incorporation of nonmagnetic silicon reduces the conductivity and also affects the crystal structure and multiphase composition of the sample. ${ }^{20}$

In spite of great advances made, use of those initial spintronic materials is far from realization in the electronics industry. Common ferromagnetic materials are metals and non-metallic magnetic materials are antiferromagnets or ferrimagnets. In DMS, (and also in CMS) precipitation of magnetic dopants, independent control of carrier concentration without afflicting their magnetism, unstable crystal structure and conduction are the common attributes which prevent the assembly of ferromagnetic materials with the right combination of semiconducting, magnetic and optical properties. The business of microelectronics still relies heavily on the alloys of $\mathrm{Co}-\mathrm{Pt}, \mathrm{Sm}-\mathrm{Co}, \mathrm{Fe}-\mathrm{Ni}$ to supply storage disks, reading heads and process chips.

In the middle of the 20th century, commercialization of solid-state electronics faced the similar circumstances once germanium was not able to fulfil the requirements. It had been solely the replacement of germanium with silicon that lead to a revolution in the business of electronics. The time is ripe and the stage is set for a new type of materials to be discovered. This is the background against which the present work was conducted and 
this is the panorama where nitride compounds of rare earths enter the spintronic vista.

\subsection{Rare - Earth Nitrides}

Rare earth metals, one of the most abundant elements on the earth, are finding out demand within international market due to their magnetic properties. Due to an almost identical outer shell, the electronic configuration rare earth (RE) elements are very similar chemically. ${ }^{67}$ But a different electron occupation number for the shallow inner $4 f$ shell imparts in rare earth elements and their compounds a wide range of magnetic properties.

The number of $4 f$ electrons increases from 0 to 14 through the series, lanthanum to lutetium. The highly localized nature of $4 f$ levels prohibits direct interaction between $f$ - $f$ states of neighbouring RE atoms therefore they form significantly narrow bands compared to $s, p$ and $d$ states which are broader. ${ }^{68}$ Due to onsite Coulomb repulsion, the energy difference between occupied and unoccupied $4 f$ levels is quite large. Lack of direct orbital overlap of $4 f$ electrons on neighbouring ions means that the exchange interaction that leads to the magnetic ordering can be quite complex. ${ }^{69}$ This is in contrast to the more itinerant nature of $d$ electrons in transition metals. The spin-orbital moment is particularly strong for many of the rare earth elements because $f$ orbital moments generally are not quenched by the crystal field. ${ }^{68}$ In general magnetic transition temperatures for rare earth pnictides (rare earth compounds with $\mathrm{N}, \mathrm{P}, \mathrm{As}, \mathrm{Sb}$ and $\mathrm{Bi}$ ) are smaller than for $3 d$ transition metal elements or compounds because the inner shell magnetic moments are largely aligned through intra - atomic $s(d)$ - $f$ exchange interaction and weaker inter atomic $s-s(d-d)$ exchange interactions.

Monopnictides of this family of elements form a simple fcc rock salt $(\mathrm{NaCl})$ crystal structure $^{69-71}$ where each RE atom coordinates with 6 of its neighbouring atoms. The bonds have ionic character. The experimental lattice constant ${ }^{72-75}$ is usually different from the calculated ${ }^{76}$ by $1-2 \%$. Nitride compounds of rare earth metals are particularly attention - grabbing materials. This category contains intrinsic ferromagnetic semiconductors to half metals and thus have potential to become spintronic materials. 
The preparation of lanthanide nitrides is especially difficult due to their propensity to react with oxygen. So in order to understand the properties, stoichiometric samples of rare earth nitrides with the low defect density are needed. Earlier reports suggest that the bulk rare earth nitride samples were prepared by a variety of methods involving the direct reaction of rare earth metals with nitrogen in an arc furnace or by synthesis of hydrides followed by reaction with ammonia to make the nitrides. ${ }^{77-83}$ In the mid 80 s and early 90 s, successful attempts to grow large single crystal $\mathrm{ScN}$ and $\mathrm{YbN}$ were made. ${ }^{84-88}$ Single crystals of $3 \mathrm{~mm}$ to $5 \mathrm{~mm}$ edge length had a lattice constant of $4.781 \AA$ A. The compound was found to be stoichiometric within $\pm 0.5 \%$. A non-stoichiometric compound has comparatively large conduction ${ }^{89}$ as nitrogen vacancies add free electrons to the system.

A thin film is the most likely type for the technological applications. Considerable advances have been made to grow polycrystalline and epitaxial films of rare earth nitrides in last few years by using ultra high vacuum technique. Molecular beam epitaxy (MBE) $)^{3,74,75,90-92}$, pulsed laser deposition (PLD) $)^{91-96}$ and dc/rf magnetron sputtering ${ }^{95,97-99}$ are the common techniques used to obtain high quality thin films of RENs in the presence of pure nitrogen gas. Growth of EuN requires ionized nitrogen provided by the Kaufman source, a compact ion source based on electron bombardment of $\mathrm{N}_{2}$ plasma. ${ }^{91-93}$

Perhaps the simplest preparation technique involves evaporation of RE metallic ingots either thermally ${ }^{100,101}$ or by an electron gun in a background nitrogen partial pressure of $10^{-5}$ to $10^{-4}$ mbar where the substrate is held at room temperature. Substrate choice depends on the characterization techniques intended. For the epitaxial growth $\mathrm{MgO}(100)$ has been used ${ }^{74,102}$ but it is found that once the film thickness exceeds $60 \mathrm{~nm}$, its surface quality gets affected. Silicon on the other hand reacts with RE elements to form disilicides ${ }^{103}$ therefore buffer layers are needed between film and substrate, adding an extra complication in the process of growth. Yttria stabilized zirconia (YSZ) is the most closely lattice matched substrate. ${ }^{91}$ However if the growth is required at high temperatures, an oxide layer of rare earths is formed at the film/substrate interface because of the mobility of oxygen in YSZ. In order to protect the films in an open atmosphere, a number of capping layers are being used. Again the choice strongly depends on the 
ex - situ measurements. Metallic overlayers of $\mathrm{W}, \mathrm{Cr}, \mathrm{Cu}$ along with $\mathrm{AlN}, \mathrm{GaN}$ and $\mathrm{MgF}_{2}{ }^{69,100,101,104-107}$ have been used in the past. Samples need to be stored in a vacuum or an inert gas environment which helps to ensure the sample purity and the interface quality.

There are contradictions about the magnetic character of rare earth nitrides in earlier experimental reports. For example Wilkinson et al. ${ }^{79}$ showed by neutron diffraction investigation that $\mathrm{HoN}$ and $\mathrm{TbN}$, both, are ferromagnetic with Curie temperatures of $18 \mathrm{~K}$ and $43 \mathrm{~K}$ respectively whereas Busch et ${ }^{107}$ al considered $\mathrm{TbN}$ a ferrimagnet and Bommeli et al. ${ }^{108}$ an antiferromagnet $\left(\mathrm{T}_{N} 31 \mathrm{~K}\right)$. Then there were reports for $\mathrm{YbN}$ to be a semiconductor ${ }^{109}$ and semimetal. ${ }^{87,110}$ Similarly the physical properties of LaN were also contentious. $^{72}$

Modern band structure calculations predict that this class of materials comprises of magnetically ordered half-metals (with an energy gap at the Fermi level in either $\uparrow$ or $\downarrow$ electrons) and semiconductors. ${ }^{69,111}$ Discovery of theoretical methods such as augmented plane wave method (APW), density functional theory (DFT), density mean field theory (DMFT) and local spin density approximation (LSDA) in conjunction with the enlarged computational power created the potential to perform advanced band structure calculations. ${ }^{112,113}$ These methods, once applied to the rare earth nitrides systems ${ }^{111,114-120}$ highlighted the nature of their ground state.

In a simple model a trivalent rare earth ion would donate two $6 s$ electrons along with a $5 d$ or a $4 f$ electron to leave nitrogen in -3 state. Therefore the valence band is derived from occupied N $2 p$ states while the empty $5 d$ and $6 s$ rare earth states form the conduction band. Also one would expect, in this model, for $4 f$ states to lie close to the Fermi level. Such a band structure, indeed, was the result of initial calculations by using local spin density approximation (LSDA) in density functional theory (DFT). ${ }^{68}$ However this model ignores the strong correlation among $4 f$ electrons. Also the local spin density approximation fails for materials possessing a band gap. ${ }^{113}$ Furthermore two different spin orientations should be treated separately, a requirement for the strong exchange interaction between $4 f$ and band electrons. Use of the local spin density approximation with self - interaction correction (SIC-LSDA) predicted ${ }^{111}$ the ground state of RENs from metallic to insulating. 
According to this study PrN and GdN were half - metallic in their ground states. The local spin density approximation in density functional theory with Hubbard $U$ corrections $(\mathrm{LSDA}+U)$ concluded that $\mathrm{PrN}, \mathrm{PmN}, \mathrm{EuN}$ and $\mathrm{SmN}$ are half - metallic while other RENs possess small indirect band gap that tend to increase as the rare - earth atomic number increases. ${ }^{2}$

The magnetic and electronic transport properties of lanthanide compounds has been in focus for many decades. Nitride compounds of lanthanides seem to favour ferromagnetic order at low temperature $58,79,86,104,121-128$, however a small amount of oxygen causes paramagnetism and short range - antiferromagnetism. ${ }^{129-132}$ The origin of their ferromagnetism, though, is debatable. ${ }^{126,133}$ On the other hand, antimonides are metamagnets and other compounds are antiferromagnetic. ${ }^{73,134-136}$ The stoichiometry is also found to affect the magnetic ordering temperatures in rare earth compounds. For example, in GdN systems reported values of $\mathrm{T}_{C}$ differ greatly from $30 \mathrm{~K}^{97}, 50 \mathrm{~K}^{101}, 70 \mathrm{~K}$ to as high as $300 \mathrm{~K} .{ }^{137}$ A detailed analysis of magnetic ordering suggests that there exist two competing exchange interactions in GdN. The first is an antiferromagnetic Gd - Gd superexchange contribution mediated through the nitrogen ions and the second is a ferromagnetic Gd - Gd interaction mediated by the Gd $d$ orbitals. A Ruderman, Kittel, Kasuya, Yosida (RKKY) type carrier mediated exchange is considered in Gd monopnictides. However whether this mechanism is also applicable to the semiconducting $\mathrm{GdN}$ is still not clear.

An experimental insight into the magnetic properties of rare - earth nitrides is obtained by using the techniques like super conducting quantum interference device (SQUID) magnetometer, vibrating sample magnetometer (VSM), ferromagnetic resonance (FMR) and $\mathrm{x}$ - ray magnetic circular dichorism (XMCD). In XMCD, the $\mathrm{M}_{4,5}$ absorption edges in the soft X-ray spectral ranges probe the electronic transitions to the empty $4 f$ states. In the hard X-ray spectral range, the $\mathrm{L}_{2,3}$ absorption edges probe the transitions to the empty $5 d$ states which may be polarised through intra - atomic $f$ - $d$ exchange interaction.

GdN has been a testing ground to model and understand the exchange mechanism in RENs because it has a half filled $4 f$ shell and therefore no orbital component to the $4 f$ magnetism. Leuenberger et al. ${ }^{138}$ measured $\mathrm{X}$ - ray magnetic circular dichroism 
spectra at the $\mathrm{Gd} \mathrm{L}_{2,3}(2 p \rightarrow 5 d)$ absorption edges in $\mathrm{GdN}$ layers to investigate the $4 f$ induced magnetic polarization of the Gd $5 d$ derived conduction band states. Those data measured the conduction band maximum relative to the $\mathrm{Gd} 2 p$ core level. A very high ratio of the amplitudes of the dichroic signals, $\left|L_{3} / L_{2}\right|$ was observed by the authors. This was mainly due to a reduced $\mathrm{L}_{2} \mathrm{XMCD}$ signal. The authors found that $\mathrm{Gd}-5 d t_{2 g}$ states experience exchange splitting below $\mathrm{T}_{C}$. The Curie temperature of GdN layers in their work was smaller, around $30 \mathrm{~K}$, indicating a reduced exchange interaction, the mechanism responsible for ferromagnetic order. Also their polycrystalline GdN films reportedly exhibited good stoichiometry and a nearly $1.5 \%$ extended lattice parameter. The exchange splitting of $5 d t_{2 g}$ states lead to a significant shift in the $\mathrm{L}_{2}$ - edge to lower energies while the $\mathrm{L}_{3}$ - edge remained unchanged. The $\mathrm{L}_{2}$ absorption edge is essentially related to $5 d t_{2 g}$ states with the strong $\mathrm{j}=3 / 2$ character. The $\mathrm{L}_{3}$ - edge, in contrast, is associated with the measurement of $5 d e_{g}$ states due to their $\mathrm{j}=5 / 2$ character. Another successful modelling of XMCD analysis, based on LSDA $+U$, predicted half - metallic behaviour in $\mathrm{GdN} .{ }^{139,140}$

In general, $\mathrm{GdN}$ is considered to be a soft magnetic material, however the strain phases in a sample may lead to its magnetic hardening. Recent studies have reported a coercive field of several hundred Oe in GdN. ${ }^{130,132}$

Initial theoretical studies proposed a half-metallic ${ }^{141}$ and then the semiconducting ${ }^{116}$ nature of GdN. The band structure computed by LSDA $+U$ has flat bands of occupied majority $4 f$ spins, about $7.0 \mathrm{eV}$ below the Fermi level while the location of minority $4 f$ spin states was $5.0 \mathrm{eV}$ above the Fermi level. Further, the conduction and the valence bands show strong but an opposite exchange splitting of around $0.5 \mathrm{eV}$. So the conduction band minimum and the valence band maximum, both, have a majority spin character.

Another theoretical model ${ }^{142}$, however, found that the Gd $5 d$ and N $2 p$ states carry equal and opposite moments so the material is inherently antiferromagnetic. According to this model hybridisation between $5 d$ and $2 p$ states is for the majority spins so they lie close together and the transfer of majority $d$ spin leaves net minority spin at nitrogen sites. But a strong $f-d$ coupling on the Gd sites aligns $4 f$ moments with $5 d$ moments causing an 
overall ferromagnetism amongst the $4 f$ spins. However this model indicates a very low $\mathrm{T}_{C}$ value of $11 \mathrm{~K}$.

Other members of the rare earth nitride family are as interesting. Samarium has two electrons less than the half filled $4 f$ orbital while europium is one electron shorter. The trivalent samarium ion has a spin angular momentum $S=5 / 2$ and an orbital angular momentum $\mathrm{L}=5$. Due to a small energy separation between the ground and the lowest excited state multiplets, a net magnetic moment is given by $M=\left(L_{z}+S_{z}\right) \mu_{B}<<$ $\mu_{B}{ }^{71,91,143}$ Therefore the spin and the angular momenta cancel each other because the spins, although ferromagnetically aligned, are opposed to orbital moment. This feature is also a highlight of the $\mathrm{SmN}$ band structure ${ }^{2}$ calculated using $\mathrm{LSDA}+U$ where five majority spin occupied $f$ bands occur in four narrow energy ranges. The empty majority and minority bands are about $1 \mathrm{eV}$ above and 3 to $8 \mathrm{eV}$ below the Fermi level respectively. Majority bands significantly hybridize with the rest of the conduction band. Whereas selfinteraction-corrected local spin density (SIC - LSD) theory predicted a half metallic band structure $^{111}$ for SmN with a spin down energy gap of $1.29 \mathrm{eV}$ and an overlap of spin up bands. Although an expected zero total moment is so far not observed, a substantially smaller than free ion moment of $0.71 \mu_{B}$ has been reported previously. ${ }^{71,112,144-147}$ Recent experimental studies on thin films of $\mathrm{SmN}$ point towards a ferromagnetic order ${ }^{71,112}$ with a Curie temperature of $27 \pm 3 \mathrm{~K}$. In its ferromagnetic order, a hysteresis loop exhibits a large coercive field with a very small magnetic moment of around $0.035 \mu_{B} / \mathrm{Sm}^{3+}$ at a temperature as low as $2 \mathrm{~K}$ in an applied field of $0.5 \mathrm{~T}$. The nitride compound of $\mathrm{Sm}$ is found to be semiconducting in its ground state. ${ }^{143}$

Non magnetic $\mathrm{Eu}^{3+}$ ions with six electrons in $4 f$ orbitals have a spin angular momentum $(\mathrm{S})=3$, and an orbital angular momentum $(\mathrm{L})=3$ and therefore $\mathrm{J}=0$. However the spin and orbital angular momenta, both, are quite large separately therefore there is a possibility of a magnetic ordering. ${ }^{148}$ In the case of EuN (and also for $\mathrm{YbN}$ ) different band structures $^{2}$ result for the divalent and the trivalent states of $\mathrm{Eu}(\mathrm{Yb})$. The divalent solution signals half metallic EuN with almost completely filled $f$ levels of the majority spins with opposite magnetic moments on the majority spin N $2 p$ bands which are above the Fermi level. But in the trivalent case, the majority spin $f$ levels exhibit prominent 
hybridization with $\mathrm{N} 2 p$ bands giving rise to a metallic solution. A recent $\mathrm{X}$-ray magnetic circular dichroism (XMCD) study ${ }^{92}$ attempted to solve the question of whether a Eu ion in EuN is divalent or trivalent. The $\mathrm{M}_{4,5}$ and $\mathrm{L}_{2,3}$ edge XMCD absorption spectra show that the origin of the main signal is $\mathrm{Eu}^{3+}$ which is well separated from a weak $\mathrm{Eu}^{2+}$ signals associated with nitrogen vacancies. A van Vleck signature and Brillouin paramagnetic signals are also observed for tri - and divalent EuN. A detailed comparison of spectroscopic data with advanced electronic structure calculations undertook ${ }^{3}$ to settle another outstanding question about the metallicity of EuN. X - ray absorption and emission spectra of epitaxial EuN films grown with activated nitrogen, on comparison with different theoretical models, implied that EuN is a narrow gap semiconductor.

On the theoretical side, an application of a density functional correlated band theory gives a semimetallic picture for the rock - salt structure compound of EuN. This model ${ }^{148}$ predicts considerable polarization of the localized $4 f^{6}$ shell in the ferromagnetic state of EuN. As Eu $5 d$ and N $2 p$ bands are polarized in the opposite directions, the majority $2 p$ bands lie above the minority bands while the majority Eu $5 d$ band overlaps the majority $2 p$ band at the Fermi level creating a gap between respective minority bands. Therefore, within conventional LDA $+U$ band theory, ferromagnetic EuN appears as a half metal. This theory also indicates the atomic nature of the $4 f$ bands, which do not overlap even with neighbouring $4 f$ orbitals. Therefore the direct exchange can be disregarded, however extended Eu $5 d$ states provide an explanation of the localized spins on the Eu ions. ${ }^{148}$

A SIC+LSD treatment predicts a half metallic ${ }^{111,149}$ nature for EuN. Majority $\operatorname{spin} f$ bands in the conduction band are lowered and lie close to $\mathrm{E}_{F}$ and the density of states at the Fermi energy is dominated by $p-f$ bands. There is no $p-f$ hybridization in the minority bands however, and therefore completely filled minority $p$ bands form the valence band and the conduction band consists of completely empty $s-d$ bands, opening up an energy gap of around $1.45 \mathrm{eV}$ in the minority spin channel. ${ }^{111}$ Similar treatments show that europium chalcogenides (EuO, EuSe, EuS and EuTe) are ferromagnetic semiconductors with different band gaps for different spin channels. ${ }^{114}$

Dysprosium with two more $4 f$ electrons than the half filled, has been shown to be a 
ferromagnetic semiconductor ${ }^{112}$ with a $\mathrm{T}_{C}$ value of $25 \mathrm{~K}$. DyN was characterized by the presence of the short - range ferromagnetic correlations at the temperatures far below its magnetic ordering temperature ${ }^{107}$ An extremely high magnetic field, of the order $25 \mathrm{kOe}$, is required to yield a long range ferromagnetic order in DyN. Nitrides of Tb, Ho and Er are also found to favour this arrangement. ${ }^{107}$ There are reports for DyN to possess ferromagnetic order with Curie temperature in the range of $17 \mathrm{~K}$ to $26 \mathrm{~K}^{80,81,150,151}$ A theoretical study, on the other hand, presents an antiferromagnetic picture with a $T_{N}=15 \mathrm{~K} .{ }^{68}$ It seems that an interesting debate on the magnetism of DyN is not yet over.

\subsection{This Work}

Optical studies yield valuable information on the structure of electronic energy bands, making optical spectrometers important tools to study the electronic structure of semiconductors. ${ }^{152-158}$ The above discussion indicates that the most of the effort was exhausted on magnetic and electronic characterization of rare earth nitrides whereas their optical properties comparatively received very little attention. This work intends to fill that void.

Optical properties of the rare earth metals (REM) in paramagnetic and magnetically ordered phase have been studied thoroughly. ${ }^{159-161}$ In - situ reflectance studies of the bcc metal $\mathrm{Eu}$ and the fcc metal $\mathrm{Yb}$ in the range $1.0-11.6 \mathrm{eV}$ does not indicate any optical transition which could be attributed to the $4 f$ electrons ${ }^{162}$ but in contrast $\mathrm{X}$ - ray photoemission measurements on the evaporated thin films of $\mathrm{Eu}, \mathrm{Gd}$ and $\mathrm{Yb}$ provide unambiguous evidence of the $4 f$ transitions. According to this study $4 f$ levels are located $2.1 \mathrm{eV}$ below the Fermi energy for Eu. Similarly for Gd, $4 f$ levels are $7.7 \mathrm{eV}$ below the Fermi energy. A spin - orbit splitting is also resolved for $\mathrm{Yb} 4 f$ levels at $1.4 \mathrm{eV}$ and $2.7 \mathrm{eV}$ below the Fermi level. At room temperature, the optical conductivity $\sigma(\omega)$ and the real part of dielectric function $\varepsilon(\omega)$ are found to be identical for all rare earths. ${ }^{163}$ The optical conductivity at low energy is high due to the free carrier absorption with a broad peak between $1.5 \mathrm{eV}$ and $2 \mathrm{eV}$ indicates a large number of direct optical transitions.

Below the ordering temperatures, new structures in $\sigma(\omega)$ are observed indicating that 
the exchange splitting is dominant at the Fermi level. The relativistic energy bands calculations ${ }^{164}$ predict that the exchange splitting causes the reoccupation of the electronic levels in the energy range within the exchange splitting of the Fermi level. ${ }^{163}$ The spin flip transitions arise due to such reoccupation i.e. transitions between the occupied spin up sub - bands and spin down sub - bands if the spin - orbit interaction is comparable with the exchange splitting energy. The spin - orbit interaction becomes dominant over the exchange splitting from Gd towards Tm. The exchange splitting energies for Ho and Dy are determined to be $0.35 \mathrm{eV}$ and $0.44 \mathrm{eV}$.

There are some decades old results of the optical energy gap measurements of some rare earth nitride compounds. ${ }^{77,83,86,87,110,122,165}$ However it currently is recognized that the procedures used to grow these compounds were vulnerable to oxygen contamination so the results of those reports are compromised. On the other hand current experimental studies on the optical electronic structure ${ }^{1,97-99,166}$ of rare earth nitrides are in sharp contrast to the theoretical reports. ${ }^{2,114,116,118,120,167,168}$

The objective of the present experimental work is to uncover the fundamental optical features of rare earth nitrides by performing reflection/transmission measurements. It aims to settle the outstanding questions on the optical energy gap, index of refraction and dielectric function of rare earth nitride materials. By performing optical measurements at room temperature as well as at a temperature as low as $5 \mathrm{~K}$, this work intends to provide sound footings to the already existing extensive theoretical predictions on the ground state nature of the rare earth nitride compounds. By making additional structural, magnetic and electronic measurements, on the stable, pure, stoichiometric and good quality thin films of rare earth nitrides, this work also intends to remove discrepancies in the experimental works found in literature.

This thesis comprises two sections and eight chapters. Section I is of background and consists of three chapters, the present one being the first.

Optical processes and the properties of general electrodynamic systems are discussed in the second chapter. This chapter highlights the relationship of optical properties with interband electronic transitions, joint density of states and the band structure. The 
determination of optical constants from stratified layers is also discussed in this chapter.

In this primarily experimental work, a variety of experimental techniques have been employed. Growth of rare earth nitride thin films and their optical, magnetic and structural characterization techniques are discussed in chapter 3. Working principles of the equipment are also described briefly in this chapter.

Section II presents the experimental results. Optical properties of four kinds of rare earth nitrides, GdN, DyN, SmN and EuN have been examined in this section. Room temperature and low temperature optical response of GdN is discussed in chapters 4 and 5 respectively. Chapter 6 is about the optical properties of DyN at $300 \mathrm{~K}$ while the last section of this chapter briefly discusses the low temperature response of DyN. The optical properties of both $\mathrm{SmN}$ and $\mathrm{EuN}$ are discussed in chapter 7. A brief literature review about a specific rare earth nitride compound precedes experimental results in each chapter. Each chapter will end with a quick recap of the results.

The final chapter summarises the major results of this thesis and concludes the discussion by shedding light on the future possibilities and challenges. 


\section{Chapter 2}

\section{Optical Properties and Processes in}

\section{General Electrodynamic Physical Systems}

In general, electronic bands are formed once outer orbitals of the atoms overlap with one another in a densely packed solid. The distinct energy levels of a free atom, then, broaden due the formation of chemical bonds which hold the crystal together. However band formation, specifically, happens for every atomic orbital, tightly bound ones giving rise to very atomic - like bands showing very little or no overlap. Electrons stock up the bands to the Fermi energy, $E_{F}$, which is determined by the total electron density. It is the position of Fermi level that determines the metallicity of a compound. An appreciable (negligible) density of states at the Fermi level result in a metal (semimetal). In distinction, half metals have a fully spin polarized electron density of states at the Fermi level. For semiconductors, the Fermi level lies within the energy gap, $\mathrm{E}_{g}$, between the completely filled valence and completely empty conduction bands. It then requires a minimum amount of energy to accelerate electrons to the available states in semiconductors (insulators). An energy gap is the precise question looming over the metallicity of the rare earth nitrides.

The purpose of this chapter is to offer a brief overview of the optical properties of general electrodynamic systems. By confining ourselves to interband electronic transitions, in first section 2.1, a relationship between electronic band structure and optical properties of the crystals will be presented.

Optical constants and the dielectric function are the quantities directly linked to the electronic band structure of a material. However these are not directly discernible by the experiments. Instead these are often extracted through the experimentally evident quantities such as reflectance and transmittance. Section 2.3 of this chapter shows this relation.

Deduction of the complex refractive index from reflection/transmission spectra of a 
multiple layered sample is an intricate problem. Interaction of a light beam with a stratified thin film is generally outlined in the third section.

Salient features of the computer software that was used to analyse the optical spectra are presented in the last section 2.4 .

\subsection{Interband Transitions and Optical Properties}

An optical transition from valence to conduction band is possible if an electron with an energy $\mathrm{E}_{i}$ in the valence band absorbs a photon of energy $\hbar \omega$. According to Pauli's exclusion principle, that final state in the upper conduction must be available therefore by the law of conservation of energy ${ }^{169}$ :

$$
E_{f}=E_{i}+\hbar \omega
$$

where $\mathrm{E}_{f}$ is the energy of electron in its final state. It is apparent from Eq. 2.1 that the minimum value of energy for an interband transition to occur is $\left(\mathrm{E}_{f}-\mathrm{E}_{i}\right)=\mathrm{E}_{g}$.

An energy band gap, $\mathrm{E}_{g}$, can either be direct or indirect. An electronic transition is direct if the valence band maximum and the conduction band minimum, both, occur at the same value of $k$. In an indirect transition, the conduction band minimum occurs at some other value of $k$ which is usually at the zone edge or close.

The interband absorption rate depends on the band structure of a solid. The optical absorption coefficient $\alpha$ is related to the quantum mechanical transition rate $W_{i \rightarrow f}$ for an electron excited from its initial quantum mechanical state $\psi_{i}$ to final state $\psi_{f}$. Therefore in order to determine the frequency dependent $\alpha$, the transition rate, $W_{i \rightarrow f}$ is to be determined first.

The transition rate is given by the Fermi's golden rule: 


$$
W_{i \rightarrow f}=\frac{2 \pi}{\hbar}|M|^{2} g(\hbar \omega)
$$

The first term on the right hand side of Eq. 2.2 is the momentum matrix element between the valence and conduction band states. The momentum matrix element is a function of the electron wavevector and also of the polarization direction of the electric field. The second term is the density of the final states. The transition rate thus depends on these two factors.

External perturbation to the crystal states caused by a radiation field (light wave) is described by the matrix element and is given by ${ }^{170}$ :

$$
M=<\Psi^{\prime}|H| \Psi_{0}>=\frac{e}{m c} \delta_{s_{i}, s_{f}}<\psi_{c k_{f}}|\mathbf{A} \cdot \mathbf{p}| \psi_{v k_{i}}>
$$

where $s_{i}$ and $s_{f}$ are spin indices and $\mathrm{H}$ is the perturbation associated with the light. The vector potential A for the light of frequency $\omega$ can be written as:

$$
\mathbf{A}(\mathbf{r}, t)=A_{0} e^{i(\mathbf{q} \cdot r-\omega t)}+c . c .
$$

where $\mathbf{e}$ is the polarization vector in the direction of the electric field and $\mathbf{q}$ is the wave vector of the radiation.

If $\mathbf{k}_{i}$ and $\mathbf{k}_{f}$, the wave vectors of the initial and final states, are confined to the first Brillouin zone and by neglecting the radiation propagation vector $\mathbf{q}$ (as wave functions vary slowly with $\mathbf{k}$ compared to $\mathbf{q}$ where variation is rapid) then Eq. 2.2 can be simplified to:

$$
W_{i \rightarrow f}=\frac{2 \pi}{\hbar}\left(\frac{e A_{0}}{m c}\right)^{2}\left|\mathbf{e} . \mathbf{M}_{c v}(\mathbf{k})\right|^{2} \delta\left(E_{c}(\mathbf{k})-E_{v}(\mathbf{k})-\hbar \omega\right),
$$

Finally the number of transitions per unit time per unit volume is obtained by summing 
over $\mathbf{k}$, the spin variable $s$ and occupied $(v)$ and empty band $(c)$ band indices. Allowed $\mathbf{k}$ vectors are distributed in the Brillouin zone with a density $V /(2 \pi)^{3}$ where $V$ is the crystal volume:

$$
W(\omega)=\frac{2 \pi}{\hbar}\left(\frac{e A_{0}}{m c}\right)^{2} \Sigma_{c, v} \int_{B Z} \frac{2 d k}{(2 \pi)^{3}}\left|\mathbf{e} \cdot \mathbf{M}_{c v}(\mathbf{k})\right|^{2} \delta\left(E_{c}(\mathbf{k})-E_{v}(\mathbf{k})-\hbar \omega\right)
$$

where the integral extends over the first Brillouin zone and the factor of 2 accommodates electrons of opposite spins.

\subsection{Complex Refractive Index and Dielectric Function}

In the Lorentz oscillator model, real and imaginary parts of a dielectric function are given by ${ }^{171}$ :

$$
\varepsilon^{\prime}=1+\frac{4 \pi N e^{2}}{m} \frac{\left(\omega_{0}^{2}-\omega^{2}\right)}{\left(\omega_{0}^{2}-\omega^{2}\right)^{2}+\Gamma^{2} \omega^{2}}
$$

and

$$
\varepsilon^{\prime \prime}=\frac{4 \pi N e^{2}}{m} \frac{\Gamma \omega}{\left(\omega_{0}^{2}-\omega^{2}\right)^{2}+\Gamma^{2} \omega^{2}} .
$$

In the case of a Drude Oscillator:

$$
\begin{gathered}
\varepsilon^{\prime}=1-\frac{\omega_{p}^{2} \tau^{2}}{\left(1+\omega^{2} \tau^{2}\right)} \\
\varepsilon^{\prime \prime}=\frac{\omega_{p}^{2} \tau}{\omega\left(1+\omega^{2} \tau^{2}\right)}
\end{gathered}
$$

where $\omega_{p}^{2}=4 \pi N e^{2} / m$ and $\gamma=\tau^{-1}$ are plasma frequency and relaxation time, respectively.

The complex dielectric function is related to refractive index $n$ and extinction coefficient 
$k$ as:

$$
\varepsilon^{\prime}=n^{2}-k^{2}
$$

and

$$
\varepsilon^{\prime \prime}=2 n k,
$$

where $\varepsilon^{\prime}$ and $\varepsilon^{\prime \prime}$ are real and imaginary parts of the complex dielectric function. Further, $\varepsilon^{\prime \prime}$ is related to optical conductivity $\sigma$ as following:

$$
\varepsilon^{\prime \prime}=\frac{4 \pi \sigma}{\omega}
$$

When a beam of light traverses through an absorbing medium, then the fractional decrease in intensity of light with distance is given by the relation ${ }^{171}$ :

$$
\alpha=-\left(\frac{1}{I} \frac{d I}{d r}\right)
$$

where $\mathrm{I}$ is the intensity of the light beam and $\alpha$ is absorption coefficient. This can be integrated to obtain:

$$
I(r)=I_{0} e^{(-\alpha r)}
$$

The absorption coefficient $\alpha$ is related to the wavelength $\lambda$ by:

$$
\alpha=\frac{2 \omega k}{c}=\frac{4 \pi k}{\lambda}
$$

Therefore the absorption coefficient is related to the complex refractive index $\tilde{n}$ by:

$$
\tilde{n}(\omega)=n(\omega)+i k(\omega)
$$

where $n$ is the real part of the complex refractive index and $k$ is known as the extinction coefficient. 


\subsubsection{Relation of Transition Rate with Absorption Coefficient}

Combining equations 2.12 and 2.16, we get:

$$
\alpha=\frac{\omega}{n c} \varepsilon^{\prime \prime}
$$

It is known that light in a medium propagates with a velocity $c / n$. Therefore Eq. 2.6, now, gives us a microscopic expression for the absorption coefficient and the other optical constants. By definition the absorption coefficient is energy absorbed per unit time in unit volume divided by the energy flux ${ }^{170}$ :

$$
\alpha=\frac{\hbar \omega W(\omega)}{u(c / n)}
$$

where $\hbar \omega W(\omega)$ is the energy absorbed per unit volume and time, $u$ is the average energy density in a medium of a radiation field and the product of the energy density by the velocity of propagation in the medium, $u(c / n)$, is the energy flux. The average energy density $u$ is described by the vector potential (Eq. 2.4) and is related to the optical constants through the relation:

$$
u=\frac{n^{2} A_{0}^{2} \omega^{2}}{2 \pi c^{2}}
$$

Combining 2.6, 2.18, 2.19 and 2.20, we obtain ${ }^{170}$ :

$$
\varepsilon^{\prime \prime}(\omega)=\frac{4 \pi^{2} e^{2}}{m^{2} \omega^{2}} \Sigma_{v, c} \int_{B Z} \frac{2 d k}{(2 \pi)^{3}}\left|\mathbf{e} \cdot \mathbf{M}_{c v}(\mathbf{k})\right|^{2} \delta\left(E_{c}(\mathbf{k})-E_{v}(\mathbf{k})-\hbar \omega\right) .
$$

This is the basic expression which connects the band structure with the optical properties. Note that it does not depend on the real part of the refractive index. If the matrix element is considered constant then we find that the contribution to the dielectric function from a pair of valence and conduction bands is proportional to $1 / \omega^{2}$ and to the quantity: 


$$
J_{c v}(\hbar \omega)=\int_{B Z} \frac{2 d k}{(2 \pi)^{3}} \delta\left(E_{c}(\mathbf{k})-E_{v}(\mathbf{k})-\hbar \omega\right)
$$

$J_{c v}(\hbar \omega)$ is called the joint density of states because it gives the density of pair of states, filled valence and empty conduction separated by an energy gap $\hbar \omega$.

In three dimensions Eq. 2.22 can be expressed as:

$$
J_{c v}(E)=\frac{2}{(2 \pi)^{3}} \int_{E_{c(\mathbf{k})}-E_{v(\mathbf{k})}=E} \frac{d S}{\mid \nabla\left[E_{c}(\mathbf{k})-E_{v}(\mathbf{k})\right]}
$$

where $d S$ represents an element of surface in $\mathbf{k}$ space.

The real part of the complex dielectric function, $\epsilon^{\prime}(\omega)$, can be obtained by using KramersKronig relation,

$$
\varepsilon^{\prime}(\omega)=1+\frac{2}{\pi} P \int_{0}^{\infty} \omega^{\prime} \epsilon_{2}\left(\omega^{\prime}\right) \frac{1}{\omega^{2}-\omega^{2}} d \omega^{\prime}
$$

where $P$ indicates the principal part. By direct substitution of Eq. 2.21 into Eq.2.24, we get,

$$
\varepsilon^{\prime}(\omega)=1+\frac{8 \pi e^{2}}{m^{2}} \int_{B Z} \Sigma_{v, c} \int_{B Z} \frac{2 d \mathbf{k}}{2 \pi^{3}} \frac{\left|\mathbf{e} . \mathbf{M}_{c v}(\mathbf{k})\right|^{2}}{\left[E_{c}(\mathbf{k})-E_{v}(\mathbf{k})\right] / \hbar} \frac{1}{\left[E_{c}(\mathbf{k})-E_{v}(\mathbf{k})\right]^{2} / \hbar^{2}-\omega^{2}}
$$

The relations 2.21 and 2.25 allow in principal the computation of all optical constants once the band structure is known. 


\subsection{Determination of Optical Constants from}

\section{Transmittance and Reflectance Spectra}

The samples reported in this work are in the form of thin films deposited on sapphire substrate with $\mathrm{MgF}_{2}$ capping layer. Therefore, light falling on the samples encounters air/cap, cap/film, film/substrate and substrate/air interfaces. A beam of light incident on a multilayer where refractive index of incident medium and films in a multilayer are different then a part of the incident beam is reflected in the same medium and other part is transmitted. ${ }^{172}$ Such a sub division of light occurs every time when a light ray hits at an interface of media of discontinuous refractive indices. These multiply reflected and transmitted beams interfere with each other and form maxima and minima in reflectance and transmittance spectra. Optical constants can be deduced from these spectra by various methods. ${ }^{173}$

The problem of determining the light reflected and transmitted at a boundary separating two media is dealt with by applying boundary conditions to the solutions of Maxwell's equations. These require that the tangential components of both electric and magnetic vectors be continuous at the boundary. Consider a plane wave incident on the surface of a single non-absorbing film at an incident angle of $\varphi_{0}$ and angle of refraction $\varphi_{1}$ (Figure 2.1). Amplitude of the electric wave vector of the wave approaching the surface is $\mathrm{E}_{0 i}$. The reflected wave is denoted by $\mathrm{E}_{0 r}$ and the transmitted wave by $\mathrm{E}_{0 t}$.

Amplitudes of the transmitted and reflected vectors in terms of incident vectors can be written as:

$$
\begin{gathered}
r_{s}=\frac{E_{0 r}}{E_{0 i}}=\frac{n_{0} \cos \varphi_{0}-n_{1} \cos \varphi_{1}}{n_{0} \cos \phi_{0}+n_{1} \cos \varphi_{1}}, \\
t_{s}=\frac{E_{0 t}}{E_{0 i}}=\frac{2 n_{0} \cos \varphi_{0}}{n_{0} \cos \phi_{0}+n_{1} \cos \varphi_{1}},
\end{gathered}
$$




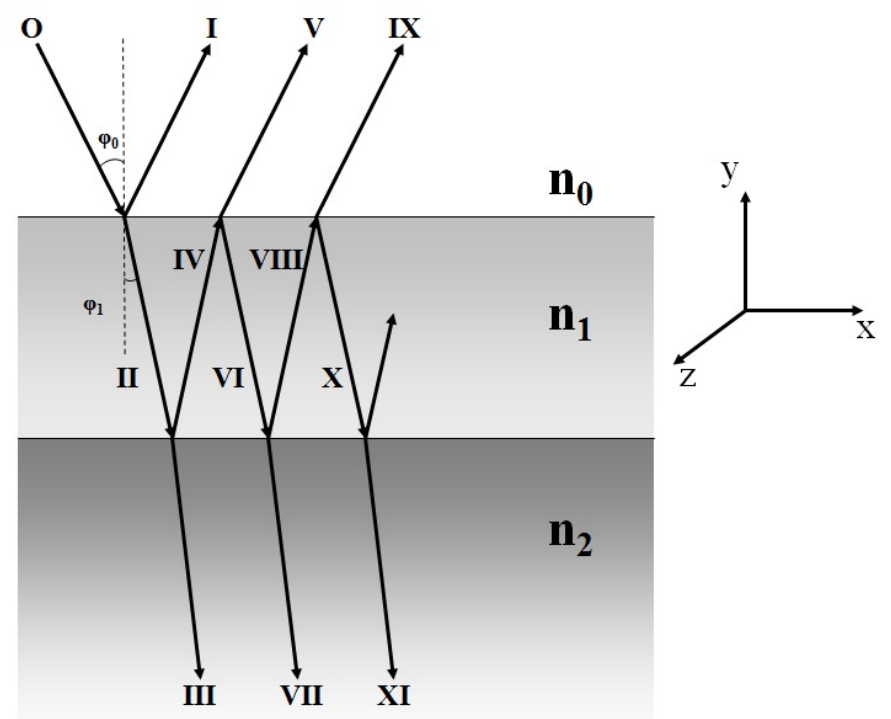

Figure 2.1 Multiple reflection and transmission of light from a single film.

$$
\begin{gathered}
r_{p}=\frac{E_{0 r}}{E_{0 i}}=\frac{n_{0} \cos \varphi_{1}-n_{1} \cos \varphi_{0}}{n_{0} \cos \phi_{1}+n_{1} \cos \varphi_{0}}, \\
t_{p}=\frac{E_{0 t}}{E_{0 i}}=\frac{2 n_{0} \cos \varphi_{0}}{n_{0} \cos \phi_{1}+n_{1} \cos \varphi_{0}},
\end{gathered}
$$

where subscripts $s$ and $p$ represent light with polarization vector lying perpendicular and parallel to the plane of incidence respectively (s-polarized and p-polarized). These equations are called Fresnel coefficients. We may write down the amplitudes of the successively reflected and transmitted beams in terms of Fresnel equations. Note that the values of $r$ and $t$ depend on the direction of propagation of light. So the Fresnel coefficients for the light propagating from medium of refractive index $n_{0}$ to $n_{1}$ are represented by $r$ and $t$. But the corresponding coefficients for the light propagation from $n_{1}$ to $n_{0}$ will be $r^{\prime}$ and $t^{\prime}$. Also from the form of Fresnel reflection coefficients we see that $r^{\prime}=-r$ and $t t^{\prime}=1-r^{2}$. Therefore, in terms of Fresnel coefficients, the amplitudes of the successive reflected beams I, V, IX in Fig. 2.1 are given by $r, t t^{\prime} r^{*},-t t^{\prime} r r^{*^{2}}, \ldots$ and the transmitted amplitudes for the beams III, VII, XI etc. are $t t^{*},-t t^{*} r r^{*}, t t^{*} r^{2} r^{*^{2}}, \ldots$. For the beams II, IV, VI, VII and X, the amplitudes are $t, t r *,-t r^{*} r^{\prime},-t r^{*^{2}} r^{\prime}, t r^{*^{2}}$. When a light beam traverses through the film of refractive index $n_{1}$ and thickness $d_{1}$, a change in phase takes place given by,

$$
\delta=\frac{2 \pi}{\lambda} n_{1} d_{1} \cos \phi_{1}
$$


The reflected amplitudes is thus given by

$$
\begin{gathered}
R=r+t t^{\prime} r^{*} e^{-2 i \delta}-t t^{\prime} r r^{*^{2}} e^{-4 i \delta}+\ldots,=\frac{r+r^{*} e^{-2 i \delta}}{1+r r^{*} e^{-2 i \delta}}, \\
T=t t^{*} e^{-i \delta}-t t^{*} r r^{*} e^{-3 i \delta}+t t^{*} r^{2} r^{*^{2}} e^{-5 i \delta}-\ldots . .=\frac{t t^{*} e^{-i \delta}}{1+r r^{*} e^{-2 i \delta}} .
\end{gathered}
$$

Note that the subscripts $s$ and $p$ are dropped in the above expressions 2.31 and 2.32 because theses are valid for the either direction of polarization provided that $r$ and $t$ are given the appropriate values from equations 2.26, 2.27, 2.28 and 2.29. For non normal incidence, each of the equations 2.31 and 2.32 takes two possible forms. For the light polarized with its electric vector perpendicular to the plane of incidence the reflected and the transmitted amplitudes are obtained by substituting for $r, r^{*}, t, t^{*}$ from the expressions 2.26, 2.27. For the light polarized with the electric vector parallel to the plane of incidence, the Fresnel coefficients are given by 2.28 and 2.29. The reflectance and transmittance, defined as ratios of the reflected and transmitted energy to the incident energy, are then given by:

$$
\begin{gathered}
\mathbf{R}=\frac{r^{2}+2 r r^{*} \cos 2 \delta+r^{*^{2}}}{1+2 r r^{*} \cos 2 \delta+r^{2} r^{*^{2}},} \\
\mathbf{T}=\frac{n_{2}}{n_{0}} \cdot \frac{t^{2} t^{*^{2}}}{1+2 r r^{*} \cos 2 \delta+r^{2} r^{*^{2}}} .
\end{gathered}
$$

For the case of normal incidence, Fresnel equations reduce to:

$$
\begin{aligned}
r & =\frac{n_{0}-n_{1}}{n_{0}+n_{1}}, \\
t & =\frac{2 n_{0}}{n_{0}+n_{1}}, \\
r^{*} & =\frac{n_{1}-n_{2}}{n_{1}+n_{2}}, \\
t^{*} & =\frac{2 n_{1}}{n_{1}+n_{2}} .
\end{aligned}
$$

Thus the reflectance and the transmittance are:

$$
\mathbf{R}=\frac{\left(n_{0}^{2}+n_{1}^{2}\right)\left(n_{1}^{2}+n_{2}^{2}\right)-4 n_{0} n_{1}^{2}+\left(n_{0}^{2}-n_{1}^{2}\right)\left({ }_{1}^{2}-n_{2}^{2}\right) \cos 2 \delta}{\left(n_{0}^{2}+n_{1}^{2}\right)\left(n_{1}^{2}+n_{2}^{2}\right)+4 n_{0} n_{1}^{2}+\left(n_{0}^{2}-n_{1}^{2}\right)\left({ }_{1}^{2}-n_{2}^{2}\right) \cos 2 \delta}
$$




$$
\mathbf{T}=\frac{8 n_{0} n_{1}^{2} n_{2}}{\left(n_{0}^{2}+n_{1}^{2}\right)\left(n_{1}^{2}+n_{2}^{2}\right)+4 n_{0} n_{1}^{2}+\left(n_{0}^{2}-n_{1}^{2}\right)\left({ }_{1}^{2}-n_{2}^{2}\right) \cos 2 \delta}
$$

If a film is absorbing or bounded by absorbing media then values of $n_{0}, n_{1}$ and $n_{2}$ need to be replaced by the complex $\tilde{n}(\omega)=n(\omega)+i k(\omega)$. Resulting expression then become cumbersome and difficult to evaluate. Therefore suitable computer programmes are required.

\subsubsection{Matrix Method Using Fresnel Coefficients}

Degree of complexity increases further when double or more layers are formed. A.W. Crook ${ }^{174}$ has expressed reflected and transmitted amplitudes in terms of Fresnel coefficients. In the presence of absorbing layers this formalism becomes even more cumbersome. However by the application of matrix method ${ }^{172}$, explicit single expressions for the reflectance and transmittance are possible to write. This method is based on Abele's matrix method where forward and backward propagating electric fields are denoted as $E^{+}$and $E^{-}$(Fig. 2.2). Electric vectors for "n" number of layers can also be expressed in terms of the Fresnel coefficients as following:

$$
\left(\begin{array}{c}
E_{m-1}^{+} \\
E_{m-1}^{-}
\end{array}\right)=\frac{1}{t_{m}}\left(\begin{array}{cc}
e^{i \delta_{m-1}} & r_{m} e^{i \delta_{m-1}} \\
r_{m} e^{-i \delta_{m-1}} & e^{-i \delta m-1}
\end{array}\right)\left(\begin{array}{c}
E_{m}^{+} \\
E_{m}^{-}
\end{array}\right)
$$

Here $r_{m}$ and $t_{m}$ are Fresnel coefficients for the reflection and transmission from $m$ th layer. For a system of $n$ number of layers, we require to know the relation between $E_{n+1}^{+}$and $E_{0}^{+}$so that the transmission coefficient may be obtained; and also that between $E_{0}^{-}$and $E_{0}^{+}$for the reflection coefficient. From Eq. 2.41 we obtain,

$$
\left(\begin{array}{c}
E_{0}^{+} \\
E_{0}^{-}
\end{array}\right)=\frac{\left(C_{1}\right)\left(C_{2}\right) \ldots\left(C_{n+1}\right)}{t_{1} t_{2} \ldots t_{n+1}}\left(\begin{array}{c}
E_{n+1}^{+} \\
E_{n+1}^{-}
\end{array}\right),
$$

where 


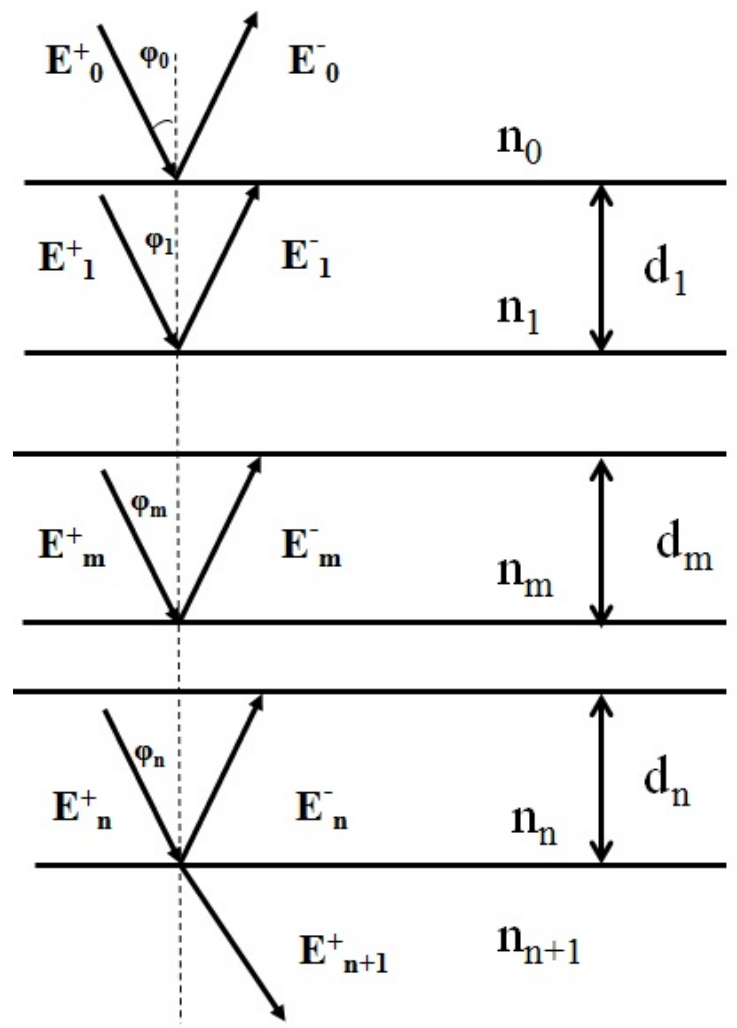

Figure 2.2 A multilayer sample.

$$
\left(C_{m}\right)=\left(\begin{array}{cc}
e^{i \delta_{m-1}} & r_{m} e^{i \delta_{m-1}} \\
r_{m} e^{-i \delta_{m-1}} & e^{-i \delta m-1}
\end{array}\right)
$$

We may therefore express $E_{0}^{-}$and $E_{n+1}^{+}$in terms of $E_{0}^{+}$and so obtain the reflection and transmission coefficients. We note, however, that since there is no negative-going wave in the $(n+1)^{t h}$ medium, we put $E_{n+1}^{-}=0$. Writing the matrix product

$$
\left(C_{1}\right)\left(C_{2}\right) \ldots\left(C_{n+1}\right)=\left(\begin{array}{ll}
a & b \\
c & d
\end{array}\right)
$$

we obtain from Eq. 2.42

$$
R=\frac{E_{0}^{-}}{E_{0}^{+}}=\frac{c}{a},
$$




$$
T=\frac{E_{n+1}^{+}}{E_{0}^{+}}=\frac{t_{1} t_{2} \ldots t_{n+1}}{a} .
$$

The reflectance and transmittance, therefore, are given by

$$
\mathbf{R}=\frac{\left(E_{0}^{-}\right)\left(E_{0}^{-}\right)^{*}}{\left(E_{0}^{+}\right)\left(E_{0}^{+}\right)^{*}}
$$

and

$$
\mathbf{T}=\frac{n_{n+1}}{n_{0}} \frac{\left(E_{n+1}^{+}\right)\left(E_{n+1}^{+}\right)^{*}}{\left(E_{0}^{+}\right)\left(E_{0}^{+}\right)^{*}}
$$

so that we obtain

$$
\begin{gathered}
\mathbf{R}=\frac{c c^{*}}{a a^{*}}, \\
\mathbf{T}=\frac{\left(t_{1} t_{2} \ldots t_{n+1}\right)\left(t_{1}^{*} t_{2}^{*} \ldots t_{n-1}^{*}\right)}{a a^{*}} .
\end{gathered}
$$

For a non absorbing single film, above expressions return the same values of reflectance and transmittance as calculated in Eq. 2.33 and Eq. 2.34. 


\subsection{TFCalc Software}

The situation was more involved for the case of our samples, where a protecting layer of $\mathrm{MgF}_{2}$ was deposited on rare earth nitride thin films to prevent oxidation, compared to the discussion above. Thus, an incident beam of light encounters four interfaces in all, air-cap, cap-film, film-substrate and substrate-air when it is transmitted. All these media have discontinuous refractive indices therefore one expects the subdivision of light into transmitted and reflected beams at every interface. Further, these grown films are definitely not ideal and interfaces are strictly not parallel. Also all these layers have variable thickness, capping layers usually were $30 \mathrm{~nm}$ to $150 \mathrm{~nm}$ thick while REN films were from $250 \mathrm{~nm}$ to $775 \mathrm{~nm}$ thick therefore multiply reflected and transmitted beams when interfere, form an irregular pattern. In order to extract the optical constants, a commercial software ${ }^{175}$ was used which makes use of the characteristic matrix method as described in previous section. The software, TFCalc ${ }^{175}$, allows the user to specify the environment of the coating layers along with incident and exit medium and wavelength range. Experimental reflectance and transmittance data can be entered in the form of text files. Analysis of the experimental spectra is then done in steps. First, the subgap region is fitted by assuming $k=0$ and certain value of refractive index. Once a reasonable fit is produced, the above gap region is produced by keeping the refractive index constant. Finally, the frequency dependence of refractive index is allowed and low energy ranges are also fitted for the free carriers. 


\section{Chapter 3}

\section{Experimental Techniques}

Though the optical properties of rare earth nitrides are the main focus of the present work, several other experimental techniques were also employed at different stages according to the requirements of the project. As it happens in any experimental thesis, a complete familiarity with the apparatus; its structure and the working principles are of monumental importance in order to conduct a good experiment and to obtain meaningful data. A description of all those experimental procedures and methods, as demanded by this project, is the central point of this chapter.

The first task was to prepare good quality thin films of rare earth nitrides. Section 3.1 discusses the details of thin film growth in ultra high vacuum (UHV) environment.

Soon I knew the craft of experimental
physics was beyond me - it was the
sublime quality of patience - patience
in accumulating data, patience with
recalcitrant equipment - which I sadly
lacked.
Dr. Abdul Salam
(Physics Nobel Laureate 1979)

Section 3.3 is about the electronic and magnetic measurements. Magnetic measurements were performed by a superconducting quantum interference device (SQUID). Although the magnetic data is of utmost significance in itself, in this work it primarily is used for the identification purpose.

The last three sections, 3.4, 3.5 and 3.6 are devoted to the Fourier Transform Infra Red spectrometer and the conventional UV/Vis spectrophotometer, used to conduct the optical transmittance and reflectance measurements. 


\subsection{Growth of Rare Earth Nitride Thin Films}

Growth of rare-earth nitride thin films was particularly a challenging task. Due to the propensity of rare earth metals to react with the atmosphere, ultra high vacuum environment was required for the growth of their nitride compounds. Also, a protecting layer was required for these thin films in order to prevent oxidation and debris once they are exposed to air. The other issues to address were the correct stoichiometry of REN thin films and their ability to withstand several room temperature and low temperature measurements which were made over the course of many weeks.

\subsubsection{Preparations for the Growth}

Gadolinium (Gd), Dysprosium (Dy), Samarium (Sm) and Europium (Eu) (99.9\% pure) were bought from Kurt J. Lesker Company in the form of $3 \mathrm{~mm}-6 \mathrm{~mm}$ size pieces. Ingots of Gd, Sm \& Eu were stored in mineral oil therefore they were cleaned with either ethanol or isopropanol before putting them in the growth chamber. Dy, Sm \& Eu were thermally evaporated whereas an electron gun was used to evaporate Gd. For thermal evaporation, rare earth metals were placed in tantalum (Ta) or tungsten (W) boats because of their extremely high temperature at the vapour pressure of $10^{-4}$ Torr. Some of the physical properties ${ }^{176}$ of RE metals and boat materials are summarized in Table 3.1.

Table 3.1 Physical Properties of Rare Earth Metals and Boat Materials

\begin{tabular}{|c|c|c|c|}
\hline Metal & $\begin{array}{c}\text { Density } \\
\mathrm{g} / \mathrm{cm}^{3}\end{array}$ & $\begin{array}{c}\text { Melting Point } \\
{ }^{\circ} \mathrm{C}\end{array}$ & $\begin{array}{c}\text { Temp. for vap. press. } 10^{-4} \text { torr } \\
{ }^{\circ} \mathrm{C}\end{array}$ \\
\hline $\mathrm{Dy}$ & 8.6 & 1409 & 900 \\
\hline $\mathrm{Eu}$ & 5.2 & 1380 & 950 \\
\hline $\mathrm{Gd}$ & 7.96 & 1312 & 1175 \\
\hline $\mathrm{Sm}$ & 7.5 & 1072 & 573 \\
\hline $\mathrm{MgF}_{2}$ & 3.2 & 1266 & 1540 \\
\hline $\mathrm{Ta}$ & 16.6 & 2996 & 2590 \\
\hline $\mathrm{W}$ & 19.3 & 3410 & 2757 \\
\hline $\mathrm{Ga}$ & 5.9 & 30 & 907 \\
\hline $\mathrm{Al}$ & 2.7 & 660 & 1010 \\
\hline
\end{tabular}


For the protecting layers, $\mathrm{MgF}_{2}(99.8 \%$ pure powder), $\mathrm{AlN}$ or GaN were the materials of choice. They are transparent in the energy range of our interest, $0.5 \mathrm{eV}$ to $5.0 \mathrm{eV}$. We found that while the $\mathrm{MgF}_{2}$ was excellent to keep the REN thin films safe from contamination for days and easy to deposit, its insulating properties made it difficult to measure electrical properties of the REN thin films. Therefore some of the REN films were grown with GaN films on top.

The choice of the substrate is usually dictated by the measurements needed. In our case, wafers of double sided polished sapphire $\left(\mathrm{Al}_{2} \mathrm{O}_{3}\right)$ were used. Sapphire wafers, cplane (0001), were $50.8 \mathrm{~mm}$ in diameter. Prior to mounting on the sample holders, silver (Ag) contacts were also grown on the substrates in order to measure in-situ resistance of the grown REN films. Carbon tape was used to hold the substrates on the sample holder. Special care was taken to clean the boats, RE metal ingots, substrate holder and sapphire wafers before putting them in the growth chamber.

\subsubsection{Structure of UHV Growth Chamber}

A UHV chamber that was used to grow thin films of REN contained electrodes to hold boats, electron gun, ionization source, sample holder and a quartz crystal used as thickness monitor as shown in Fig. 3.1. The electrodes connected to the contact pads on the substrates led to a multimeter outside the chamber to monitor in-situ resistance measurement of the film. A variable power supply was used to control the flow of current through the boats. A continuous flow of water cooled the electrodes. Pieces of RE metals were placed in Ta boats whereas a $\mathrm{W}$ boat held $\mathrm{MgF}_{2}$ powder. The inside of the UHV chamber was vacuum cleaned before shutting it down. An ultra high vacuum was produced inside the chamber with the help of a backing pump and a turbo pump. The inside pressure of the growth chamber was lowered to around $10^{-2} \mathrm{mbar}$, first, by the backing pump. Later the turbo pump, which was capable of achieving a pressure as low as $10^{-8}$ mbar or even better, was switched on. 


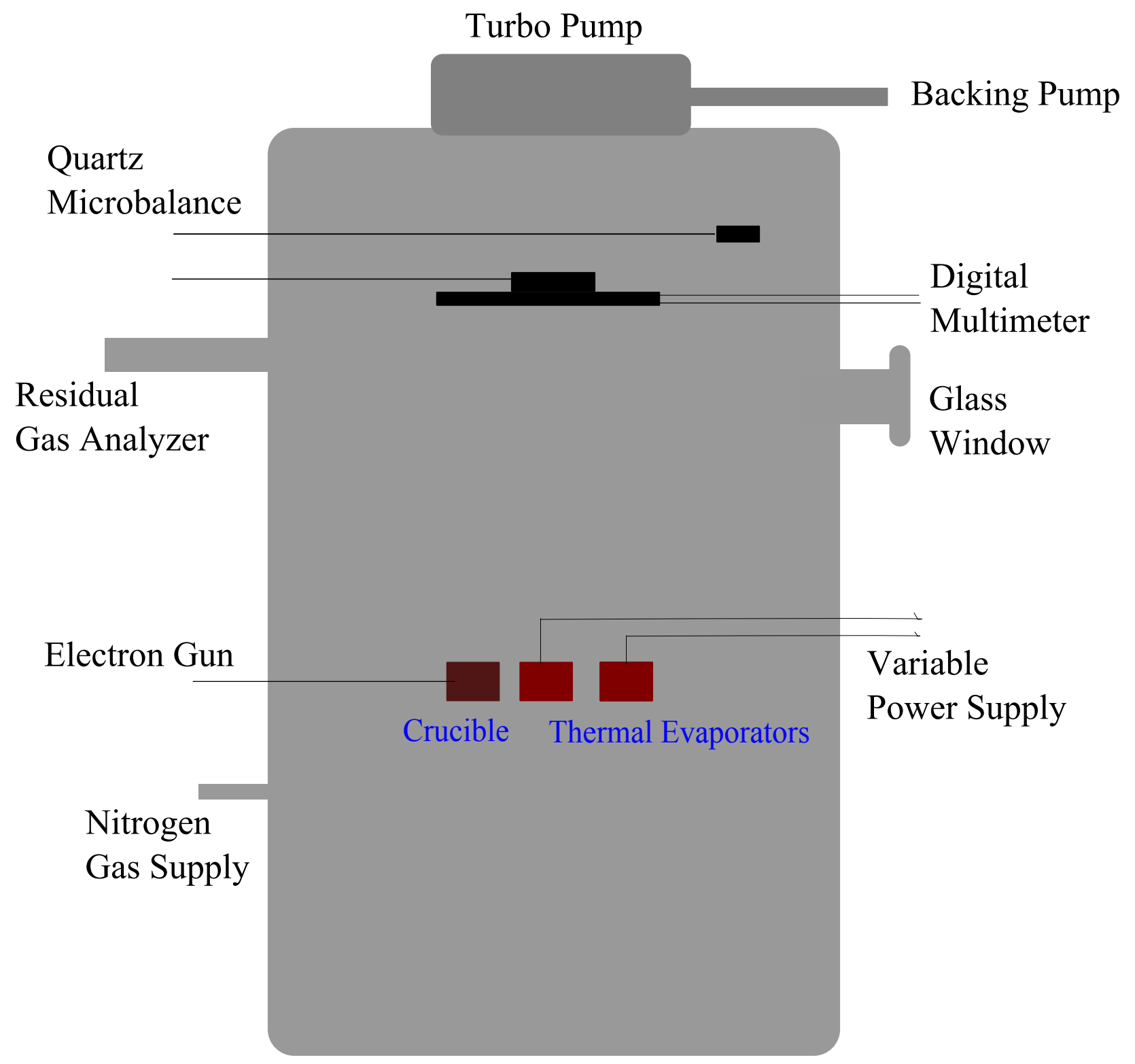

Figure 3.1 Schematic diagram for a UHV growth chamber. 


\subsubsection{Growth Procedure}

To achieve a high level of purity in our thin films, the growth chamber was heated thoroughly for almost a week. The outside of the growth chamber was covered with the aluminium foil and then the silicone extruded heating tapes were wrapped around all parts of the chamber followed by another layer of the aluminium foil. According to the manufacturer's specifications, duo-tape heating cables of width $2.5 \mathrm{~cm}$ and $240 \mathrm{~cm}$ in length were designed to achieve a baking temperature of more than $200^{\circ} \mathrm{C}$ with a maximum power of 416 watts. The temperature of the heating tapes was raised slowly by a gradual increase of current through them by a variable power supply. Whenever the temperature of the cables was increased, an increase in the pressure was noticed which, after few hours, would fall away. And then the current through the cables was further increased. This way, contaminants inside the chamber, which were mainly composed of $\mathrm{H}_{2}, \mathrm{He}, \mathrm{Ar}, \mathrm{O}_{2}$ and water molecules were evacuated. The composition of residual gases was monitored by a mass spectrometer attached to the growth chamber through a valve. Moreover, outgassing of the growth chamber was done while the chamber was still being baked. During this process, the RE metal and the capping layer materials were slowly evaporated for approximately 10 minutes. Remaining $\mathrm{O}_{2}$ molecules or $\mathrm{H}_{2} \mathrm{O}$ molecules would stick to the walls of the chamber due to the gettering effects of the RE metals to reduce the pressure further. Ultra high purity $\mathrm{N}_{2}$ gas was also passed through a Ni sponge scrubber into the chamber. This careful procedure of the outgassing would significantly reduce $\mathrm{O}_{2}$ pressure, our major contamination concern. A simple calculation by using the kinetic theory of gases would enable us to determine the number of $\mathrm{O}_{2}$ molecules present inside the chamber.

In a typical solid, the area taken by an atom in a monolayer corresponds to the interatomic distance i.e. $(0.3 \mathrm{~nm})^{2} \approx 0.1 \mathrm{~nm}^{2}$. So to form a monolayer, the number of atoms per unit area is of the order $10^{19} \mathrm{~m}^{-2}$. Every molecule that bounces from a surface imposes an impulse of $2 m v_{x}$ where $m$ is the mass of the molecule and $v_{x}$ is the velocity of the particle in the $x$-direction. The pressure exerted by each molecule on the walls of the chamber is given by: 


$$
P=\frac{F}{A}
$$

where the force $F$ is defined as the rate of change of momentum, $\Delta m v_{x} / \Delta t$ where $m$ is the mass of the molecule. A molecule will travel a distance of $2 L$ in time $t$ so the Eq. 3.1 will become

$$
P=\frac{2 m v_{x} / 2 L}{A}=\frac{2 m v_{x}^{2}}{2 L^{3}}
$$

Considering that there are $N$ molecules present per unit volume moving in the $x$-direction with a velocity $v_{x}$ and $m=\frac{M}{N_{A}}$ where $M$ is molar mass of the particle and $N_{A}$ is the Avogadro constant equal to $6.02214129(27) \times 10^{23} \mathrm{~mol}^{-1}$ then the Eq. 3.2 takes the form,

$$
P=\frac{2 M N v_{x}^{2}}{2 N_{A}}
$$

Thus number of molecules hitting a surface per unit time per unit area, to know when we get a monolayer of atoms assuming that every one sticks, is given by:

$$
\frac{N v_{x}}{2}=\frac{P N_{A}}{2 M v_{x}}
$$

To calculate the velocity $v^{2}=v_{x}^{2}+v_{y}^{2}+v_{z}^{2}$, we use

$$
v_{r m s}=\sqrt{\frac{3 R T}{M}},
$$

where $R$ is the ideal gas constant and $T$ is temperature with values of $8.314 \mathrm{~J} / \mathrm{mol} . \mathrm{K}$ and $300 \mathrm{~K}$, respectively. For the oxygen molecules of molar mass $M=0.032 \mathrm{Kg}, v_{r m s}$ is approximately $491 \mathrm{~m} / \mathrm{s}$. Therefore, for $\mathrm{O}_{2}$ partial pressure of the order $10^{-8}$ Torr $=$ $4.2 \times 10^{-7} \mathrm{~Pa}$, number of oxygen molecules per unit area per unit time inside the chamber would be around:

$$
\frac{N v}{2}=\frac{4.2 \times 10^{-7} \times 6 \times 10^{23}}{2 \times 0.032 \times 491}=8.1 \times 10^{15} \mathrm{~m}^{-2} \mathrm{~s}^{-1}
$$


Thus, the flux of oxygen molecules under these conditions is $8.1 \times 10^{15}$ molecules $\mathrm{m}^{-2} \mathrm{~s}^{-1}$. Our calculations thus show that oxygen contamination in our samples is less than $1 \%$.

After the outgassing, the heating was switched off and the growth chamber was allowed to cool down to the room temperature for a day. A base pressure of the order $10^{-9}$ mbar was achieved after the outgasing. To grow a REN thin film, high purity $\mathrm{N}_{2}$ gas was allowed to enter the growth chamber, raising the chamber pressure to around $10^{-4}$ mbar. The flow of $\mathrm{N}_{2}$ gas was controlled by a mass flow controller at a rate of $3 \mathrm{sccm}$ to $4 \mathrm{sccm}$. For the thermal evaporation, the current of the electrodes carrying boats of RE metal was gradually increased. When a pressure of $10^{-4}$ or $10^{-5}$ mbar, the so called growth pressure, became stable, the shutter was opened to expose the substrate to the evaporated RE metals. The growth rate $(\AA / s)$ and thickness of the film were monitored from a thin film thickness and rate controller. During thermal evaporation, the growth rate always fluctuated and therefore the average growth will be quoted in this work. After achieving the required thickness of a REN film, it was quickly capped with a $\mathrm{MgF}_{2}$ layer, which also was evaporated thermally. Samples were stored in a vacuum chamber and whenever required to do measurements, were transported in a desiccator.

Preparation of GdN thin films involved an electron gun and a deposition rate controller (DRC). The deposition rate controller was controlled by a sequencing mode. This is discussed in detail in chapter 4.

A number of films were prepared with different growth parameters and thickness. The description of all the samples of a particular REN thin film are tabulated in the respective chapter of each material in the Results \& Discussions section of this thesis. Growth of all rare earth nitride thin films took place at room temperature in the UHV room, School of Chemical and Physical Sciences, Victoria University of Wellington. 


\subsection{XRD and RBS}

X-ray diffraction (XRD) and Rutherford back scattering (RBS) techniques were used to for the structural characterization of the REN thin films.

The X-ray diffraction is a versatile technique that is useful in not only identification but also to determine the grain size, preferred orientation and lattice spacing etc. ${ }^{177,178}$ When a monochromatic X-ray beam irradiates the sample, the atomic lattice of the sample acts as a three dimensional diffraction grating. This causes the X-ray beam to be diffracted at the specific angles. The diffraction pattern obtained includes the position (angles) and the intensities of the diffracted beam and carries information about the sample. The interplanar spacings (d-spacing) are calculated from the angles of the diffracted beam. The position of the diffracted peaks also provides information about how the atoms are arranged within the sample (unit cell size or lattice parameters and crystal structure).

When certain requirements are met, scattered X-rays interfere constructively to produce the diffraction pattern. This condition, known as Bragg's law, is given by:

$$
n \lambda=2 d \sin \theta
$$

where $d$ is the interatomic spacing, measured in $\AA, \theta$ is the angle of diffraction measured by the diffractometer as $2 \theta$ and $\lambda$ is the wavelength of incident radiation. Peaks in the diffraction pattern were compared with the tabulated data to identify the crystal structure. HighScore Plus software supplied with the PANlytical X-ray diffractometer was used to analyse XRD data of REN thin films. The width of the peaks in a diffraction pattern is an indication of crystallite size which can be calculated using the Debye-Scherrer equation:

$$
D=\frac{0.9 \lambda}{\beta_{1 / 2} \cos \theta}
$$

where $\beta_{1 / 2}$ is full width at half maximum.

The Rutherford back scattering technique is also a powerful tool for the materials 
analysis. ${ }^{179,180}$ Although it is widely used in near the surface analysis, in our project, it was used to determine the composition of atoms in the thin films of rare earth nitride compounds. In this technique a target is bombarded with ions of the energy typically between $0.5-4 \mathrm{MeV}$. Ions, usually ${ }^{4} \mathrm{He}^{+}$, will suffer elastic collision with the atoms of the sample and backscatter. The energy of backscattered ions, which is dependent on the mass of the atom with which they have interacted and the distance travelled inside the material, carries information about the depth profile and the relative compositional weights of the elements present inside the film. It is a quantitative technique without the need for reference sample. This method is sensitive to the elements with the masses larger than the incident particle. For lighter mass elements, or in the case where the material is thick enough that backscattered particles from different elements overlap in the RBS spectrum, other techniques such as Elastic Recoil Detection or Nuclear Reaction Analysis (NRA) may be necessary.

A well-established library of the scattering cross-sections and the recoil energies for the elements of different masses exists, and using this, a simulated RBS spectrum can be calculated from a model of multiple layers comprised of given compositions and thickness. In practice, this model is refined until the simulated spectrum most closely matches the experimental spectrum. In this study the RBS simulation program RUMP was used to find the desired values.

A 3MV Van-de-Graaff accelerator at the National Isotope Centre, GNS Science was used to do RBS measurements. The measurements were performed and the data were analysed by Dr. John Kennedy and Dr. Peter Murmu. 


\subsection{Temperature dependent resistivity and}

\section{Magnetometry.}

Temperature dependent resistivity measurements were performed in order to study electrical properties of rare earth nitrides. The measurements were done by using the van der Pauw technique. ${ }^{181}$ This technique is popular to determine the resistivity of semiconducting materials.
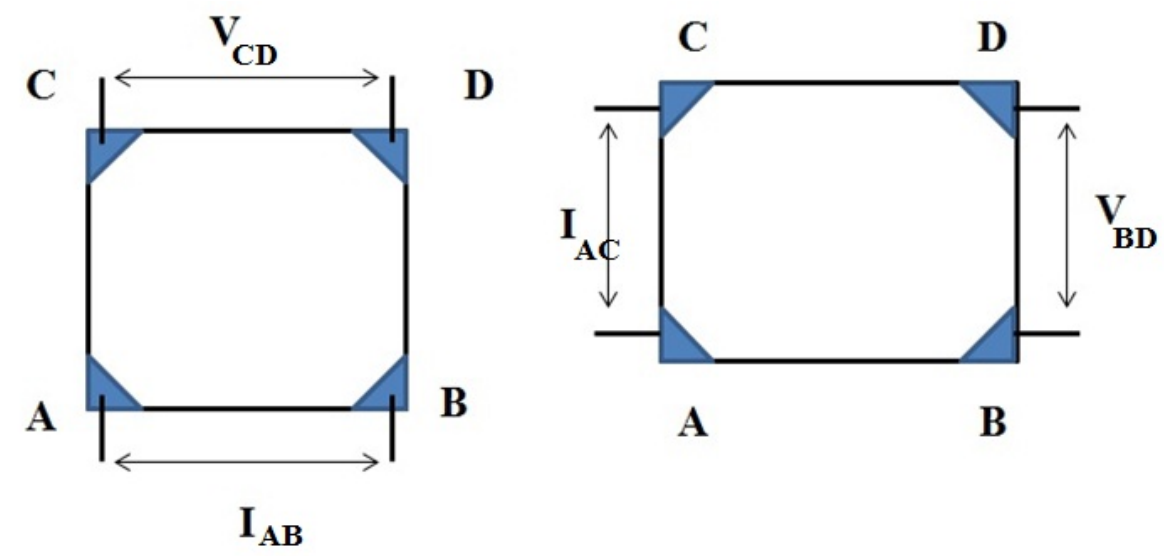

Figure 3.2 A schematic of a rectangular van der Pauw configuration.

REN thin films were fabricated on a square sapphire substrate with four silver contact pads grown on each of its corners as shown in Fig. 3.2. In a suitable geometry, the diameter of the contact pads must be smaller than the distance between the contacts. Two characteristic resistances, say $\mathbf{R}_{A}$ and $\mathbf{R}_{B}$, were measured which are related to the sheet resistance via following equation:

$$
e^{-\pi R_{A} / R_{S}}+e^{-\pi R_{B} / R_{S}}=1
$$

where $R_{A}=V_{C D} / I_{A B}$ and $R_{B}=V_{B D} / I_{A C} . R_{S}$ is the sheet resistance given by $R_{S}=$ $\pi R / \ln 2$. The sheet resistance is a measure of resistance of thin films that are nominally uniform in thickness. The resistivity, $\rho$, is calculated by $\rho=R_{s} d$, where $d$ is the film thickness.

Magnetic properties were measured by a superconducting quantum interference device. As the name implies, the device consists of superconducting coils to produce a magnetic field. A sample, usually of area $15 \mathrm{~mm}^{2}$, is placed inside a plastic straw and mounted in 
the sample holder. The sample holder moves the sample in an applied DC magnetic field and a flux change is produced in the superconducting coils. The change in flux is detected by changes in the voltage across the Josephson junction which is major part of a SQUID.

To observe the magnetic behaviour, the sample was first cooled without the presence of a magnetic field and then in its presence, referred to as zero field cooled and field cooled measurements respectively. Hysteresis curves were also obtained.

Magnetic measurements were performed at Industrial Research Limited, Gracefield, by Dr. Simon Granville and Dr. Shen Chong. Temperature dependent resistivity measurements were performed by Tanmay Maity and Binh Do Le at the School of Chemical and Physical Sciences, Victoria university of Wellington.

\subsection{Optical Measurements}

The optical reflectance and the transmittance $(\mathrm{R}, \mathrm{T})$ measurements were made in two steps. In the energy range of $0.5 \mathrm{eV}$ to $2.5 \mathrm{eV}$, measurements were made by a Fourier Transformation Infra Red (FTIR) Spectrometer. A conventional UV/Vis spectrophotometer was used to make R, T measurements from $1.0 \mathrm{eV}$ to $5.0 \mathrm{eV}$. Results of both the experiments were stitched together to produce the full spectrum.

\subsubsection{Optical Measurements in the Infrared Region}

A FTIR spectrometer, BOMEM model DA8, was used to make reflectance and transmittance measurements in the infrared region. Table 3.2 lists the frequency ranges which were covered by a combination of beam splitters, detectors and filters in a typical reflectance/transmittance measurements by FTIR spectrometer. 


\subsubsection{Working Principle}

Fourier transform infrared spectroscopy is essentially based on a conventional Michelson interferometer. Details of theory and general introduction on the principles of operation of this technique can be found in several texts. ${ }^{182,183}$ In this technique, an infrared spectrum is obtained first by collecting an interferrogram of a sample signal (Fig. 3.3). A Fourier transform of this signal is then performed, to obtain the spectrum (Fig. 3.4). An inteferometer consists of a beam splitter, a fixed mirror and a movable mirror which moves forwards and backwards. A beam splitter divides an incident ray into transmitted and reflected ray. The transmitted ray travels to the fixed mirror and reflected ray goes to moving mirror. Upon reflection from these mirrors they recombine again at the beam splitter. Since an optical path difference (OPD) is introduced between the light rays reflected from fixed mirror and movable mirror, they interfere to make an interferogram. The inteferogram is the plot of light intensity and optical path difference. The large peak, called the centre burst, is due to the zero path difference when all the wavelengths are inphase. But as the optical path difference grows, reflected beams are never fully in-phase again producing the spikes of the lesser intensity.

Table 3.2 Frequency Range of FTIR

\begin{tabular}{|c|c|c|c|c|c|}
\hline Frequency Range & Beam Splitter & Filter & Attenuation & Detector & Gain \\
\hline $\mathrm{cm}^{-1}$ & & & $\%$ & & \\
\hline $2000-8500$ & $\mathrm{CaF}_{2}$ & Mesh & 70 or 90 & $\mathrm{InSb}$ & 1 \\
\hline $4000-14000$ & Quartz & Mesh & 70 or 90 & $\mathrm{InSb}$ & 4 \\
\hline $8500-17000$ & Quartz & Blue & No & $\mathrm{Si}$ & 16 \\
\hline
\end{tabular}




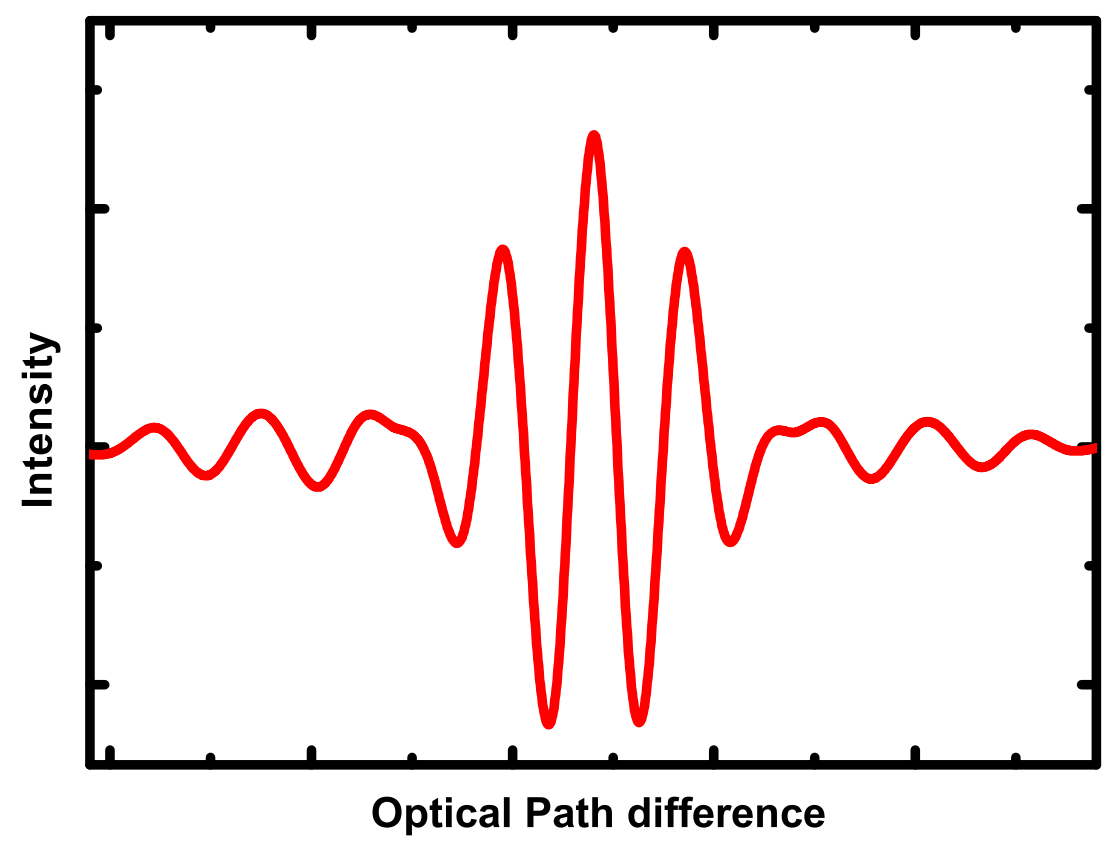

Figure 3.3 An interferogram from a GdN thin film.

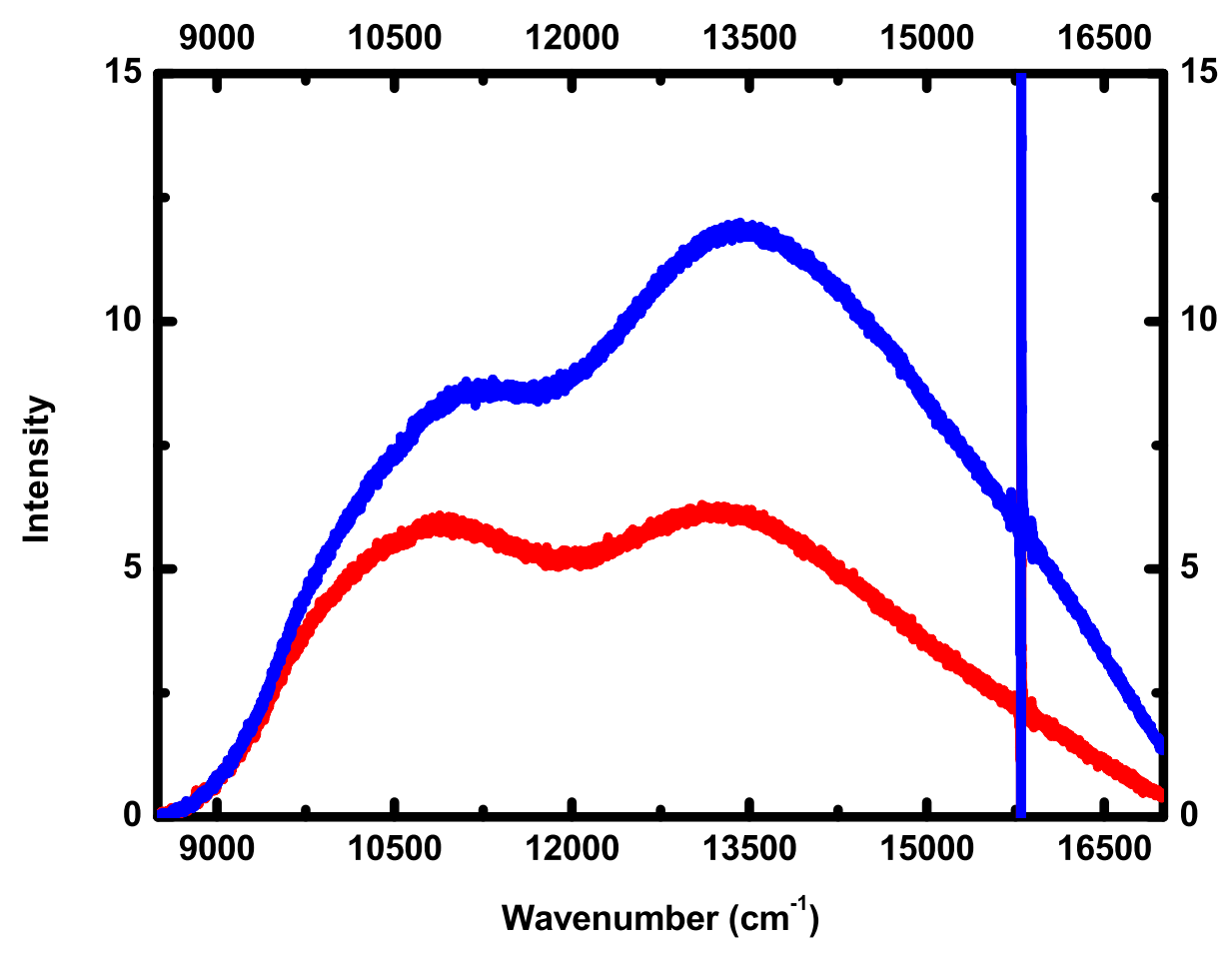

Figure 3.4 Fourier Transform of interferogram signals from a GdN sample (red) and from empty slots of the sample holder (blue) to the frequency domain. 


\subsubsection{Transmission Measurements}

For the transmission measurements at the room temperature, samples were mounted on a rotating sample holder. A transparent sample compartment cover enabled us to see that the correct sample was positioned in front of the beam. Each time, a reference spectrum was obtained from the empty slot of the sample holder before passing the beam through the sample. This procedure was repeated from 10 to 20 times in every frequency range and each time 50 scans were made at a resolution of $2 \mathrm{~cm}^{-1}$. Such a process was required to reduce the measurement uncertainty which was caused by the drift in the source signal. A single measurement would take a little over a minute. In order to minimize the uncertainty due to the signal drift, reference spectra were analysed first. The ratio of the each reference spectrum was taken with the previous one, $\mathbf{R}_{n+1} / \mathbf{R}_{n}$, (Fig. 3.5) Transmittance was calculated with respect to the average of the reference spectra with ratio equal to 1 .

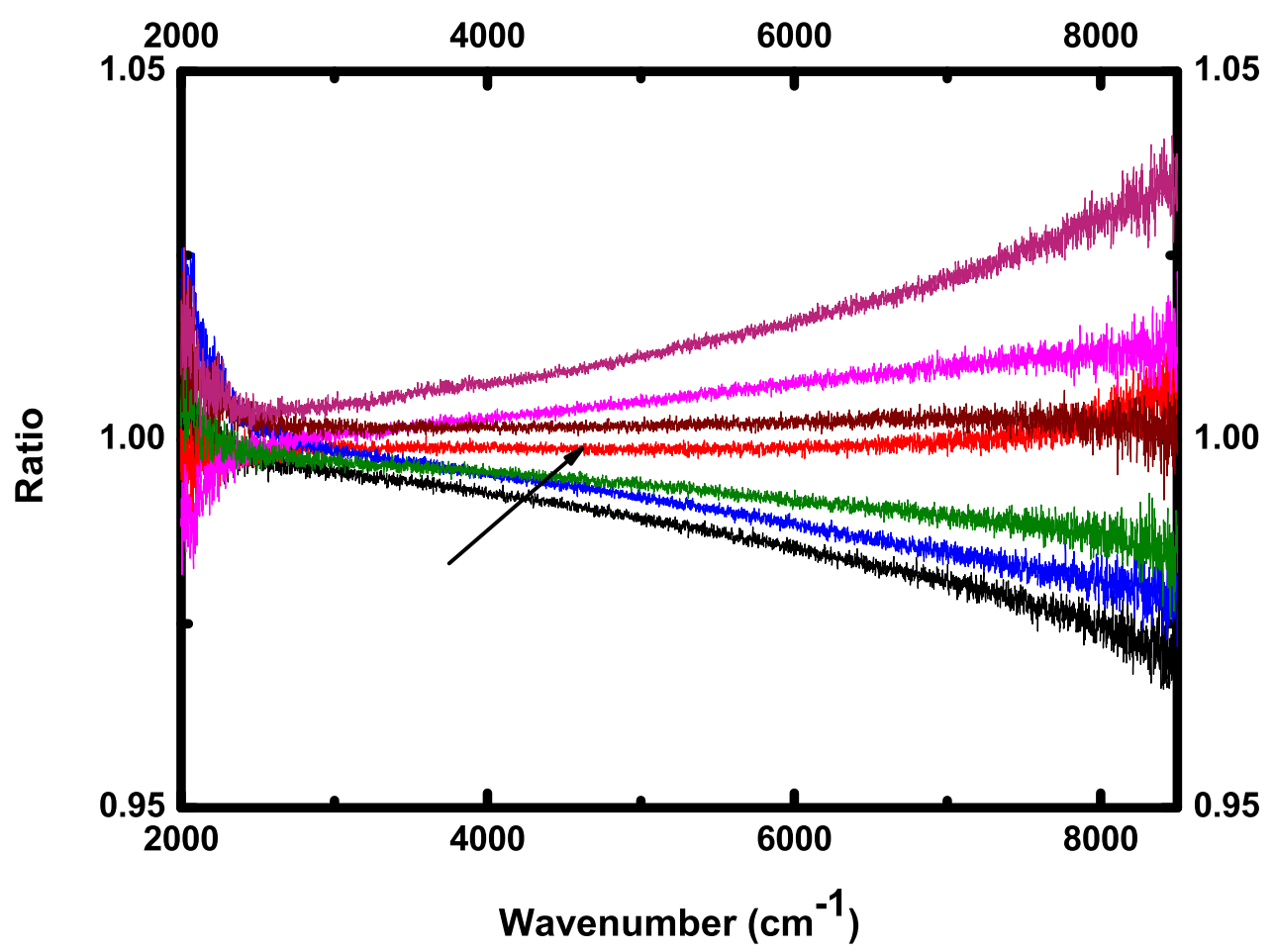

Figure 3.5 Ratios of reference spectra in frequency range $2000 \mathrm{~cm}^{-1}$ to $8500 \mathrm{~cm}^{-1}$. 


\subsubsection{Reflection Measurements}

A reflectance geometry, placed inside the sample compartment, was used to make the reflection measurements at room temperature. The sample was mounted on a sample holder, along with a gold film which was used as a reference standard for the reflectance measurement. Before placing it in the sample compartment, the optical axis of the sample was adjusted to the gold film with the help of three grub screws. A laser was used to do the optical axis adjustment. The sample holder was transferred inside the sample chamber and the optical axis was checked once again to make sure that it was not disturbed during the transfer. The process to collect reflectance spectra and its analysis was essentially the same as for the transmittance measurements.

\subsection{Transmittance Measurements at Low Temperature}

The low temperature transmittance measurements are a significant part of the present project. The objective was to determine a direct energy gap value for RENs in their ferromagnetic phase. For this purpose an optistat CF cryostat was used.

\subsubsection{Operational Setup}

A combination of rotary pump, diffusion pump and ion gauge was used to create a high vacuum in the outer vacuum chamber. The sample space was purged with $\mathrm{N}_{2}$ gas which acted as heat exchanger between the sample and the cryogen which was either liquid nitrogen or liquid helium. As soon as the temperature fell below $77 \mathrm{~K}, \mathrm{~N}_{2}$ gas was purged with He gas as the heat exchanger. The cryogenic fluid was supplied to the cryostat from a dewar through an insulated transfer tube. It would flow through a heat exchanger around the sample space and out of the cryostat to the pump. The flow of the cryogen was controlled either by the valve on the transfer tube or on the pump. The temperature 
was actually controlled with the combination of these valves and proportional - integral - derivative (PID) functions on the temperature controller. To make sure that the transfer tube was not blocked with frost, it was gently heated prior to going inside the transfer arm of the cryostat. The heat exchanger had a thermometer and a heater mounted over it which was controlled by the temperature controller. The sample would not come in contact with the cryogen. It was made sure that the pressure in the sample space was such that the heat exchange gas was not condensed inside the sample space which would interfere with the optical measurements. In the temperature range of $295 \mathrm{~K}$ to $77 \mathrm{~K}$, measurements were taken at $50 \mathrm{~K}$ intervals by using the liquid nitrogen. With liquid $\mathrm{He}$, however, the transmittance spectra were collected at every $5 \mathrm{~K}$ interval down to $5 \mathrm{~K}$. The conditions for the transmittance measurements at low temperature were the same as of room temperature measurements. However, since light would heat the sample, the intensity of the light, for the low temperature measurements, was reduced by using a smaller aperture, $0.5 \mathrm{~mm}$, and a filter which would attenuate $90 \%$ of the light. Each scan was carefully run when the sample temperature was stable enough. A procedure similar to that for the room temperature data was used to analyse the low temperature transmittance data to reduce the uncertainty in measurements.

\subsection{Optical Measurements in UV/Vis Range}

The reflectance and the transmittance measurements in the range of $1.0 \mathrm{eV}$ and $5.0 \mathrm{eV}$ were made by using a conventional UV/Vis spectrophotometer. A tungsten light source was used. Light would pass through a monochromator to a collimator and was then focussed on the sample of REN thin film, mounted on a rotating sample holder. Transmittance and reflectance were recorded via a silicon detector. The position of the silicon detector was different in each configuration. Alignment was made by using a laser of wavelength $543.36 \mathrm{~nm}$. To determine the transmittance, as described in the previous section, the light intensity from the sample was divided by the light intensity through an empty slot. In the case of reflectance, a quartz wedge was used as a reflection standard. The experiment was performed in a dark room and samples were exposed to air during the 
measurements, as opposed to the FTIR spectrometer where samples were in the vacuum. However due to the effective protecting layers of $\mathrm{MgF}_{2}$, physical properties of the REN thin films were not changed in measurement duration.

Optical measurements were made at Industrial Research Limited, Gracefield, under the supervision of Dr. Nick Strickland and Dr. Annette Koo. 


\title{
SECTION 2
}

\author{
RESULTS AND \\ DISCUSSIONS
}




\section{Chapter 4}

\section{Gadolinium Nitride}

$\mathrm{A} \mathrm{Gd}^{3+}$ ion has a half-filled $4 f$ shell with 7 electrons of unpaired spin. Gd is located in the middle of the rare-earth group in the periodic table. ${ }^{184}$ The earliest measurements ${ }^{121,123}$ indicated that $\mathrm{GdN}$ is ferromagnetic with $\mathrm{T}_{c}=69 \mathrm{~K}$ and a magnetic moment of $228 \mathrm{emu} / \mathrm{g}$ $\left(7.0 \mu_{B} / \mathrm{Gd}\right.$ atom) at $4.2 \mathrm{~K}$ if all moments are aligned parallel. ${ }^{121,123,124,129,131,134,185} \mathrm{~A}$ band structure calculation ${ }^{115}$ using the augmented plane wave method (APW) predicted a semiconducting ground state for GdN with a narrow band gap of $0.2 \mathrm{eV}$. It is also suggested that GdN obeys the Curie-Weiss law at temperatures higher than its magnetic transition. Further studies ${ }^{125,126}$ on the stoichiometric polycrystalline GdN with a lattice constant of 4.974 Åhave shown that its Curie temperature is $58 \mathrm{~K}$ instead of $66 \mathrm{~K}$ or $69 \mathrm{~K}$ while the saturation magnetic moment is $6.84 \mu_{B} / \mathrm{Gd}, 2 \%$ less than the theoretical value of $7.0 \mu_{B} / \mathrm{Gd}$ atom.

The debate on the physical properties of GdN was reignited in the last decade because of its potential spintronic applications. ${ }^{18,68,69,111,186}$ In this regard, the first significant study was done by Leuenberger et al. ${ }^{104}$ who reported GdN to show the ferromagnetic ordering below $60 \mathrm{~K}$. A theoretical study by Larson et al. ${ }^{114}$, showed that GdN along with EuO is a ferromagnetic semiconductor. This proposition was further corroborated by Granville et al ${ }^{187,188}$, Ludbrook et al. ${ }^{94}$ and Preston et al. ${ }^{112}$ with reported value of $\mathrm{T}_{c}$ of $68 \mathrm{~K}$. Electronic structure calculations ${ }^{68,114,120,133,140}$ provide varying interpretations to the band structure of GdN. Duan et al. ${ }^{68,133}$ proposed that a next - nearest - neighbour (NNN) antiferromagnetic super exchange mechanism and RKKY - type nearest neighbour exchange coexist in compounds of Gd with nitrogen. More recent reports fuel the need for further investigation. Yoshitomi et al. ${ }^{97-99,166}$ report $\mathrm{T}_{C}$ of $30 \mathrm{~K}$ in $95 \mathrm{~nm}$ and $30 \mathrm{~nm}$ thick films of GdN, the lowest reported yet. Further, Senapati et al. ${ }^{130,132}$ speculated an antiferromagnetic phase is present in $\mathrm{GdN}$, introduced due to the local strain produced by nitrogen vacancies.

While the arguments continue on the magnetic properties, a few groups are now turning 
their attention to the optical properties of GdN. Previously Schneemeyer et al. ${ }^{122}$ reported an energy gap of GdN greater than $1.5 \mathrm{eV}$ through transmittance measurements. It showed a transmittance of less than $20 \%$ in the regions far below the gap. The samples were claimed to be single crystals, though. Theoretical calculations ${ }^{114,116,119}$ came up with contradicting values of $1.1 \mathrm{eV}-1.2 \mathrm{eV}, 0.98 \mathrm{eV}$ and $5.0 \mathrm{eV}$.

The first systematic investigation of the optical energy gap, on reliable samples of GdN, was presented by Trodahl et al. ${ }^{1}$ Figure 4.1 shows the band structure calculated with the value of $U_{d}=8.0 \mathrm{eV}$ determined from the paramagnetic and ferromagnetic gaps reported from their data. The valence band in Fig. 4.1 is composed of $\mathrm{N} 2 p$ levels whereas Gd $5 d$ make the conduction band. Gd $6 s$ states are above $4.0 \mathrm{eV}$. Unoccupied $4 f$ levels are represented by narrow bands above $4.0 \mathrm{eV}$ whereas occupied $4 f$ levels are 7-10 eV below the Fermi level. Red slid lines in Fig. 4.1 represent the majority spin states and the dashed blue lines are the minority one. The dispersion of the bands shown in Fig. 4.1 begins by plotting the energy for increasing $\boldsymbol{k}$ outwards from the origin called $\Gamma=2 \pi / a(0,0,0)$ point to the $\mathrm{X}$ point at $(2 \pi / \mathrm{a})(1,0,0)$. We then move across the $\mathrm{L}$ point at $\pi / a(1,1,1)$ via the $\mathrm{W}$ point, and back to the origin. Finally we go out again from the origin to the $\mathrm{X}$ point via the $\mathrm{K}$ point. The valence band in Fig. 4.1 is comprised of $\mathrm{N} 2 p$ states whereas the conduction band is due to $\mathrm{Gd} 5 d(6 s)$ states.

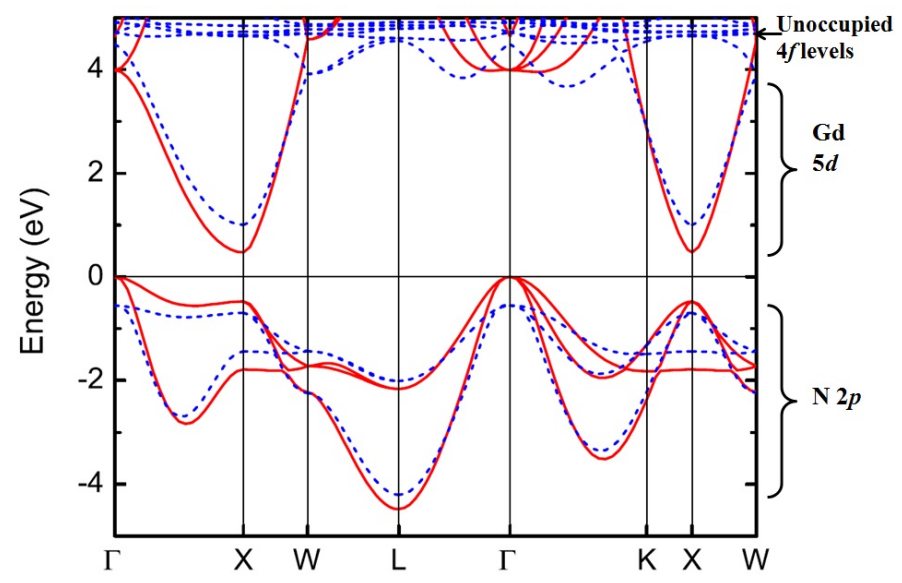

Figure 4.1 The band structure of GdN from Trodahl et al. ${ }^{1}$. The solid lines represent majority - spin bands, and dashed lines minority.

To the best of our knowledge, there is only one report ${ }^{165}$ about the refractive index of GdN. These films were prepared by a plasma-radio frequency sputtering method and were deposited on glass substrates. There are several questions regarding the reported data. 
First of all, it does not state what material was used to protect the films from oxidation. Then, the sputtered films are of amorphous nature and thus show an expanded lattice constant which might well be $\mathrm{Gd}_{2} \mathrm{O}_{3}$. The authors also report a wide optical gap of $4.1 \mathrm{eV}$.

Motivation for the present chapter mainly comes from claims as discussed above which differ greatly in many aspects. Understanding of the optical properties of polycrystalline GdN thin films and the determination of their optical constants will be the main subject. However, we will make our case by discussing various other physical properties studied by different experimental techniques. XRD spectra of representative $\mathrm{GdN}$ thin films will be discussed first followed by an examination of the DC electrical resistivity data. After brief comments on magnetic data, we will focus on the reflectance/transmittance spectra obtained from the polycrystalline GdN thin films. Temperature dependent optical properties of GdN will be discussed in the next chapter.

\subsection{Preparation of GdN Thin Films}

Ingots of $99.9 \%$ pure Gd were evaporated in the presence of pure $\mathrm{N}_{2}$ gas in an ultra high vacuum environment. Due to the extremely high temperature ${ }^{176}\left(1175^{\circ} \mathrm{C}\right)$ (Table 3.1) at which the vapour pressure of $\mathrm{Gd}$ equals $10^{-4}$ torr, an electron gun was used to evaporate Gd metal pieces. The power supply, which provided high voltage to the electron gun, was controlled by the control output voltage of a deposition controller sensor card.

The deposition controller, along with an oscillator and crystal sensor, provide the basis to monitor and control the deposition process manually or automatically. ${ }^{189}$ A quartz crystal microbalance, which was in the UHV chamber but not in close proximity to the substrate (Fig. 3.1) was used as a crystal sensor. A quartz crystal microbalance (QCM) measures a mass per unit area by measuring the change in the frequency of a quartz

crystal resonator. ${ }^{190,191}$ The controller then derives the deposition rate and the thickness of the deposited material from this information when the deposited material density is known. In a sequencing mode (auto mode), a user programmable proportional, integral, 
derivative (PID) loop was used to monitor the deposition process and to control the power supply. The PID control loop automatically adjusts variables to hold the measurement at the set point and essentially eliminates the need for continuous attention to the operation. In our measurements, a programme sequence included deposition rate as a set point while other parameters like the density $\left(\mathrm{g} / \mathrm{cm}^{3}\right)$ of the material to be deposited, the maximum thickness of the film, maximum power limit and time for power ramp and power soak were also entered. During a sequence run, an error which is defined as the difference between the set point and measurement, is responded by the proportional gain term which would reduce the rise time but not the over shoot of the signal. An integral term forces the rate control error to reach the set point by giving the controller a larger gain with the slowest time constant (low frequencies). However it will not control the oscillatory response which will be eliminated by a derivative term which is the rate of change of measurement with time. A typical film deposition cycle is shown in Fig. 4.2. ${ }^{176}$

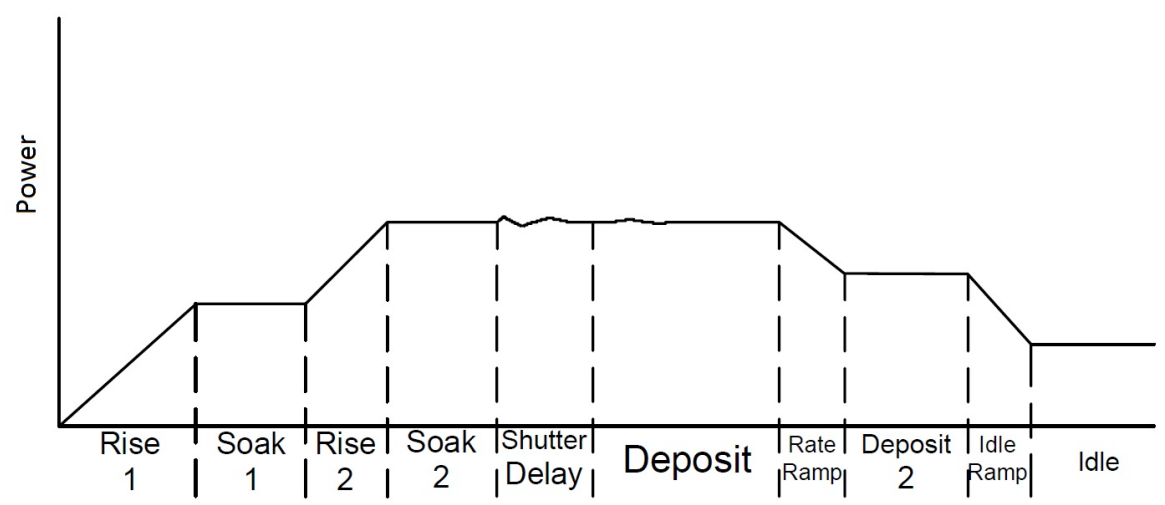

Figure 4.2 Typical film deposition cycle.

Films of $\mathrm{GdN}$ were covered with layers of $\mathrm{MgF}_{2}$ or $\mathrm{GaN}$ to protect them from the atmosphere. All films were grown on c-plane (0001) sapphire wafers. The wafers were $50.8 \mathrm{~mm}$ in diameter and $0.5 \mathrm{~mm}$ thick. During the growth wafers were held at room temperature.

Resistance measurements were made in-situ. Before the film deposition begins, the resistance between the contact pads, grown on the substrate, was infinite. As the film growth begins, resistance between the contacts starts to fall rapidly. After some time, when the film is a few hundred nanometers thick, the resistance of the film becomes almost saturated. Figure 4.3 shows the reciprocal of resistance of the grown film, 
measured in-situ, against the film thickness. Uniform film growth could be inferred from the linear form of the plot. The resistivity, calculated from the slope of this plot, is about $0.45 \pm 0.03 \mathrm{~m} \Omega \mathrm{cm}$. Note that the resistance of the film was measured even during the growth of the capping layer as well as when the sample was being taken out of the chamber to expose it to the air. A change of the resistivity during these processes would be a manifestation of the film being oxidised. However, no or rather slight changes in the resistivity were observed in some cases showing effectiveness of the protecting layers.

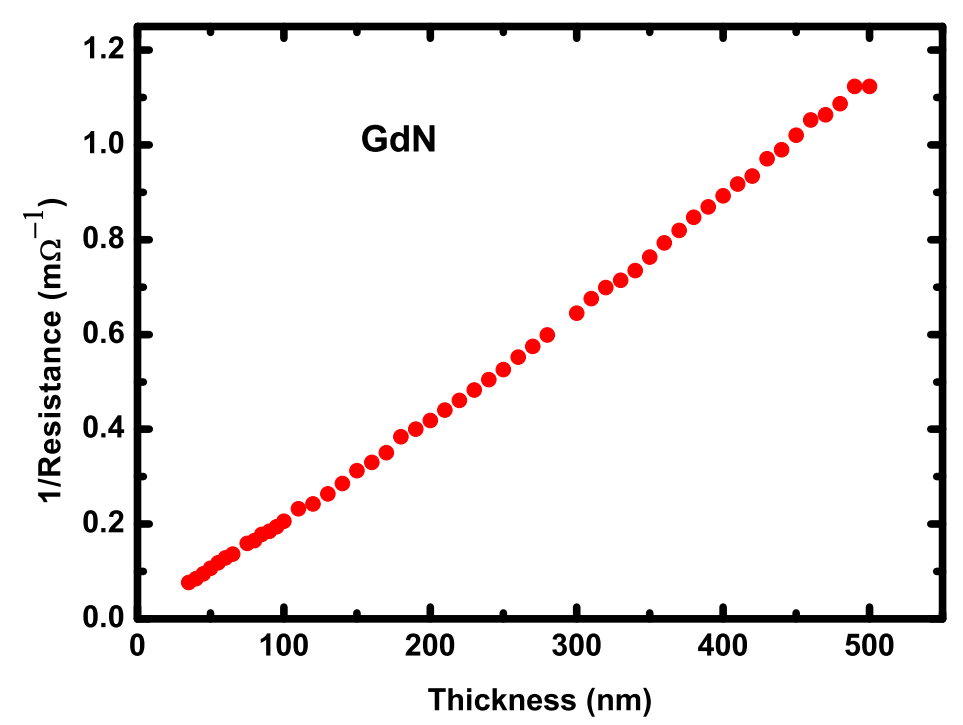

Figure 4.3 Reciprocal of film resistance vs thickness for a GdN sample prepared at room temperature. 
Table 4.1 lists all of the GdN thin films along with their growth parameters. A base pressure lower than $10^{-8}$ mbar was achieved for the growth of all thin films. As described in chapter 3, a low base pressure ensures that the films are relatively free from contaminants such as oxygen and water molecules and that the films are only composed of $\mathrm{Gd}$ and $\mathrm{N}$ atoms.

Table 4.1 Growth Parameters of GdN Thin Films grown at Room Temperature

\begin{tabular}{|c|c|c|c|c|}
\hline $\begin{array}{c}\text { Sample } \\
\text { ID }\end{array}$ & $\begin{array}{c}\text { Growth } \\
\text { Pressure } \\
(\mathrm{mbar})\end{array}$ & $\begin{array}{c}\text { Deposition } \\
\text { Rate } \\
(\AA / \mathrm{A})\end{array}$ & $\begin{array}{c}\text { Film } \\
\text { Thickness } \\
(\mathrm{nm})\end{array}$ & $\begin{array}{c}\mathrm{N}_{2} / \mathrm{Gd} \\
\text { Flux ratio }\end{array}$ \\
\hline \hline G1 & $1.2 \times 10^{-4}$ & 0.5 & 320 & 156 \\
\hline G2 & $2.1 \times 10^{-4}$ & 0.5 & 450 & 273 \\
\hline G3 & $1.5 \times 10^{-4}$ & 1.0 & 420 & 197 \\
\hline G4 & $8.9 \times 10^{-5}$ & 2.0 & 650 & 29 \\
\hline G5 & $1.4 \times 10^{-4}$ & 0.5 & 740 & 181 \\
\hline G6 & $1.4 \times 10^{-4}$ & 0.5 & 775 & 181 \\
\hline
\end{tabular}

The nitrogen pressure inside the growth chamber is represented by the growth pressure while the deposition rate is for Gd atoms deposited on the substrate. A higher nitrogen pressure makes certain that the nitride compounds of RE metals are close to being stoichiometric. In other words the higher ratio of $\mathrm{N}_{2} / \mathrm{Gd}$ atoms per unit area per unit time, more likely is the GdN film will be stoichiometric. Therefore our choice of these growth parameters was mainly dictated by our desire to grow GdN with a 1:1 ratio. To determine the $\mathrm{N}_{2} / \mathrm{Gd}$ flux ratio i.e. the number of nitrogen atoms falling on the substrate to $\mathrm{Gd}$ atoms, we use the ideal gas theory as described in section 3.1.3. By using Eq. 3.4, for the case of GdN (G5) sample, the nitrogen flux is calculated to be around $2.90 \times 10^{20} \mathrm{~m}^{-2} \mathrm{~s}^{-1}$. The number of Gd atoms can be determined from the deposition rate as:

$$
\frac{1}{A} \frac{d N}{d t}=\rho \frac{d z}{d t}
$$

Where $\rho$ is the number of $\mathrm{Gd}$ atoms per unit volume $\approx 3.2 \times 10^{28} \mathrm{~m}^{-3}$ and $z$ is the thickness of the film. The second factor on the R.H.S. of Eq. 4.1 represents the deposition rate. For a deposition rate of $0.5 \AA / \mathrm{s}$, the flux of Gd atoms is thus $1.6 \times 10^{18} \mathrm{~m}^{-2} \mathrm{~s}^{-1}$. Therefore the 
flux ratio of nitrogen to $\mathrm{Gd}$ atoms falling on a substrate is around 181 . Note that not every nitrogen atom falling on the substrate sticks to it. However it is expected that around $1 \%$ of nitrogen atoms hitting the substrate surface stick to it and bond with Gd atoms.

The thickness of the films were verified by RBS measurements. RBS measurements provide us with rare earth atomic concentration in the sample in units of atoms $\mathrm{cm}^{-2}$. Dividing this by the number of rare earth atoms per unit volume, the film thickness can be estimated.

\subsection{Structural Analysis}

Figure 4.4 shows the XRD pattern of a representative $\mathrm{GdN}(\mathrm{G} 4)$ thin film. The most pronounced peak comes from the sapphire substrate. The peaks labelled (111), (200) and (222) are from GdN.

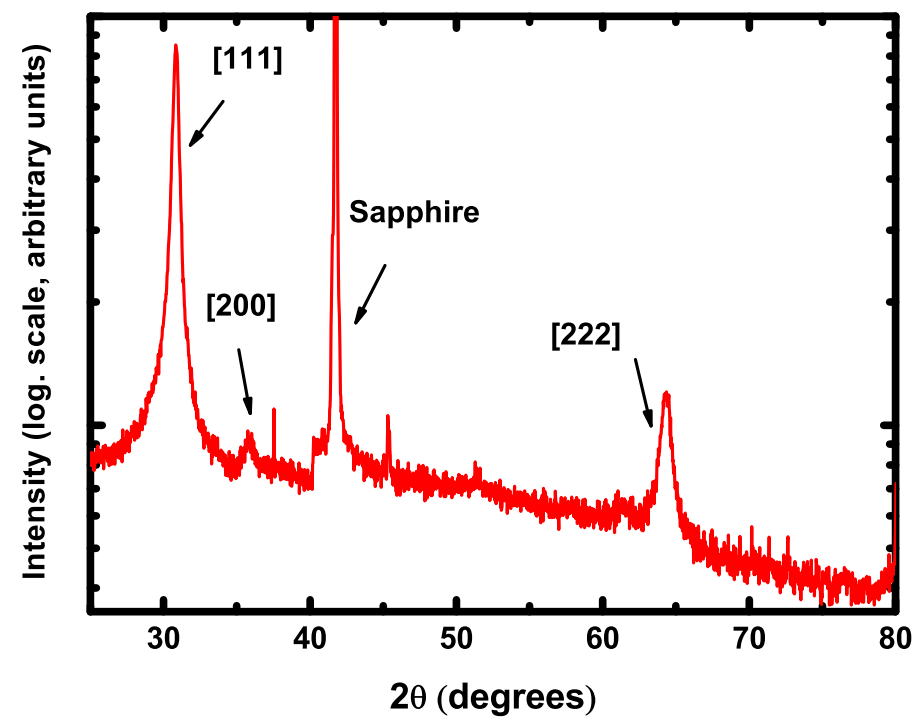

Figure 4.4 XRD pattern of a representative $G d N(G 4)$ thin film.

As indicated by the intensity of the diffracted peaks, the grown film has a preferred orientation along the $<111>$ direction. A slightly expanded lattice constant of average $0.504 \pm 0.003 \mathrm{~nm}$ is calculated, greater than the experimental standard value of bulk GdN which usually is in range $0.497 \mathrm{~nm}-0.499 \mathrm{~nm} \cdot{ }^{73,74,94,125,126}$ Since oxygen contamination 
could decrease the lattice constant ${ }^{124,129}$, it is justifiable to deduce that the larger lattice constant in fact confirms our observation of low oxygen contamination in the present GdN samples.

Note that it has been observed ${ }^{94}$ that at the initial stages, a GdN film is vulnerable to oxidation at the film - YSZ substrate interface. In the present work, however, the substrate is sapphire therefore it is highly unlikely for an oxide layer to form at the film/substrate interface. Also we found later that water molecules inside the UHV chamber were absorbed by $\mathrm{MgF}_{2}$ powder which landed on the film surface forming a insulating layer of $\mathrm{Gd}_{2} \mathrm{O}_{3}$ however $\mathrm{MgF}_{2}$ was carefully out gassed.

The other interesting feature is the crystallite size in the thin films of GdN presented here. The crystallite size in these GdN films is a function of the film thickness. The smallest is $5.7 \mathrm{~nm}$ in a film of thickness around $300 \mathrm{~nm}$ and it nearly doubles in the films of twice the thickness. All films thicker than $600 \mathrm{~nm}$ have grain size of $10 \pm 1 \mathrm{~nm}$. The grain size for polycrystalline thin films being reported here is significantly smaller than that of epitaxial GdN film $(30 \mathrm{~nm})$ prepared by pulsed laser deposition ${ }^{91,94}$ but matches well with $10 \mathrm{~nm}$ reported on polycrystalline films. ${ }^{188}$

\subsection{Temperature Dependent DC Resistivity and Magnetism}

The temperature dependent resistivity of a $\mathrm{GdN}$ thin film from $5 \mathrm{~K}$ to $300 \mathrm{~K}$ is shown in Fig. 4.5. The film was prepared under the same conditions as of G6 sample (Table 4.1) but were capped with GaN. The measurements were made on the GdN thin films capped with GaN while cooling from room temperature to $5 \mathrm{~K}$ (black solid line) and then back to $300 \mathrm{~K}$ (red dotted curve). As illustrated, the resistivity increases gradually from its room temperature value of $0.14 \Omega \mathrm{cm}$. The rise becomes steeper below $100 \mathrm{~K}$ and it reaches its maximum value, $0.5 \Omega \mathrm{cm}$ at $50 \pm 1 \mathrm{~K}$, before falling to $0.3 \Omega \mathrm{cm}$ at $15 \mathrm{~K}$. The room temperature resistivity in our experiment is smaller than the reported values of $0.3 \Omega \mathrm{cm}, 0.5 \Omega \mathrm{cm}$ and $1-1000 \Omega \mathrm{cm}$ which presented a semiconducting model of 
GdN. ${ }^{137,188,192}$ But it is significantly larger than those which proposed a semiconducting to metallic transition model ${ }^{104}$ of $\operatorname{GdN}(10 \mathrm{~m} \Omega \mathrm{cm})$ as well as epitaxial ${ }^{90,94}(0.18 \mathrm{~m} \Omega \mathrm{cm})$ and heteroepitaxial ${ }^{75}$ films prepared on $\mathrm{Si}$ and $\mathrm{GaN}$ substrates respectively $(0.1 \mathrm{~m} \Omega \mathrm{cm})$.

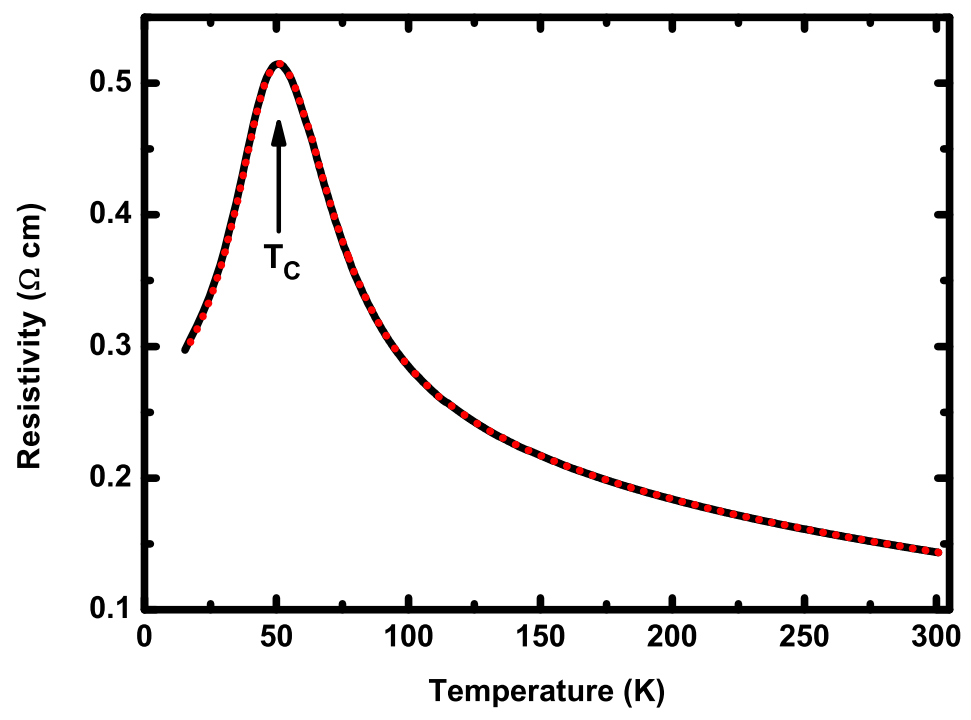

Figure 4.5 Temperature dependence of DC resistivity for GdN. The sample was prepared under the conditions similar to G6 but capped with GaN.

The specific behaviour of the resistivity below $50 \mathrm{~K}$ is of interest. We see that the resistivity at $15 \mathrm{~K}$ is nearly twice as big as the room temperature resistivity. While the present work does not show the data below $15 \mathrm{~K}$, previous reports ${ }^{188}$ show that the resistivity increases by decreasing the temperature further, establishing the ferromagnetic semiconducting ground state of GdN. From the ambient - temperature resistivity, we could estimate a carrier concentration of the order $10^{18} \mathrm{~cm}^{-3}$ by using the equation:

$$
\sigma_{D C}=\frac{N e^{2} \tau}{m^{*}}
$$

where $m^{*} \approx 0.27 m_{0}$ is the electron effective mass determined from the band structure ${ }^{2}$ of GdN. The relaxation time, $\tau$, can be estimated as follows. Since the crystallites are around $10 \mathrm{~nm}$, the mean free path can not be greater than this. At the room temperature we can calculate the velocity of free electrons by using $v=\left(3 k T / m^{*}\right)^{1 / 2}$. Assuming a mean free path of 1 to $10 \mathrm{~nm}$, the estimated relaxation time for an electron in conduction band comes out to be of the order $10^{-15}-10^{-14} \mathrm{~s}$. An estimate of the carrier density and the relaxation time can also be made from the optical data as described in the section 4.4. 
The calculated carrier density is a representative value of a weakly doped semiconductor.

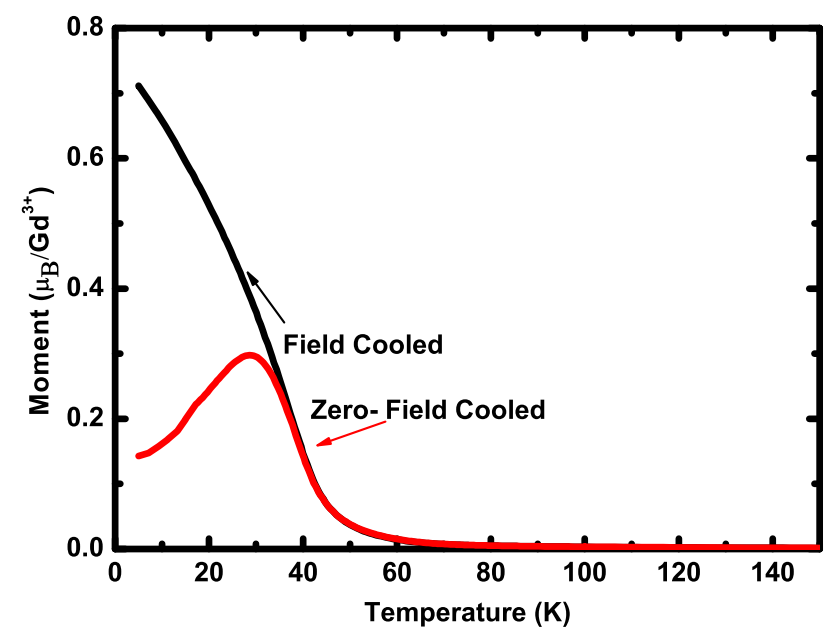

Figure 4.6 Magnetic moment of $G d N(G 6)$ as a function of temperature.

A plot of the magnetic moment vs temperature (Figure 4.6) measured in the absence of a magnetic field (Zero-Field Cooled) and then with an applied external field of 250 Oe (Field Cooled) shows that the magnetization rises in the vicinity of $50 \pm 1 \mathrm{~K}$ indicating the ferromagnetic Curie temperature is in this region.

A Curie - Weiss plot in Fig. 4.7 establishes a ferromagnetic transition temperature of $50 \pm 1 \mathrm{~K}$. This value of the Curie temperature, $\mathrm{T}_{C}$, reconciles well with the peak in resistivity which also occurs at the same value of the temperature. It therefore suggests that the resistivity change below the $50 \mathrm{~K}$ is due to a ferromagnetic transition. As stated in the introduction of this chapter, there are a wide number of reports quoting different values of the Curie temperature for this compound ranging from $30 \mathrm{~K}$ to $70 \mathrm{~K}$. The $\mathrm{T}_{C}$ value of $50 \pm 1 \mathrm{~K}$ in this work lies in the middle of this range.

More recent experimental data ${ }^{193}$ on the interplay between magnetism and the density of carriers in the ferromagnetic semiconductor $\mathrm{GdN}$ points towards the formation of the magnetic polarons centred on the nitrogen vacancies. Those data report a Curie temperature of $50 \pm 1 \mathrm{~K}$ for the carrier concentration of less than $10^{18} \mathrm{~cm}^{-3}$ in agreement with the present work. Each nitrogen vacancy binds two electrons in a singlet state with a third electron bound weakly below the conduction band. In a ferromagnetic phase, 


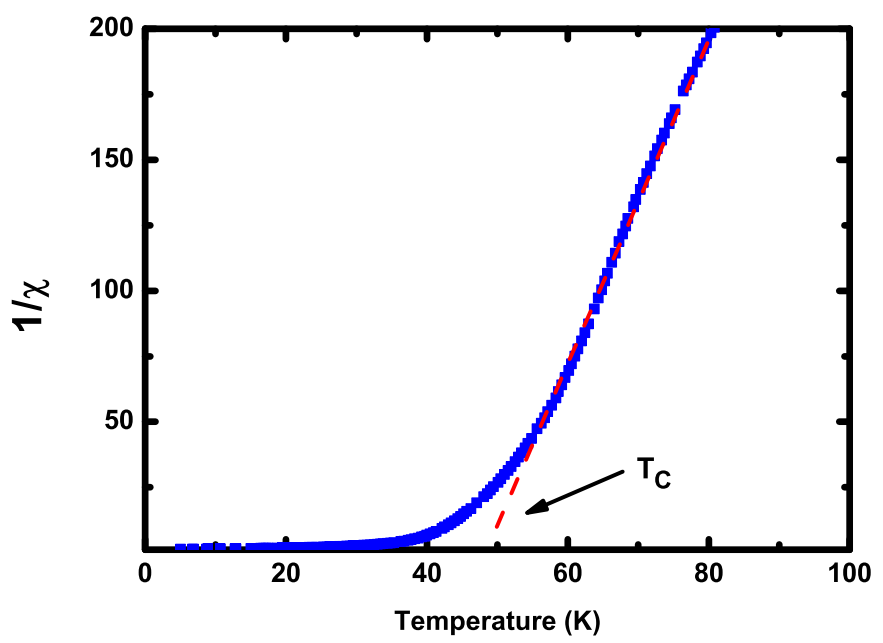

Figure 4.7 Curie - Weiss behaviour of a $G d N(G 6)$ film.

i.e. at temperatures below $\mathrm{T}_{C}$, carriers occupy the same level as the third electron and mediate exchange between the neighbouring Gd ions. Conduction electrons in an ionic crystal illustrate the qualities of a polaron. In GdN, these conduction electrons form the magnetic polarons. It is the density of nitrogen vacancies at which metal - insulator and $70-50 \mathrm{~K}$ ferromagnetic transitions occur. A theoretical report from Duan et al. ${ }^{194}$ predicts that the $\mathrm{T}_{C}$ would reduce with an increase in lattice constant. Since the reported GdN samples have an expanded lattice constant, our value of $\mathrm{T}_{C}$ is in line with this proposition. However, the epitaxially grown GdN films by Yoshitomi et al. ${ }^{98}$ which report almost the same value of lattice spacing as of this work (i.e. $0.504 \pm 0.003 \mathrm{~nm}$ ), report a $\mathrm{T}_{C}$ value of $30 \mathrm{~K}$, significantly smaller than our $50 \pm 1 \mathrm{~K}$ obtained on the polycrystalline films. There, the authors observed a sixfold symmetry as opposed to the threefold symmetry of rocksalt indicating the formation of an antiphase of GdN. Another explanation for the low paramagnetic to ferromagnetic transition temperature could be the oxygen contamination. ${ }^{121,122,131}$ No secondary phases in the films (Fig. 4.4) are detected but the possibility of slight oxidation of $\mathrm{GdN}$ at the film/cap interface exists as explained above. Interestingly our carrier concentration $\left(10^{18} \mathrm{~cm}^{-3}\right)$ and $\mathrm{T}_{C}$ reconcile well with the theoretical study of Sharma et al. ${ }^{192}$ Based on a modified RKKY theory, it predicted a transition temperature of $50 \mathrm{~K}$ for an assumed number of the carriers and the lattice constant that we report here. 
Hysteresis curves provide further evidence about the ferromagnetic state of GdN as shown in Fig. 4.8. A significant coercive field of 400 Oe was observed at $5 \mathrm{~K}$ along with the remnance of $1.3 \mu_{B} / \mathrm{Gd}^{3+}$. The estimated magnetic moment (inset Fig. 4.8) is around $5 \mu_{B} / \mathrm{Gd}^{3+}$, smaller than in the bulk $\mathrm{GdN}$ with a magnetic moment of $7 \mu_{B} / G d^{3+}$.

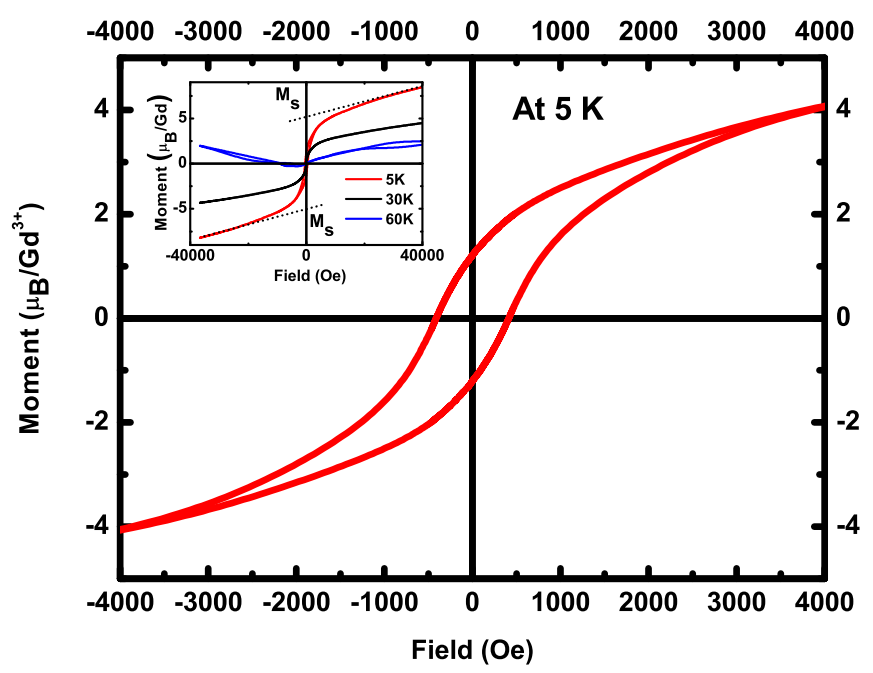

Figure 4.8 Hysteresis loop at $5 \mathrm{~K}$ for a $G d N(G 4)$ film. Inset shows the paramagnetic state of GdN even at $60 \mathrm{~K}$. 


\subsection{Optical Properties}

Optical transmittance $(\mathrm{T})$ and reflectance $(\mathrm{R})$ were measured in the region $0.5 \mathrm{eV}$ to $5.5 \mathrm{eV}$. The reflectance measurements were made on both sides of the sample, the substrate side and the cap side. Figures 4.9 and 4.10 show the $\mathrm{R}, \mathrm{T}$ and $\mathrm{R}+\mathrm{T}$ spectra of the sample $\mathrm{GdN}(\mathrm{G} 5)$ of thickness $\approx 740 \mathrm{~nm}$, grown at a high nitrogen partial pressure with a $\mathrm{N}_{2} / \mathrm{Gd}$ ratio of approximately 180 . This is a near stoichiometric sample with few nitrogen vacancies.

We begin our discussion with the infra red region. In the sub - gap region, below $1.0 \mathrm{eV}$, the average transmittance is nearly $85 \%$ and the reflectance is almost $10 \%$, variations being the effects of interference due to the multiply transmitted and reflected beams. It is immediately obvious that the absorptance $(A=1-R-T)$ is zero within uncertainty indicating a very low free carrier density and also indicating that there are no interband transitions in this energy range. Note that at $0.5 \mathrm{eV}$, the experimental $\mathrm{R}+\mathrm{T}$ curve exceeds unity slightly because of a systematic error in reflectance measurement. The average transmission and reflection in this range implies a real index of refraction, $n$, of the material being 2.0 by assuming that the imaginary part of the refractive index, $k=0$.

Above $1.0 \mathrm{eV}$, a rapid drop in the transmission is apparent signifying interband transitions. Since the $\mathrm{MgF}_{2}$ capping layer and the sapphire substrate are transparent in the energy range of our interest, signatures of the electronic transitions would only come from the GdN film. The fall in the transmittance continues until $5.5 \mathrm{eV}$ where it is less than $2 \%$.

To estimate the extinction coefficient, initially the spectral dependence of the refractive index was not allowed for and reflection/transmission data above the absorption edge was fitted. The frequency dependence of the refractive index was then allowed for. However the frequency dependence of the real part of the refractive index is very weak therefore we quote a refractive index of the GdN film as $2.0 \pm 0.1$. 


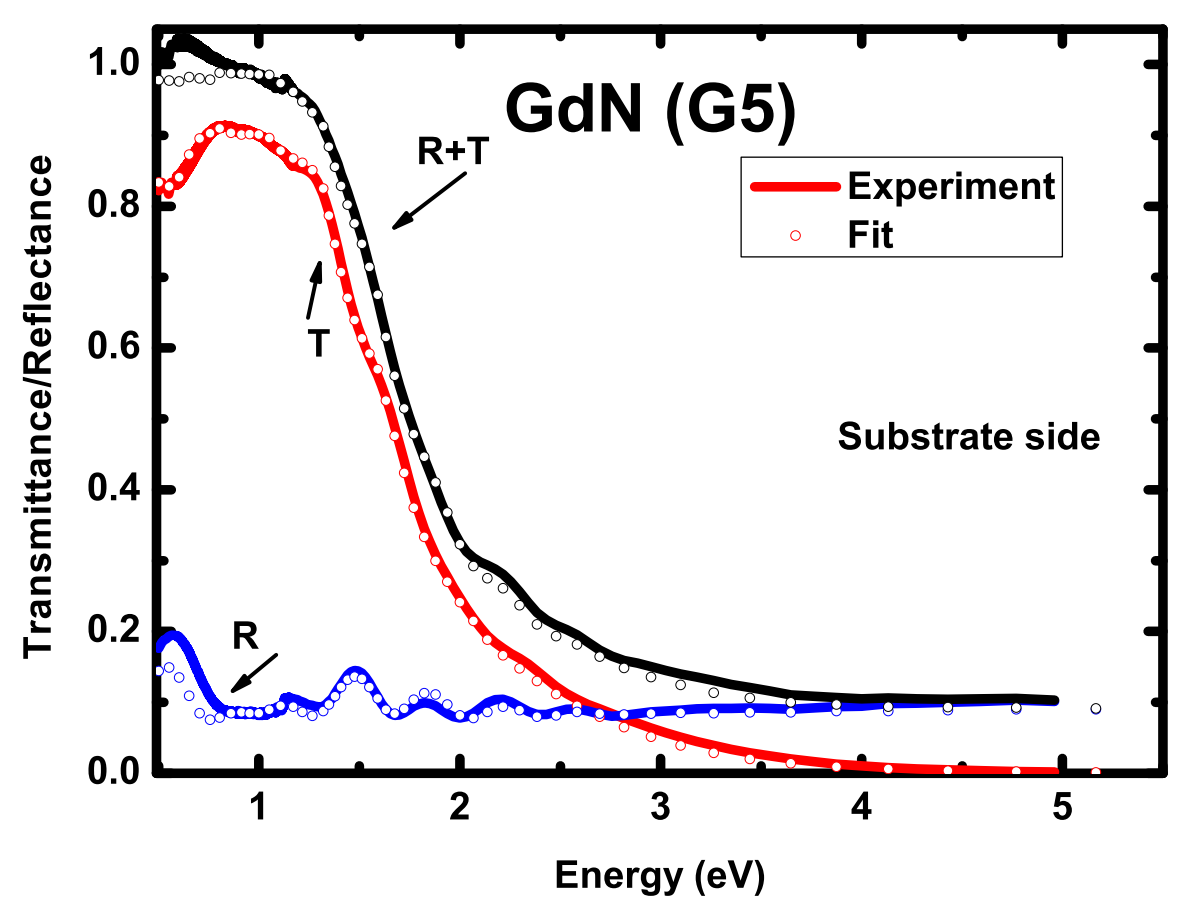

Figure 4.9 Reflectance $(R)$, transmittance $(T)$ and $R+T$ from the substrate side for the sample GdN (G5) thin film. Open circles represent simulated spectra whereas solid lines are experimental.

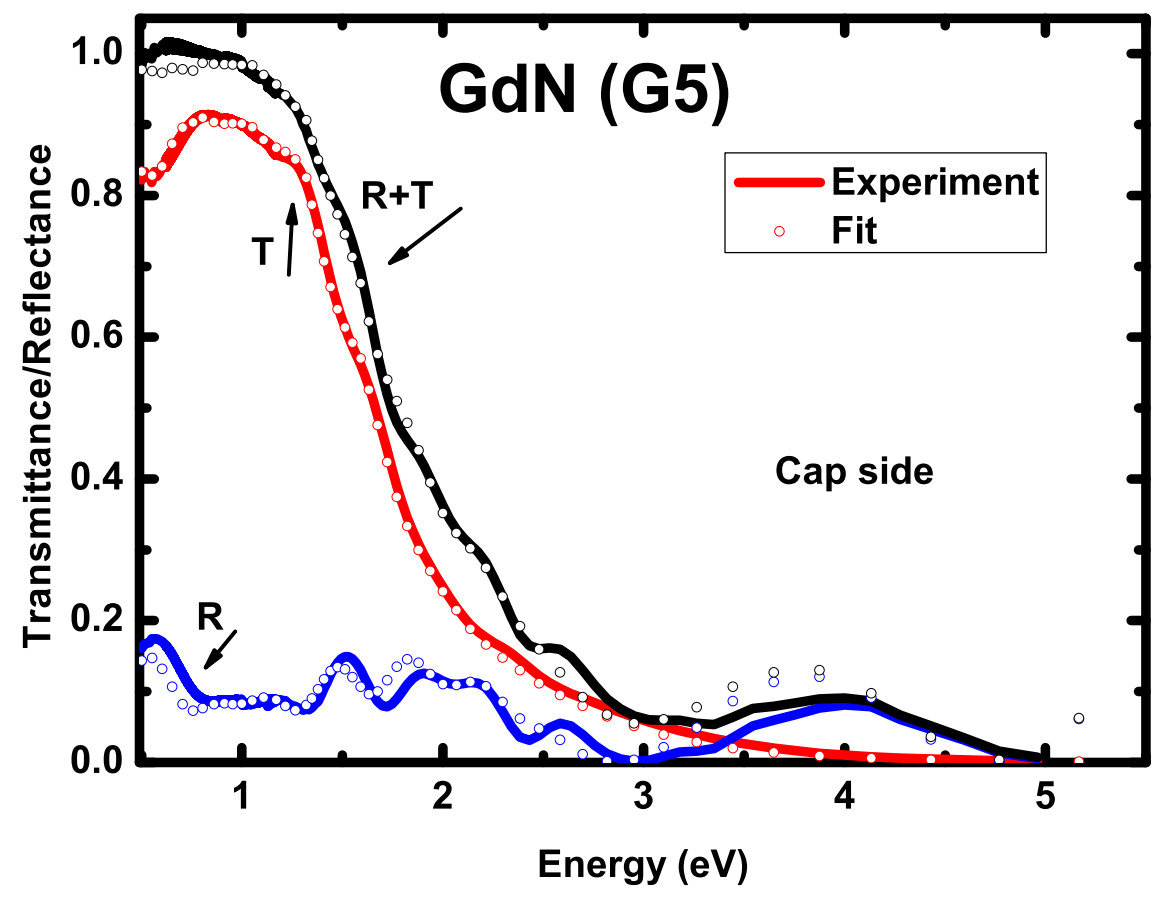

Figure 4.10 $R, T$, and $R+T$ spectra from the capping side of the sample $G d N(G 5)$. 
The open circles in figures 4.9 and 4.10 show the fitted transmission and reflection compared to the experimental curves. The agreement between the experimental and the simulated spectra is excellent. There is, however, a small inconsistency in the positions of the crests and troughs between the measured and fitted spectra. The computer program, TFCalc $^{175}$, assumes perfectly parallel air/cap/film/substrate/air interfaces, however, a certain degree of the interface roughness is expected from the actual samples resulting in the displaced interference positions between the simulated and experimental spectra.

The density of free carriers was determined in the region of transparency by using ${ }^{169,171}$ :

$$
\alpha=\frac{4 \pi}{\lambda}\left(\frac{\sigma_{D C}}{2 n \varepsilon_{0} \omega^{3} \tau^{2}}\right)
$$

where $\alpha$ is the absorption coefficient, $\sigma_{D C}$ is the DC conductivity, $\tau$ is the relaxation time, $\varepsilon_{0}$ is the permittivity of the free space, $\omega$ is the angular frequency and $n$ is the refractive index. A transmittance spectrum was then generated using

$$
T=(1-R) e^{-\alpha d}
$$

which showed an average transmission of $85 \%$ establishing the number of carriers of the order $10^{18} \mathrm{~cm}^{-3}$, in agreement with the carrier density calculated by the resistivity measurement.

Note that the shape of the reflectance spectra are different above $2.5 \mathrm{eV}$ from the substrate and cap side. The interference pattern from the cap side of the film is broad due to the thin capping layer. In this region, most of the light is absorbed (more than $90 \%$ ) so the reflectance is only contributed by the capping layer material, $\mathrm{MgF}_{2}$, of refractive index 1.4. The uneven form of the interference is due to the reflectance from the capping layer and the film, both. The substrate, on the other hand, is a single crystal with a refractive index of 1.8 therefore the reflectance remains more or less at $10 \%$. 
A plot of the extinction coefficient, as obtained by fitting the experimental reflection/transmission spectra, as a function of energy (Fig. 4.11) shows an onset of absorption at $1.3 \mathrm{eV}$. The inset shows the absorption coefficient, calculate using $\alpha=4 \pi k / \lambda$, rising sharply above $10^{4} \mathrm{~cm}^{-1}$, a characteristic of direct interband electronic transitions in a semiconductor. A possible effect of weak free carrier absorption can be seen below the absorption edge, although this could just be the result of a poor fitting in this region and is below the confidence level of $5 \%$.

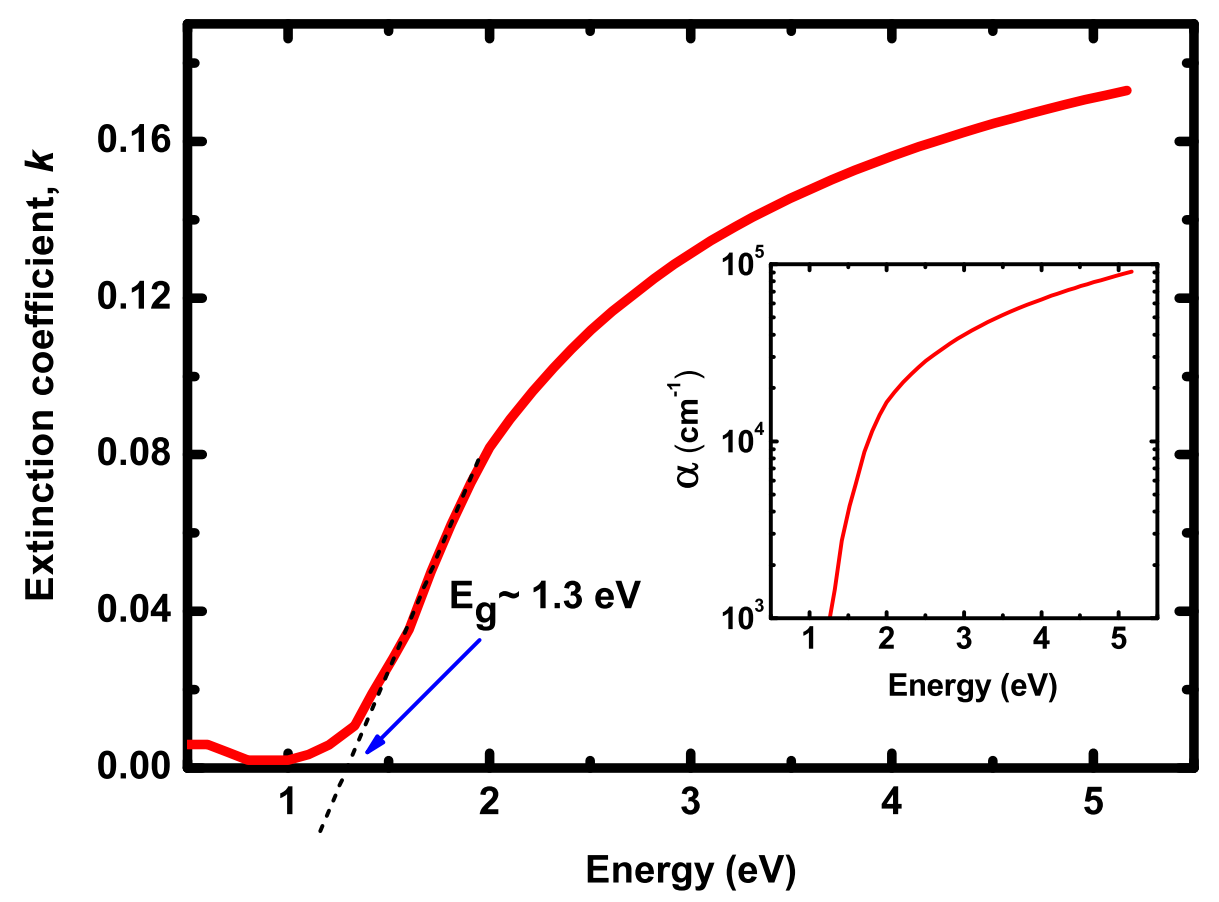

Figure 4.11 Imaginary part of the complex refractive index for a $G d N(G 5)$ sample. Inset shows the absorption coefficient. 
For the sake of comparison, $\mathrm{R}, \mathrm{T}$ and $\mathrm{R}+\mathrm{T}$ spectra from the cap side of a relatively thinner film, around $300 \mathrm{~nm}$, are shown in Fig. 4.12. This sample was also prepared at a higher $\mathrm{N}_{2} / \mathrm{Gd}$ flux ratio therefore it is expected to be near stoichiometric. Here, while the absorption edge is quite broad, the average transmission and reflection are the same as in Fig. 4.11 and 4.10, providing further the confirmation that the refractive index of GdN thin films is around $2.0 \pm 0.1$. Again note that $\mathrm{R}+\mathrm{T}$ in these spectra exceeds unity because of an uncertain reflectance measurement. It is clear from the optical data that there is no indication of a metallic character of the films with no Drude response, therefore it is in good agreement with the semiconducting model.

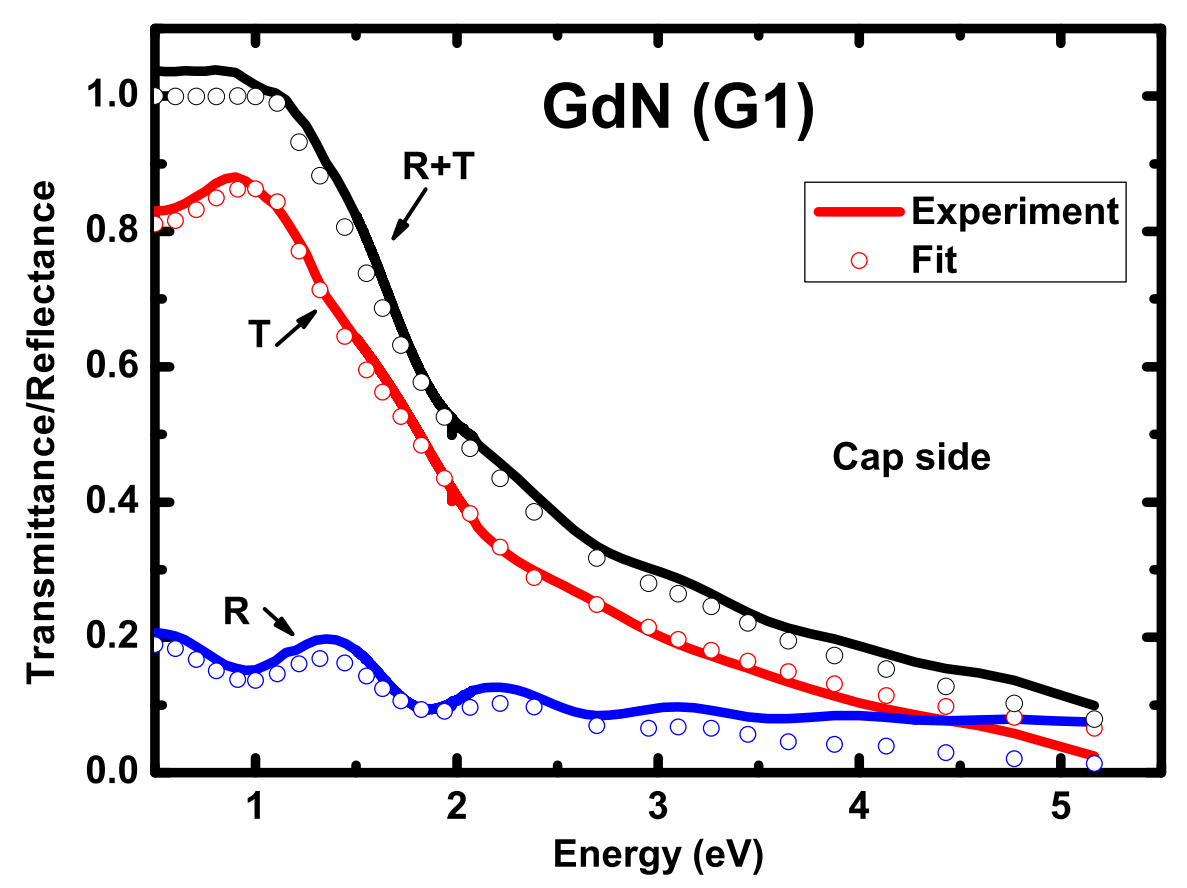

Figure 4.12 $R, T$ and $R+T$ spectra from the substrate side of the film $G d N(G 1)$. 
Figure 4.13 shows the real and imaginary parts of the dielectric function calculated from $n$ and $k$ obtained from the fitting of R-T spectra. The real part of the complex dielectric function, $\varepsilon^{\prime}=n^{2}-k^{2}$, shows only weak frequency dependence whereas the imaginary part, $\varepsilon^{\prime \prime}=2 n k$, is monotonically increasing with the energy. The imaginary part of the dielectric function is almost directly proportional to the joint density of states (Eq. 2.22) if the matrix element for the transition is independent of the position in k-space on the surface that defines energetically allowed transitions. There is no discernible structures shown by $\varepsilon^{\prime \prime}$ in the high energy region which would indicate an onset of the absorption at any other energy, thus validating the optical energy gap in $\mathrm{GdN}$ at $1.3 \pm 0.05 \mathrm{eV}$. These features are consistent with those expected for a semiconductor.

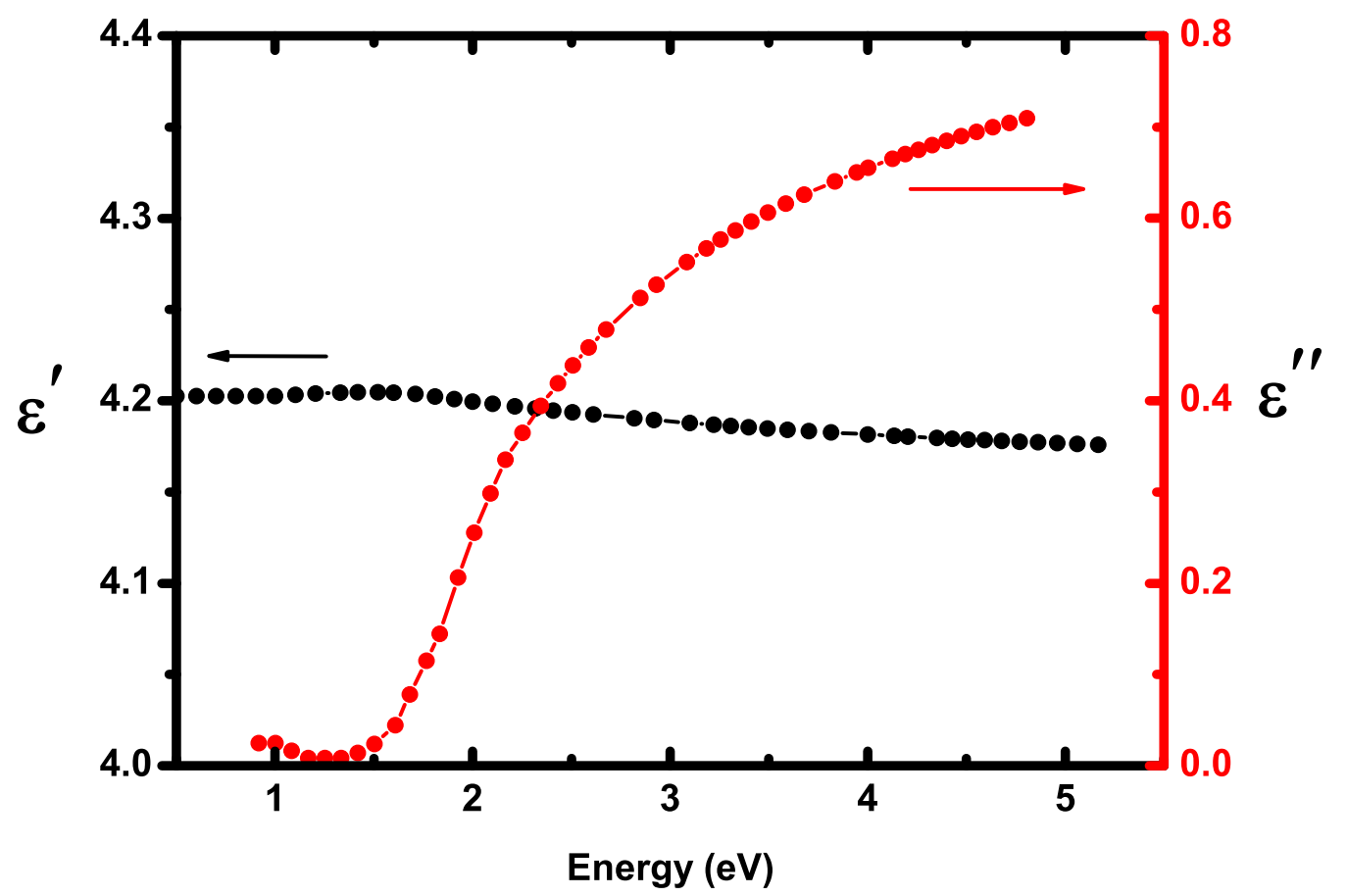

Figure 4.13 Real and imaginary parts of the dielectric function.

The onset of absorption in this work is smaller than what was observed by Schneemeyer et al. ${ }^{122}$ The absorption edge, as shown by the fall in percentage transmittance in their work, is quite broad making the accurate determination of the onset of absorption difficult.

Further, on the experimental front, there is a disagreement of the present results with those of Yoshitomi ${ }^{97,98}$ et al. which show a dependence of absorption edge on the film thickness. Their estimate of the energy gap is $1.29 \mathrm{eV}$ and $1.18 \mathrm{eV}$ for epitaxial films of 
thickness $30 \mathrm{~nm}$ and $95 \mathrm{~nm}$ respectively. Their Curie temperature of $30 \mathrm{~K}$ is also lower than that measured for the GdN thin films in the present work.

On the other side, present optical measurements are in substantial agreement with the observations of Trodahl et al. ${ }^{1}$ The optical energy gap and the absorption coefficient are in compliance but also the room temperature resistivity of $0.14 \Omega \mathrm{cm}$ agrees well with their $0.15 \Omega \mathrm{cm}$, thus the carrier density is also the same. A major difference, however, is in the value of the Curie temperature which in the present case is $20 \mathrm{~K}$ less than their 70 K. It is known ${ }^{137,192}$ that excessive free carriers would increase the Curie temperature whereas it is low for near stoichiometric films.

In the case of the dielectric function, our measurements are not in firm agreement with the low frequency dielectric constant of 6.71 as theoretically predicted by Xue et al. ${ }^{167}$ The present work will also show that for the case of $\mathrm{DyN}$, the dielectric function as inferred from the experimental data in the near IR region is in ample agreement with the far IR values. ${ }^{100}$ Since DyN and GdN have very similar band structures ${ }^{2}$, it is expected that the dielectric function would not show any remarkable difference for GdN. The refractive index value of the present work is also significantly different from 3.3 as calculated by Mitra et al. ${ }^{120}$ Also their calculated absorption coefficient is substantially larger than that measured in this experimental work. 


\subsection{Conclusion}

Near stoichiometric films of GdN, varying in thickness, were prepared under an ultra high vacuum environment and protected with $\mathrm{MgF}_{2}$ or GaN layers. A resistivity of $0.14 \Omega \mathrm{cm}$, establishes a carrier density of order $10^{18} \mathrm{~cm}^{-3}$ with an assumed value of relaxation time, $\tau=10^{-14}-10^{-15} \mathrm{~s}$, typical for a semiconductor. Magnetization measurements show a small value of the magnetic moment and a high coercive field. The Curie - Weiss law is obeyed in its paramagnetic state but the prepared GdN films exhibit a magnetic transition temperature around $50 \mathrm{~K}$, less than the generally accepted value of $70 \mathrm{~K}$ but greater than the recently published $30 \mathrm{~K}$. The semiconducting nature of the GdN thin films, as manifested by the resistivity measurements, is further confirmed by optical transmittance and reflectance measurements. A clear fundamental absorption edge at $1.3 \pm 0.05 \mathrm{eV}$ is observed in accordance with theory and previous experiments. ${ }^{1}$ The absorption coefficient rises sharply to values above $10^{4} \mathrm{~cm}^{-1}$, typical for a semiconductor. Fitting of the experimental R-T spectra gives a refractive index of $2.0 \pm 0.1$ which is in agreement with the refractive index of $\mathrm{DyN}^{100}$ with a similar band structure. 


\section{Chapter 5}

\section{Low Temperature Response of GdN Transmission Spectra}

In ferromagnetic semiconductors, the absorption edge shifts from the short wavelength part of the spectrum to the long wavelength as the temperature drops below $\mathrm{T}_{C}$, an effect known as a red - shift of the absorption edge. ${ }^{195}$ Associated with magnetic ordering, the red - shift of the absorption edge is observed in $3 d$ transition metal oxides. ${ }^{157,196-198}$ For the case of GdN, the theoretically predicted ${ }^{2}$ ferromagnetic red - shift of the absorption edge was confirmed experimentally by Trodahl et al. ${ }^{1}$ The band structure calculations were then tuned to the experimental data which, with the choice of a screened Coulomb potential $U_{d}=8.0 \mathrm{eV}$ in an $\mathrm{LSDA}+U$ approximation, gave an average energy gap of $1.30 \mathrm{eV}$ and a majority - spin gap of $0.91 \mathrm{eV}$. The shift of the minority and the majority spin states in the conduction and the valence bands was predicted to be in the opposite directions. As a result, the bottom of the majority/minority states conduction band minimum is closer to the majority/minority states valence band maximum. Figure 5.1 shows an expanded view of the band structure as calculated by Trodahl et al. ${ }^{1}$ illustrating the energy gap between the majority spin states of about $0.9 \mathrm{eV}$ and $1.7 \mathrm{eV}$ for the minority spins.

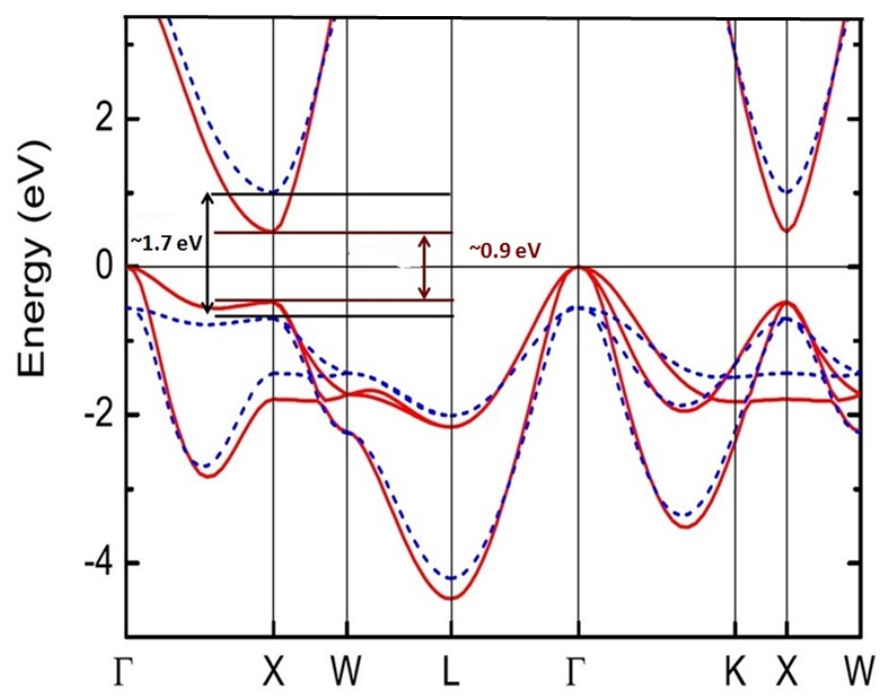

Figure 5.1 An expanded view of the band structure of GdN calculated ${ }^{1}$ by the LSDA+U approach. The solid red lines represent majority spins and dashed blues lines represent the minority states. 
The determination of the dielectric response function by Mitra and Lambrecht ${ }^{120}$ was then based on these experimental observations made by Trodahl. ${ }^{1}$ The calculated real and the imaginary parts of the dielectric function were significantly different for the spin up and the spin down states in the near energy gap region indicative of spin polarization in both the conduction and the valence bands.

In the last couple of years, further experimental accounts of magneto-optical effects of GdN have come forward. ${ }^{97-99,166}$ A gradual increase in the band gap has been observed by Yoshitomi et al. as the temperature reduces to $\mathrm{T}_{C}$ from $300 \mathrm{~K}$ whereas a dramatic reduction was observed below $\mathrm{T}_{C}$, attributed to the short range and long range spin correlation respectively. A relation of the carrier scattering with the spin splitting energy difference between the minority and majority bands has been studied by Vidyasagar et al. ${ }^{166}$ pointing towards the spin splitting in the conduction and valence band as the origin of ferromagnetism in GdN. The spin splitting in GdN was further studied by Yoshitomi et al. ${ }^{99}$ by employing temperature dependent circularly polarized spectroscopy. The band gap energy trends appeared to be different for right circularly polarized (RCP) and left circularly polarized (LCP) light. The band gap energy decreases for the RCP with the decrease in temperature (red - shift) but the opposite happens for the LCP for the temperature approaching $\mathrm{T}_{C}=30 \mathrm{~K}$. A spin splitting energy difference, $\Delta \mathrm{E}$, is calculated from the difference in optical band gaps computed from RCP and LCP.

Against this background, it is clearly interesting to study the low temperature optical response of GdN to develop further understanding of its ground state nature. The presentation of the spin polarized optical structure of GdN thin films is the objective of this chapter. Optical transitions between the electronic states of $\mathrm{GdN}$ in its ferromagnetic phase will be highlighted in a manner similar to the paramagnetic GdN phase. The transmission spectra during the phase transition of GdN will be analysed first. The discussion will be carried forward to the determination and interpretation of the optical constants and the dielectric function in a magnetically ordered GdN. The implications of the transitions from the paramagnetic to the strong ferromagnetic state on the joint density of states will be discussed at the end. 


\subsection{Temperature Dependent Transmission Spectra}

Transmission spectra at variable temperature were obtained by using a Bomem DA 8 FTIR spectrometer in the range $0.5-2.1 \mathrm{eV}$ on a $775 \mathrm{~nm}$ thick $\mathrm{GdN}(\mathrm{G} 6)$ film grown at a high $\mathrm{N}_{2}$ partial pressure $\left(10^{-4}\right.$ mbar) as described in detail in chapter 3 . The film was grown on a sapphire substrate and protected by a $\mathrm{MgF}_{2}$ layer.

Frequency dependent transmission spectra at different temperatures are shown in Fig. 5.2. The temperature range of $140 \mathrm{~K}$ to $55 \mathrm{~K}$ is represented by a single spectrum at $140 \mathrm{~K}$ as they appeared to be indistinguishable within the experimental uncertainty. As discussed in the previous chapter, an almost flat transmission except for the interference fringes shows no evidence of absorption in the subgap region.

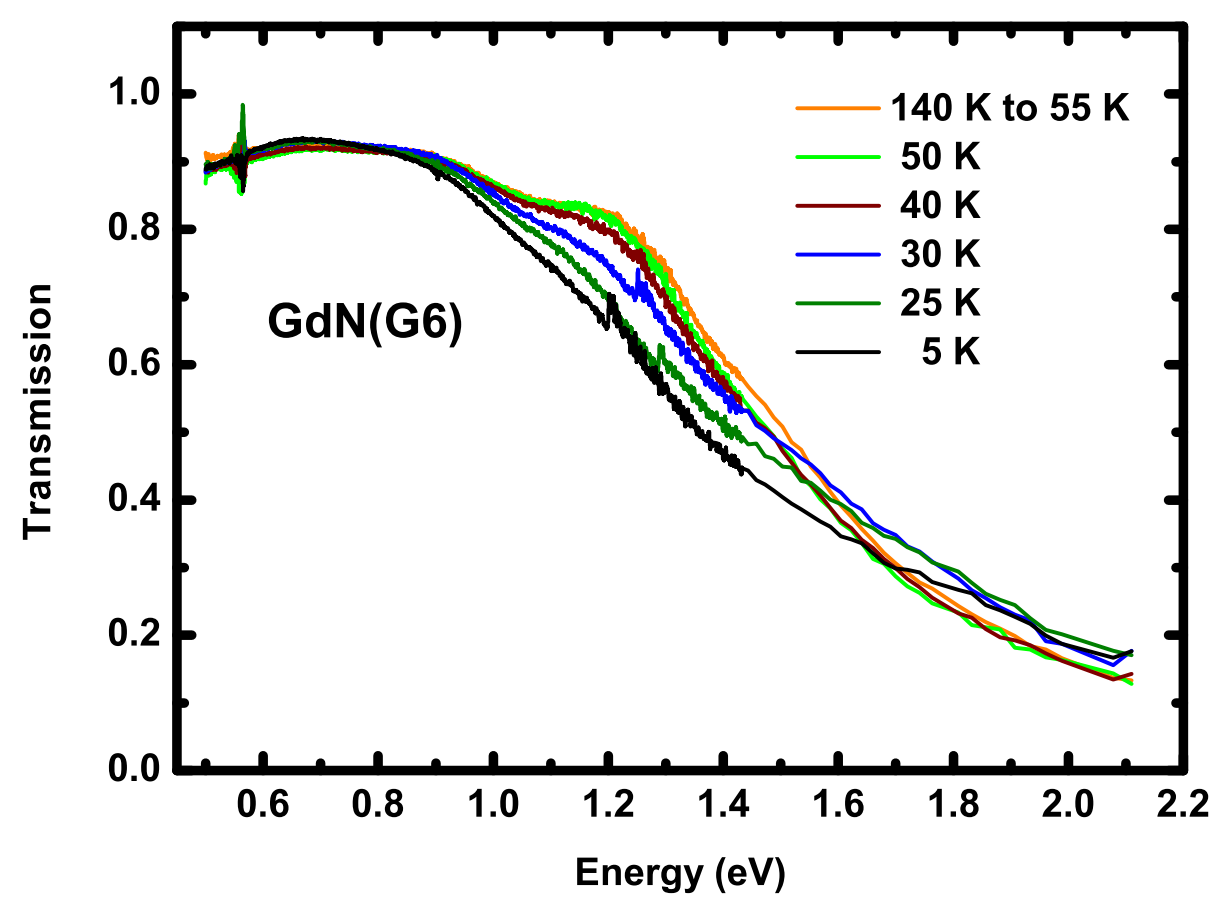

Figure 5.2 Transmission spectra at different temperatures from a $G d N(G 6)$ sample.

An interesting feature, immediately noticeable, is that the material is transparent from $0.5 \mathrm{eV}$ to $0.8 \mathrm{eV}$ for all temperature ranges, with no perceptible electronic transitions in this range. The spectra obtained below $50 \mathrm{~K}$ show a gradual decrease in the transmission above $0.8 \mathrm{eV}$ and the fundamental absorption edge tends to shift towards the lower part of the energy spectrum. Note that $50 \pm 1 \mathrm{~K}$ is the Curie temperature as exhibited by magnetic 
measurements on the GdN films reported in this work. The direct gap shrinkage at the $\mathrm{X}$ point continues as the temperature is reduced further in the ferromagnetic state of $\mathrm{GdN}$ and the $5 \mathrm{~K}$ transmission spectrum is clearly distinct from the rest displaying an absorption edge below $0.90 \mathrm{eV}$.

Another notable aspect, shown by the transmission spectrum at $5 \mathrm{~K}$, is a cross over at just above $1.6 \mathrm{eV}$. The spectrum shows a higher transmission of the light (low absorption) above $1.6 \mathrm{eV}$ compared to the rest which continues to fall below $20 \%$. This characteristic is shared by all transmission spectra collected below the Curie temperature. We will associate this feature, later, with the spin-splitting of the joint density of states. Data on the low temperature transmission from the other GdN samples are shown in Fig. 5.3 where they display similar trends.
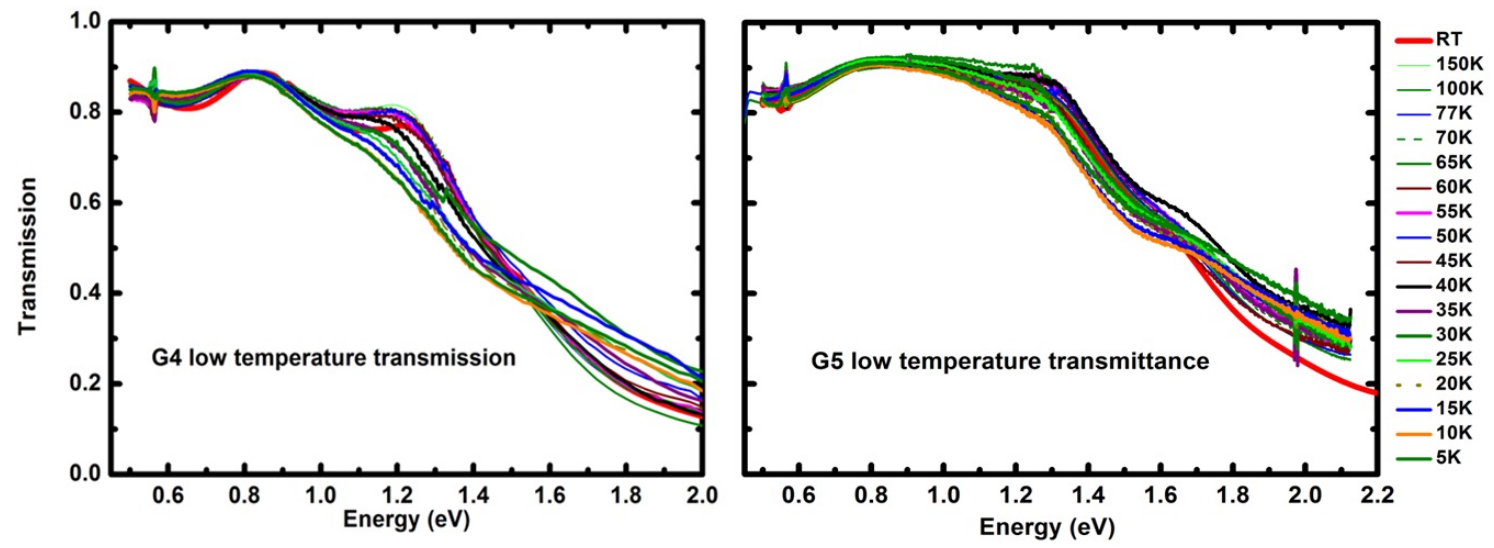

Figure 5.3 Temperature dependent spectra for GdN-G4 and G5 samples. 


\subsection{Temperature Dependent Extinction Coefficient}

Figure 5.4 shows the extinction coefficient of $\mathrm{GdN}(\mathrm{G} 6)$ in its ferromagnetic phase at the $5 \mathrm{~K}$ and also in the paramagnetic state at $140 \mathrm{~K}$ in the photon energy range $0.5 \mathrm{eV}-2.1 \mathrm{eV}$. For the sake of clarity only two spectra are being shown here. Note that fitting of the low temperature transmission spectra was done with real index of refraction $2.0 \pm 0.1$ for $\mathrm{GdN}$, unchanged from its room temperature value. As is also apparent from the transmission spectra in Fig. 5.2, the fundamental absorption edge at $5 \mathrm{~K}$ in Fig. 5.4, in the ferromagnetic phase of $\mathrm{GdN}$, is the red-shifted to $0.87 \pm 0.05 \mathrm{eV}$ compared to its paramagnetic phase at $140 \mathrm{~K}$ which is at $1.25 \pm 0.05 \mathrm{eV}$. For $300 \mathrm{~K}$ (Fig. 4.11 of chapter 4 ) this value is approximately $1.30 \pm 0.05 \mathrm{eV}$. The uncertainty is shown only for the subgap region by the error bars in Fig. 5.4 for the $k$ spectrum at $140 \mathrm{~K}$, with non zero values for $k$ depicting a finite absorption due to free carriers.

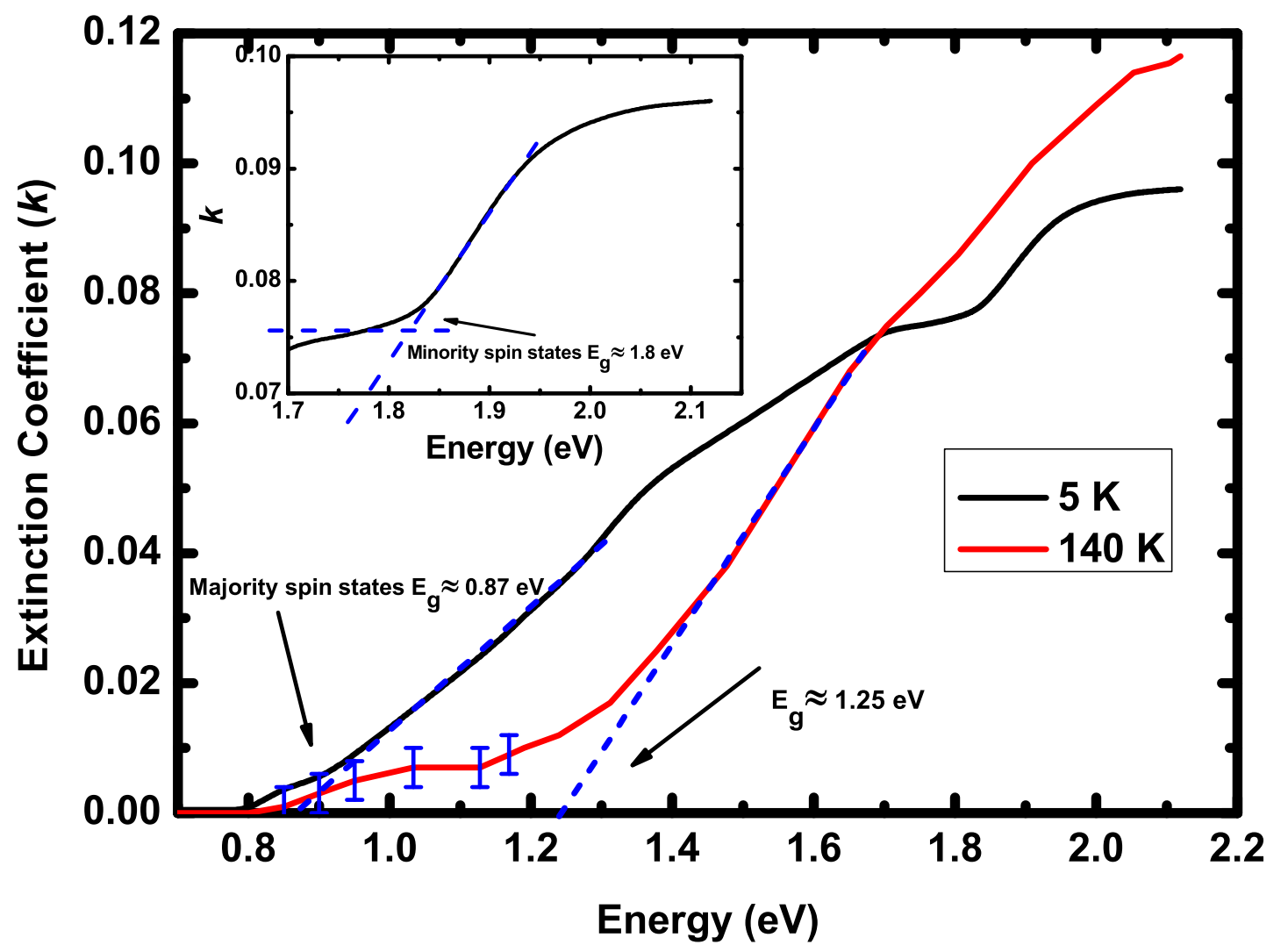

Figure 5.4 Extinction coefficient of $G d N(G 6)$ in its ferromagnetic phase at $5 \mathrm{~K}$ and in its paramagnetic state at $140 \mathrm{~K}$.

The energy gap values were obtained by extrapolating the $k$ spectrum (dashed lines) as 
shown in Fig. 5.4. The other prominent feature in the $k$ spectrum at $5 \mathrm{~K}$ appears at just below $1.8 \mathrm{eV}$ (inset Fig. 5.4). There is clearly the presence of another electronic transition at this energy value in contrast to $140 \mathrm{~K}$ where the absorption increases with the increase in the energy without any significant change. Note that below $1.8 \mathrm{eV}$ and above $1.6 \mathrm{eV}$, the absorption is nearly levelled as if saturating. Its position is in line with the transmission measurements shown in Fig. 5.2. Also note that the absorption for the spectrum at $5 \mathrm{~K}$ is significantly higher compared to the $140 \mathrm{~K}$ spectrum below $1.6 \mathrm{eV}$ after which a prominent reduction in the absorption can be viewed. Above $1.90 \mathrm{eV}$, the extinction coefficient, again, tends to be flat. Such behaviour is not clear in the paramagnetic phase of GdN.

Figure 5.5 shows the imaginary part of the dielectric function, $\varepsilon^{\prime \prime}$, calculated from the extinction coefficient. Correlation between the electronic spins in the ferromagnetic phase becomes stronger as the temperature goes below $50 \mathrm{~K}$, the Curie temperature. As discussed in chapter 2 , the imaginary part of the complex dielectric function $\varepsilon^{\prime \prime}$ is directly related to the joint density of states (Eq. 2.21) so these direct electronic transitions, at low and at high energies, in the ferromagnetic phase of GdN can be assigned to the spin up and spin down (majority and minority) electrons.

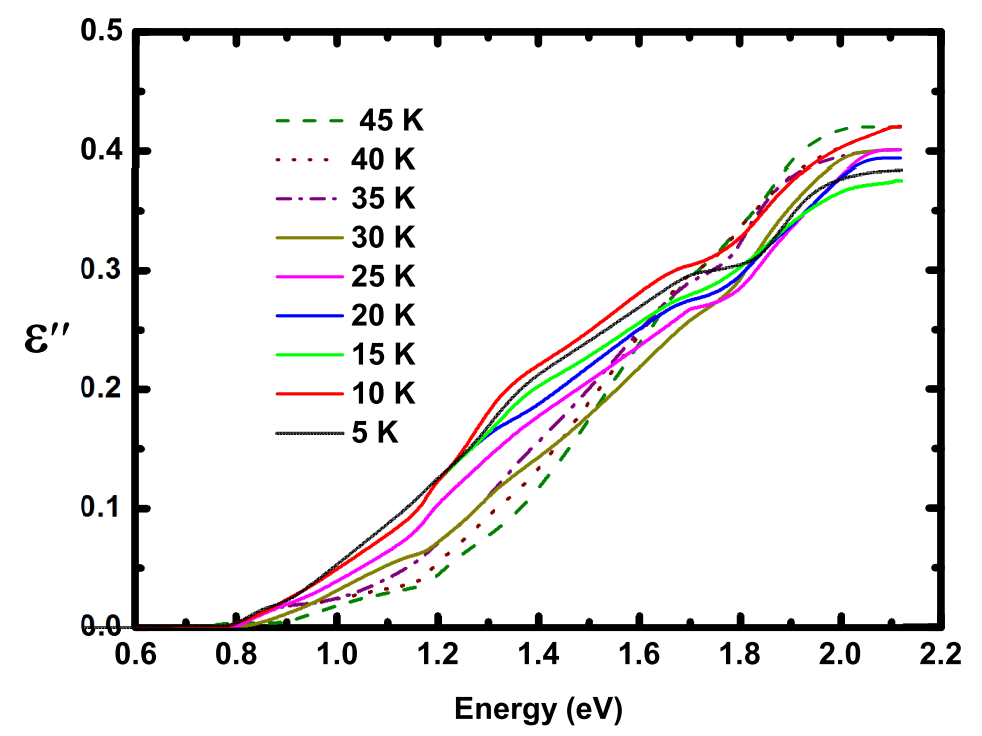

Figure 5.5 Evolution of imaginary part of the dielectric function with temperature for a GdN sample. 
Figure 5.6 shows the direct energy gap values for the minority spins (solid red circles) and the majority spins (solid black squares), obtained by extrapolating the imaginary part of refractive index as a function of temperature. Solid blue squares represent the magnetization. At about $50 \pm 1 \mathrm{~K}$, when the magnetization rises steeply, a clear blue shift and a red shift is visible for the minority and the majority spin states, respectively. At a temperature of $5 \mathrm{~K}$, the energy difference between the minority and majority spins is nearly $0.90 \mathrm{eV}$, in agreement with the $0.90 \pm 0.03 \mathrm{eV}$ as was observed by Trodahl et al. ${ }^{1}$ (Fig. 5.1), which begins to narrow down as the temperature increases. In addition, an average measured energy gap of $1.70 \pm 0.05 \mathrm{eV}$ between minority spin states is also in complete agreement with the band structure of GdN in Fig. 5.1. Above $50 \mathrm{~K}$, the Curie temperature, the energy gap is significantly narrowed showing that the exchange splitting between the minority and the majority spins is weak. The average energy of both spins is shown in the middle (solid green triangles) which fluctuates about the mean value of $1.3 \pm 0.1 \mathrm{eV}$, representing value of the direct energy gap at the $\mathrm{X}$ point for $\mathrm{GdN}$ in its paramagnetic state as measured by reflectance/transmittance spectra in chapter 4 .

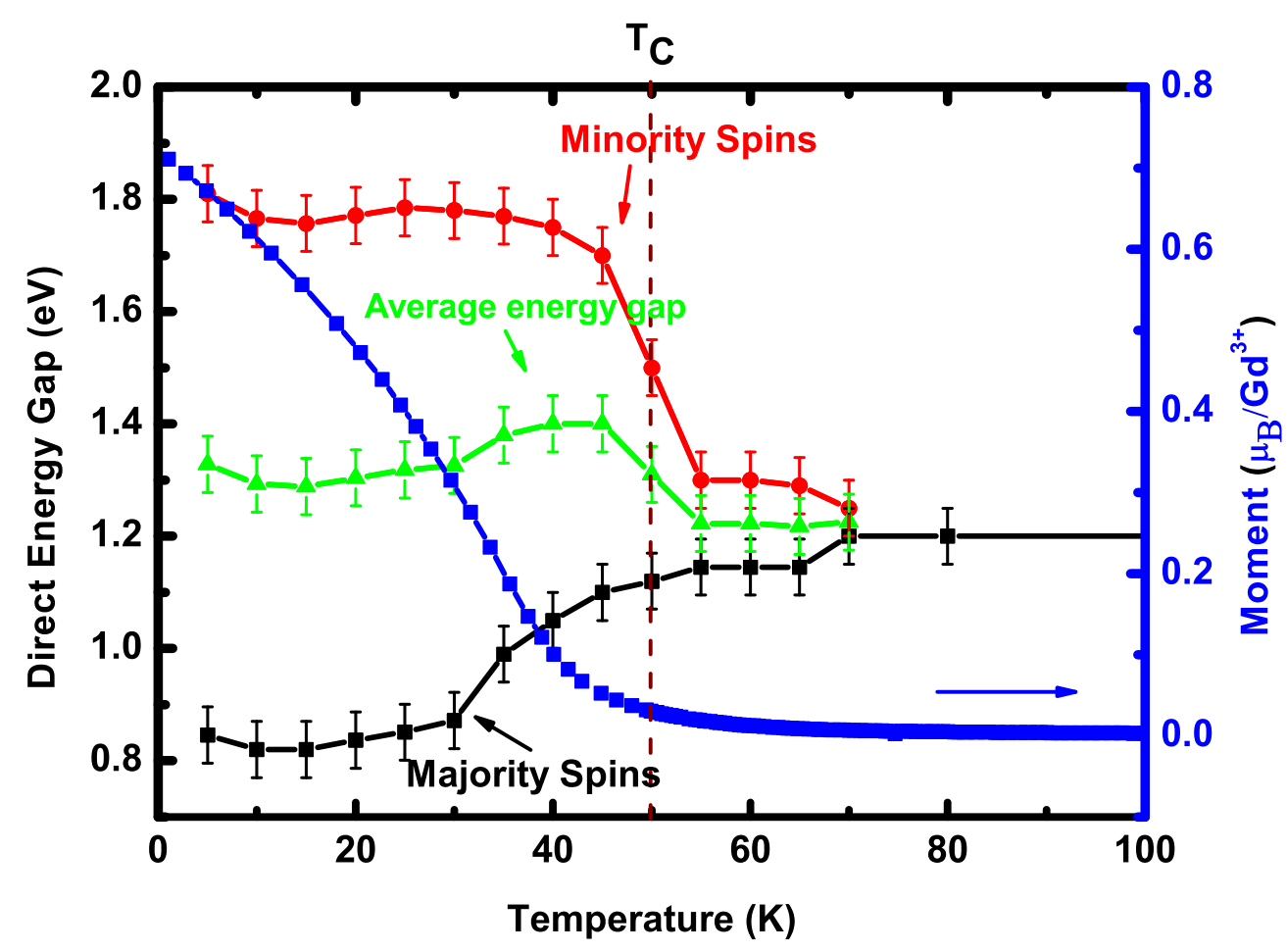

Figure 5.6 Energy difference between minority and majority spin states for ferromagnetic $G d N$. 


\subsection{Conclusion}

The transmission spectra for GdN films in their ferromagnetic phase was collected. The amount of light transmitted in the energy range $0.5 \mathrm{eV}$ to $0.8 \mathrm{eV}$ remains unchanged at all temperatures, including the para - and ferromagnetic phases, indicating that no electronic transitions are present in this energy region. Low temperature transmission spectra were fitted to obtain the extinction coefficienst with real refractive index $2.0 \pm 0.1$. The onset of absorption shifts to the lower energy as the temperature is decreased from $300 \mathrm{~K}$. Below $\mathrm{T}_{C}=50 \pm 1 \mathrm{~K}$, two clear absorption edges are observed attributed to the spin splitting of electron states in the valence and the conduction bands. The majority spin states show a red - shift in the energy, while a blue - shift for the minority states is observed. The experimental observations presented in this work are in good agreement with the previously published results of Trodahl et al. ${ }^{1}$ although the work is extended to measure both the majority and minority spin states. The presented measurements reaffirm that GdN is ferromagnetic at low temperature with an energy gap of $0.90 \pm 0.05 \mathrm{eV}$ for majority spin states and $1.70 \pm 0.05 \mathrm{eV}$ for minority spins. 


\section{Chapter 6}

\section{Dysprosium Nitride}

With an electronic configuration of [Xe] $4 f^{10} 6 s^{2}$, dysprosium (Dy) is also prized for its magnetic properties. Both experimental and theoretical studies on its nitride compound report widely deviating claims for its optical electronic structure. Calculations of the electronic structure ${ }^{111}$ of DyN using the SIC-LSD method showed that there are no bands at the Fermi energy and therefore it is a small gap insulator a gap of $0.24 \mathrm{eV}$ for the spin-up states, while for the spin-down states it is $0.78 \mathrm{eV}$ in ferromagnetic state. Like the band structure of GdN, no $f$ levels occur at the Fermi level. The LSDA+U approach predicted $^{2}$ that DyN has seven spin-majority $4 f$ states in three deep narrow bands. The remaining two $f$ electrons go in the minority spin bands at about 4.6 and $5.4 \mathrm{eV}$ below the top of the valence band (Fig. 6.1). A small indirect gap between the top of the valence band at $\Gamma$ and the conduction band minimum at $\mathrm{X}$ and a minimum direct gap of $1.17 \mathrm{eV}$ at $\mathrm{X}$ is also predicted by this model. Note that these calculations were performed assuming $U_{d}=6.4 \mathrm{eV}$ for the $d$ orbitals of dysprosium. Such calculations ${ }^{2}$ for the case of GdN resulted in an underestimated energy gap.

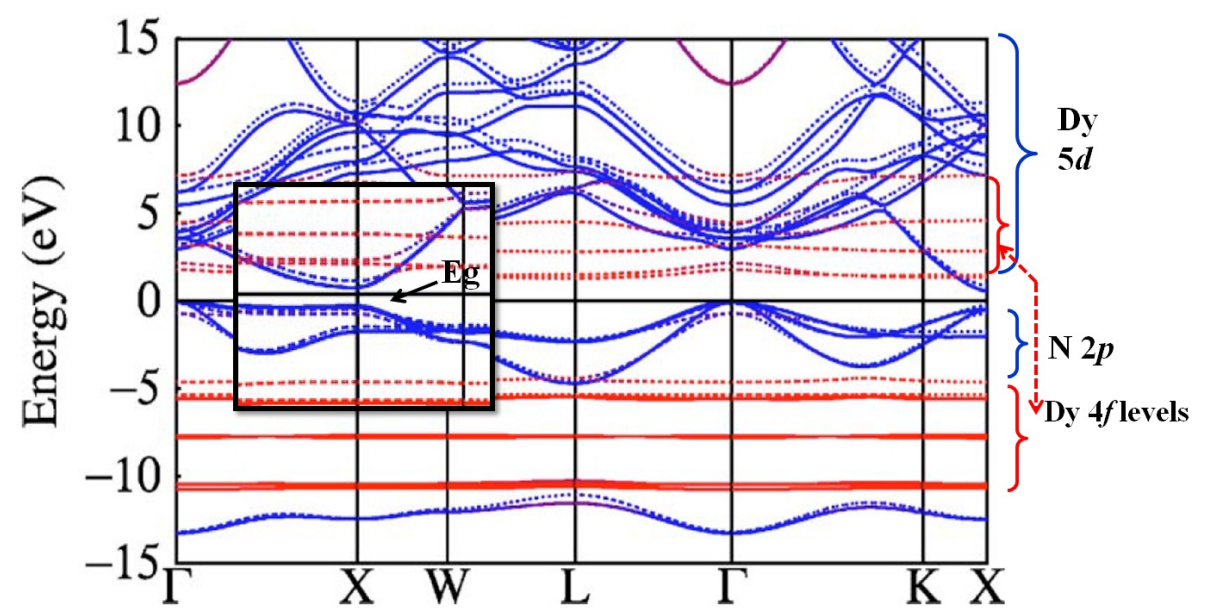

Figure 6.1 Band structure of DyN calculated by Larson et al. ${ }^{2}$ The dashed and solid blue lines are for majority and minority spins. Red solid and dashed lines represent filled and empty 4 flevels. Inset shows an enlarged view of the direct energy gap at the $X$ point. 
There are decades old experimental reports ${ }^{77,107}$ on the band gap of DyN, measured from the optical absorption of thin films with the gap is stated to be $0.91 \mathrm{eV}$ and $0.95 \mathrm{eV}$. Sclar et al. on the other hand reported a wide band gap from $2.60 \mathrm{eV}$ to $2.90 \mathrm{eV}$ depending on the substrate. ${ }^{78}$ Recently, Preston et al. measured an energy gap of $1.5 \mathrm{eV}$ from X-ray absorption and emission spectra on a thin film of DyN. ${ }^{112,147}$

In the wake of these reportedly different energy gap values, DyN emerges as an interesting member of the rare earth nitride family. Therefore there is a pressing need for a systematic experimental study of DyN in order to establish its true nature and electronic structure. This work presents the first ever reflection/transmission measurements carried out on reliable DyN thin films in the photon energy range of $0.5-5.0 \mathrm{eV}$. The optical energy gap is determined for the first time and evidence for the optical transitions above the gap is sought. The optical constants and the dielectric functions are also calculated using the characteristic matrix method. As a supporting evidence, the electronic and the magnetic measurements are also presented.

\subsection{Preparation of DyN Thin Films}

Thin films of DyN were prepared by thermal evaporation of Dy in an ultra high vacuum environment in the presence of $99.9 \%$ pure nitrogen gas and were protected by $\mathrm{MgF}_{2}$ layers. Boats of tantalum and tungsten were used to evaporate dysprosium and $\mathrm{MgF}_{2}$, respectively. Both metals, Ta and $\mathrm{W}$, have very low vapour pressure at the high temperatures $\left(10^{-4}\right.$ torr at nearly $\left.3000 \mathrm{~K}\right)$ and high melting points. The temperature, for a Dy vapour pressure of $10^{-4}$ torr is almost $1200 \mathrm{~K}$ and for the same vapour pressure of $\mathrm{MgF}_{2}$, it is $1800 \mathrm{~K}$ (Table 3.1 in section 3.1.1). Four films were prepared in total under varying conditions. The growth conditions are summarized in the Table 6.1. Note that the films D2 and D3 were prepared at a higher base pressure, of the order $2.0 \times 10^{-7}$ mbar and $1.0 \times 10^{-7}$ mbar respectively compared to D1 and D5 which were grown at a lower base pressure of $1.0 \times 10^{-8}$ mbar. 
Table 6.1 Growth Parameters of DyN Thin Films grown at Room Temperature

\begin{tabular}{|c|c|c|c|c|}
\hline $\begin{array}{c}\text { Sample } \\
\text { ID }\end{array}$ & $\begin{array}{c}\text { Growth } \\
\text { Pressure } \\
(\mathrm{mbar})\end{array}$ & $\begin{array}{c}\text { Deposition } \\
\text { Rate } \\
(\AA / \mathrm{s})\end{array}$ & $\begin{array}{c}\text { Film } \\
\text { Thickness } \\
(\mathrm{nm})\end{array}$ & $\begin{array}{c}\mathrm{N}_{2} / \mathrm{Dy} \\
\text { Flux ratio }\end{array}$ \\
\hline \hline D1 & $1.2 \times 10^{-4}$ & 0.5 & 420 & 158.75 \\
\hline D2 & $6.8 \times 10^{-5}$ & 2.0 & 304 & 22.10 \\
\hline D3 & $1.7 \times 10^{-4}$ & 1.5 & 320 & 75 \\
\hline D5 & $1.9 \times 10^{-4}$ & 0.5 & 287 & 258.75 \\
\hline
\end{tabular}

As discussed in the section 4.1, a higher nitrogen partial pressure ensures near stoichiometric films whereas nitrogen vacancies are more likely to take place at low nitrogen partial pressure. Another important parameter is the thickness of the grown films. The film thickness necessarily effects the number of interference fringes as well as attenuation of the transmitted light. $\mathrm{N}_{2}$ /Dy flux ratios and rates are calculated by using Eqs. 3.4 and 4.1. Since the thermal evaporation was controlled by a variable power supply, the growth pressure and the deposition rate could not be maintained at exactly the same value. Therefore the average growth pressure and the average deposition rate is reported in the Table 6.1. All of the films were grown at the room temperature on double side polished, c-plane (0001), sapphire wafers $\left(\mathrm{Al}_{2} \mathrm{O}_{3}\right)$ with pre-deposited silver $(\mathrm{Ag})$ contact pads. In - situ resistance measurements were made by using these contact pads which were connected to a digital multimeter. The film thickness was verified by the Rutherford back scattering spectrometry. 
Figure 6.2 shows the reciprocal of the resistance, measured in - situ, as a function of film thickness. An almost linear form of the plot confirms a nearly uniform growth of the DyN thin film. The slope of the plot, $d(1 / R) / d z$, where $R$ is the resistance in ohms and $z$ is the thickness in $\mathrm{cm}$, is directly related to the resistivity of the film. The width of the contact pads and the separation between them was nearly $1 \mathrm{~mm}$. The resistivity calculated from Fig. 6.2 is $1.4 \pm 0.03 \mathrm{~m} \Omega \mathrm{cm}$.

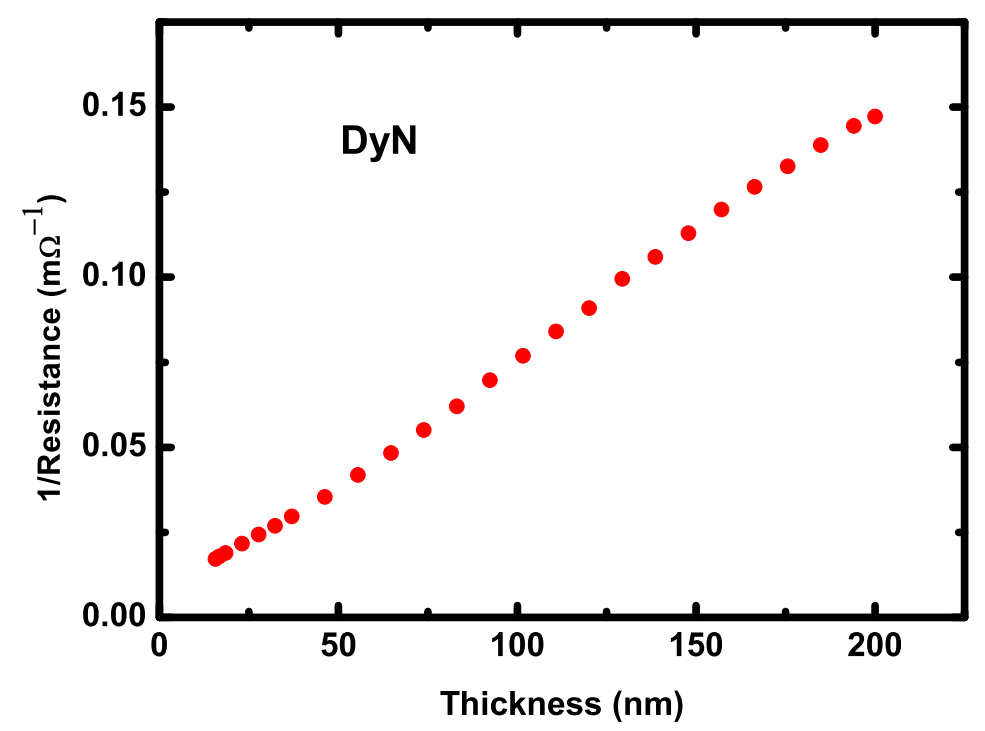

Figure 6.2 Reciprocal of film resistance vs thickness for DyN(D5) establishes uniform growth of the film prepared at room temperature. 


\subsection{Structural Characterization}

The XRD scan of a representative DyN thin film, deposited on a sapphire substrate and passivated by a $\mathrm{MgF}_{2}$ capping layer is shown in Fig. 6.3. The strongest peak from the sapphire substrate stands out. Next, the most noticeable peak labelled as (111) and a rather weak (222) peak are attributed to the cubic structure of DyN. It is clear that the film is strongly textured in the $<111>$ direction. The lattice constant of the films is $4.970 \pm 0.003$ And the average crystallite size is about $10 \mathrm{~nm}$ as obtained using the Scherrer formula (Eq. 3.8). This value of the lattice constant is slightly larger than the previously reported value $68,80,112,147$ of $4.895 \AA$. Also the crystallite size reported for the present sample is larger than $8 \mathrm{~nm}$, the one reported by Preston et al. ${ }^{112,147}$ despite the use of the same growth technique.

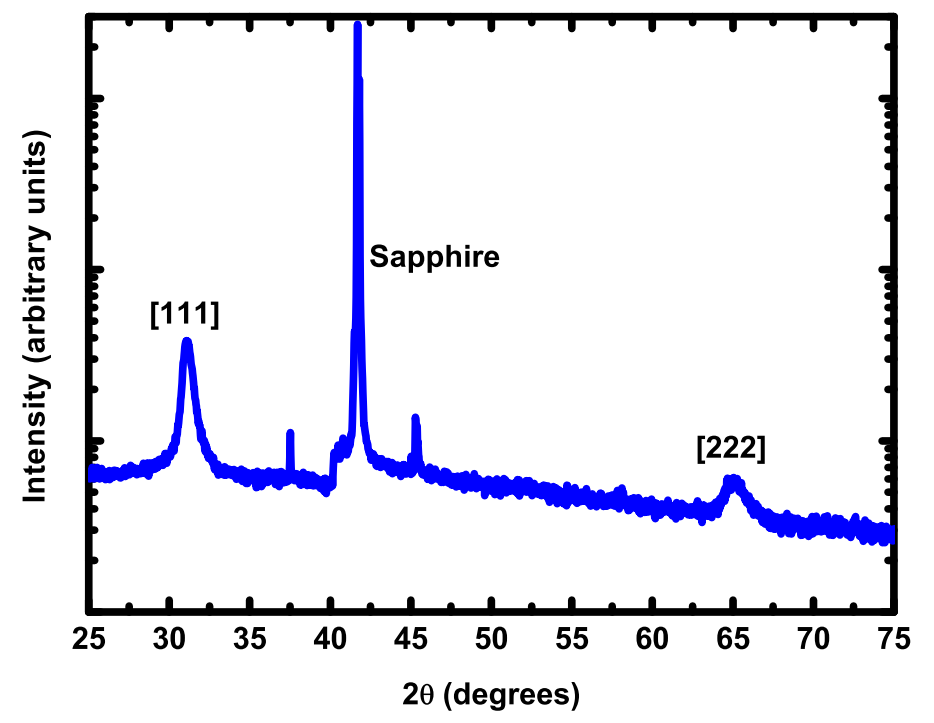

Figure 6.3 XRD pattern for a representative DyN(D5) sample. 


\subsection{Temperature Dependent Resistivity and}

\section{Magnetization}

Figure 6.4 shows the temperature dependent resistivity of a film grown under the similar conditions as of DyN(D5) but with a GaN capping layer. The semiconducting nature of the film is confirmed by strongly rising resistivity with a decreasing temperature. A relatively flat peak near the ferromagnetic Curie temperature is followed at lower temperature by a continuation of the rise, affirming a semiconducting ground state below $\mathrm{T}_{C}$. An ambient resistivity value of $100 \mathrm{~m} \Omega \mathrm{cm}$ and an estimated relaxation time of $10^{-14}$ to $10^{-15} \mathrm{~s}$ leads to a calculated carrier concentration of less than $10^{20} \mathrm{~cm}^{-3}$, characteristic of a moderately doped semiconductor.

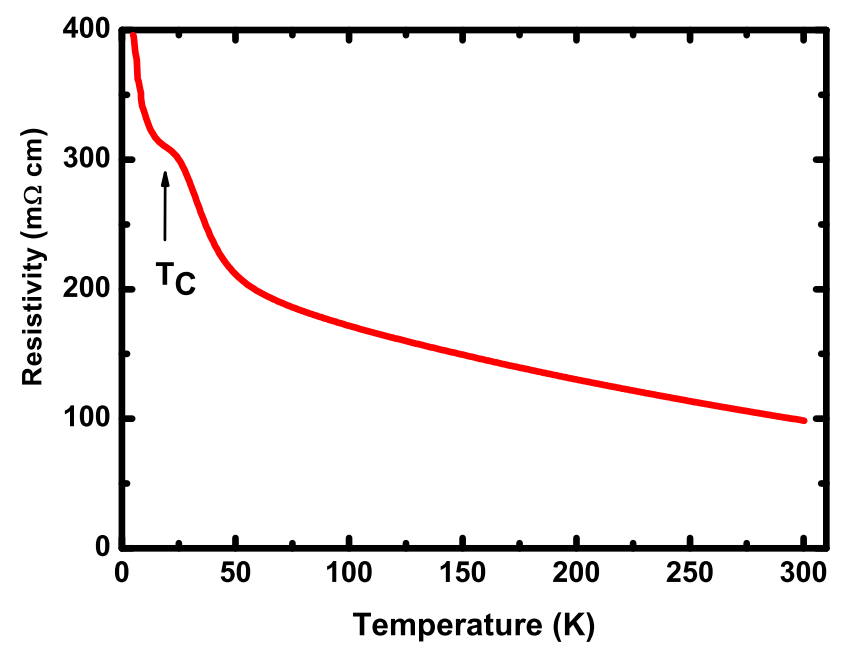

Figure 6.4 Temperature dependent DC resistivity of a DyN thin film with a GaN cap, grown under the similar conditions as of DyN(5). 
Turning to the magnetization, in Fig. 6.5, a divergence between the field cooled (FC) and zero - field cooled (ZFC) data below $25 \mathrm{~K}$ implies a ferromagnetic transition.

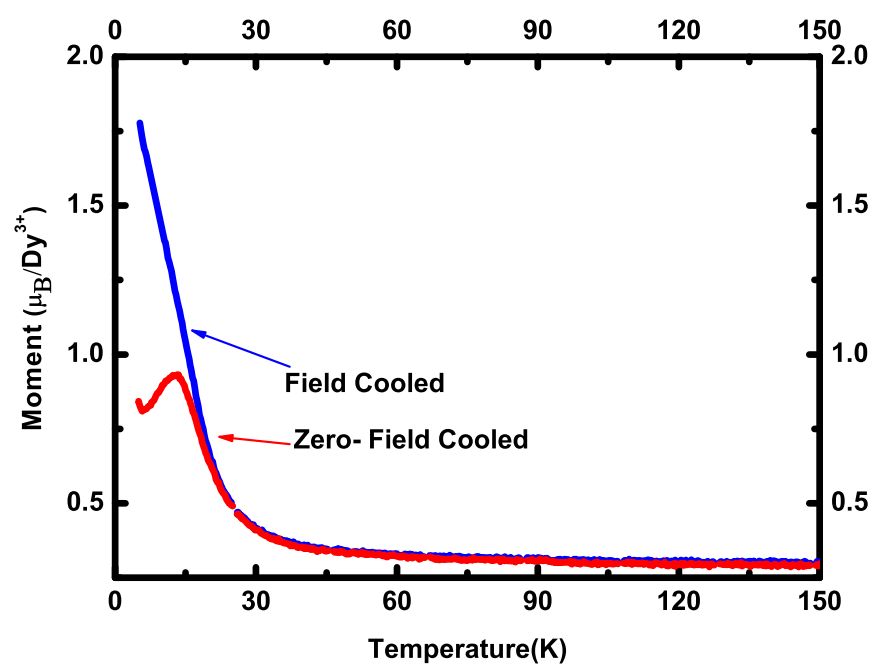

Figure 6.5 Magnetization as a function of temperature for a $D y N(D 4)$ sample.

Figure 6.6 shows the hysteresis curves of a DyN thin film illustrating a high moments of around 9.7 $\pm 0.3 \mu_{B} / \mathrm{Dy}^{3+}$ which is close to $10 . \pm 0.3 \mu_{B} / \mathrm{Dy}^{3+}$ the one found in literature. ${ }^{81}$ Clearly the DyN thin film is in its ferromagnetic phase, as depicted by the hysteresis curve at $5 \mathrm{~K}$. At a higher temperature however the hysteresis is not very clear indicating a Curie temperature in the vicinity of $20 \pm 1 \mathrm{~K}$.

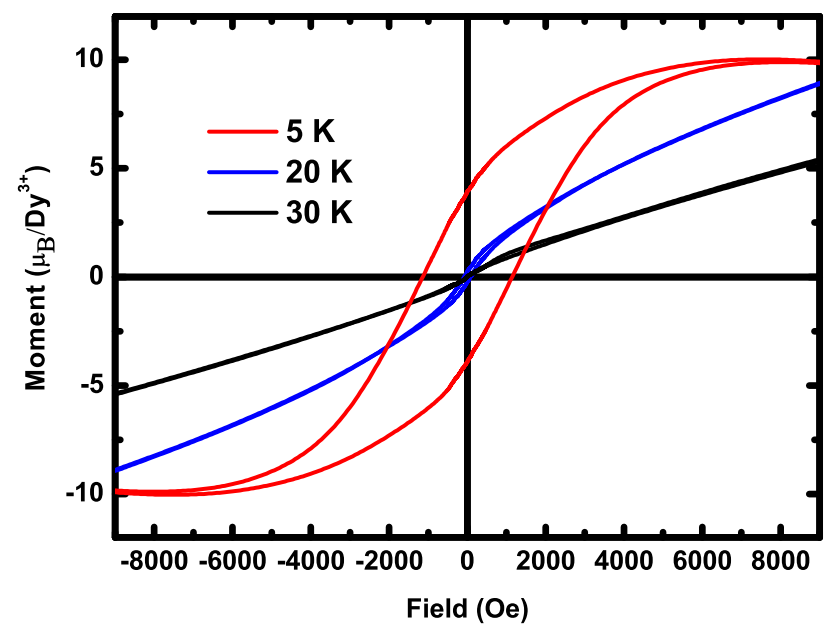

Figure 6.6 Hysteresis curves at low temperatures for the sample DyN(D5) which is in its ferromagnetic state at and below $20 \mathrm{~K}$. 
A Curie - Weiss plot (Fig. 6.7) depicts a $\mathrm{T}_{C}$ of around $20 \pm 1 \mathrm{~K}$, which is lower than the value $25 \mathrm{~K}$ reported by Preston et al. ${ }^{112}$ Nevertheless, it lies in the range of $17 \mathrm{~K}$ to $25 \mathrm{~K}$, the reported values of $\mathrm{T}_{C}$ for $\mathrm{DyN}{ }^{80,81,150,151}$

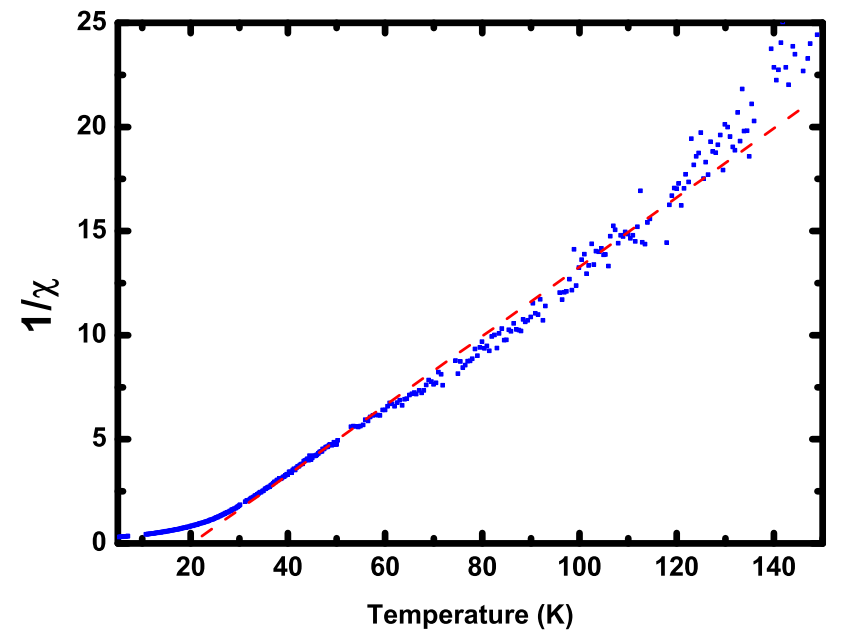

Figure 6.7 Curie - Weiss plot for a DyN(D3) sample. 


\subsection{Optical Properties}

We choose to report the reflection/transmission spectra and their sum, R+T, of two films, with one near stoichiometric while other is affected by nitrogen vacancies. Figures 6.8 \& 6.9 show the R-T spectra from a $287 \mathrm{~nm}$ thick film of DyN, sample D5, which was prepared at the highest $\mathrm{N}_{2} /$ Dy flux ratio as depicted in Table 6.1. Consider the low energy region, the region below $1.0 \mathrm{eV}$, first. In this region, the absorptance $(\mathrm{A}=1-\mathrm{R}$ T) is zero with $2 \%$ uncertainty, establishing a very low free carrier density as expected of a semiconductor and signalling that this energy range is below the interband edge. The effect of interference fringes in both the reflection and transmission spectra in this region can readily be seen. Above $1.2 \mathrm{eV}$ the transmitted light falls gradually indicating the presence of interband transitions which continues to fall till $5.0 \mathrm{eV}$ where it is less than $1 \%$.

Further the interpretation of the R-T spectra was accomplished assuming the refractive indices for $\mathrm{MgF}_{2}$ capping layer and sapphire $\left(\mathrm{Al}_{2} \mathrm{O}_{3}\right)$ substrate as 1.4 and 1.8, respectively. To first approximation, the absorption below the edge was initially set to zero, as is any case indicated by the $\mathrm{R}+\mathrm{T}=1$. The refractive index of 2.0 was then determined by fitting the transmission spectra below the band edge; even the average value of transmission ensures that this is the refractive index in this energy range. Next, with this value of the refractive index approximated as constant above the edge, values of $k$ were extracted by fitting the absorption spectra. The spectral dependence of the refractive index was then allowed, but the variations were below the level of confidence so we quote a refractive index of $2.0 \pm 0.1$. The open circles in the figures show a comparison between the calculated and the measured R-T spectra. We regard the fit as reasonable; the computer program, TFCalc, calculated optical spectra for perfect interfaces and uniform films, but in reality one expects the films to show some degree of interface roughness. 


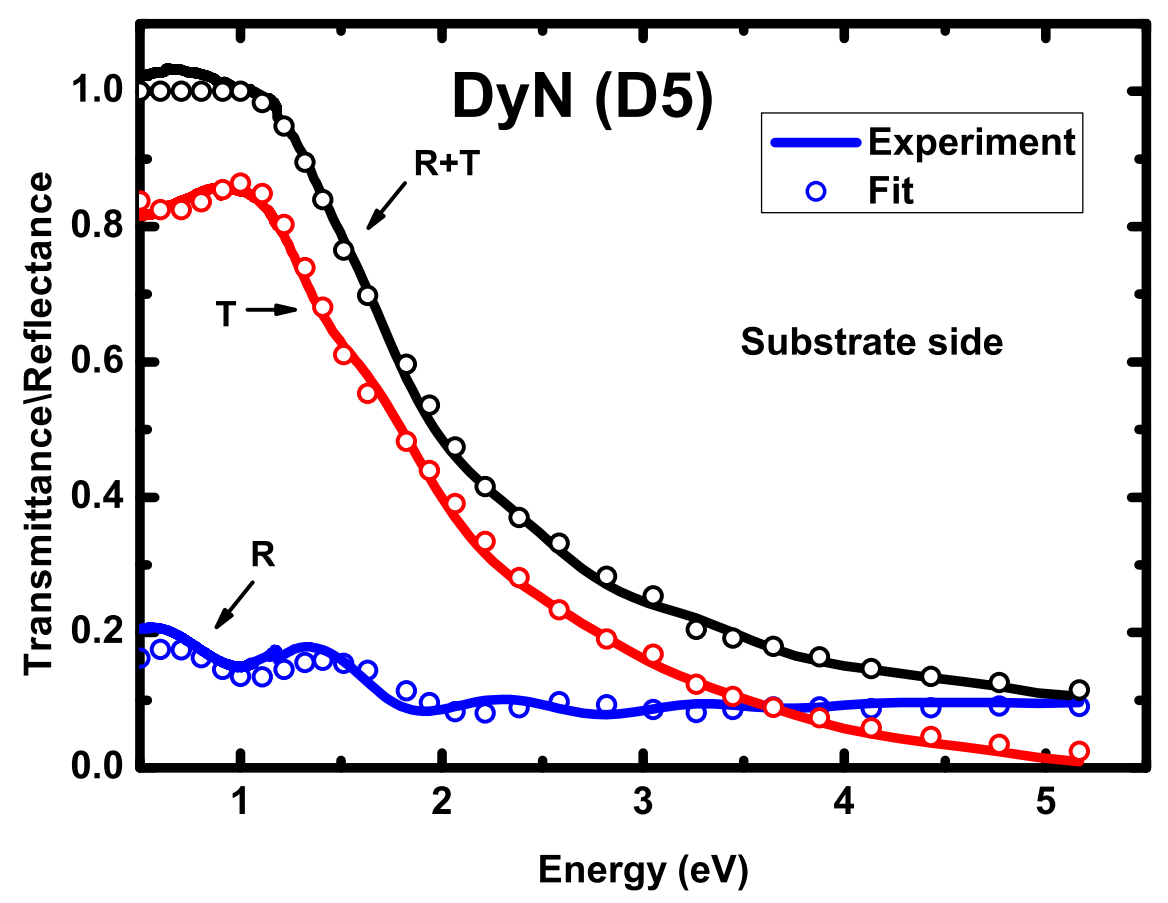

Figure 6.8 $R, T$ and $R+T$ from the substrate side of $D y N(D 5)$ thin film. Solid lines are experimentally obtained spectra whereas open circles represent fitted spectra.

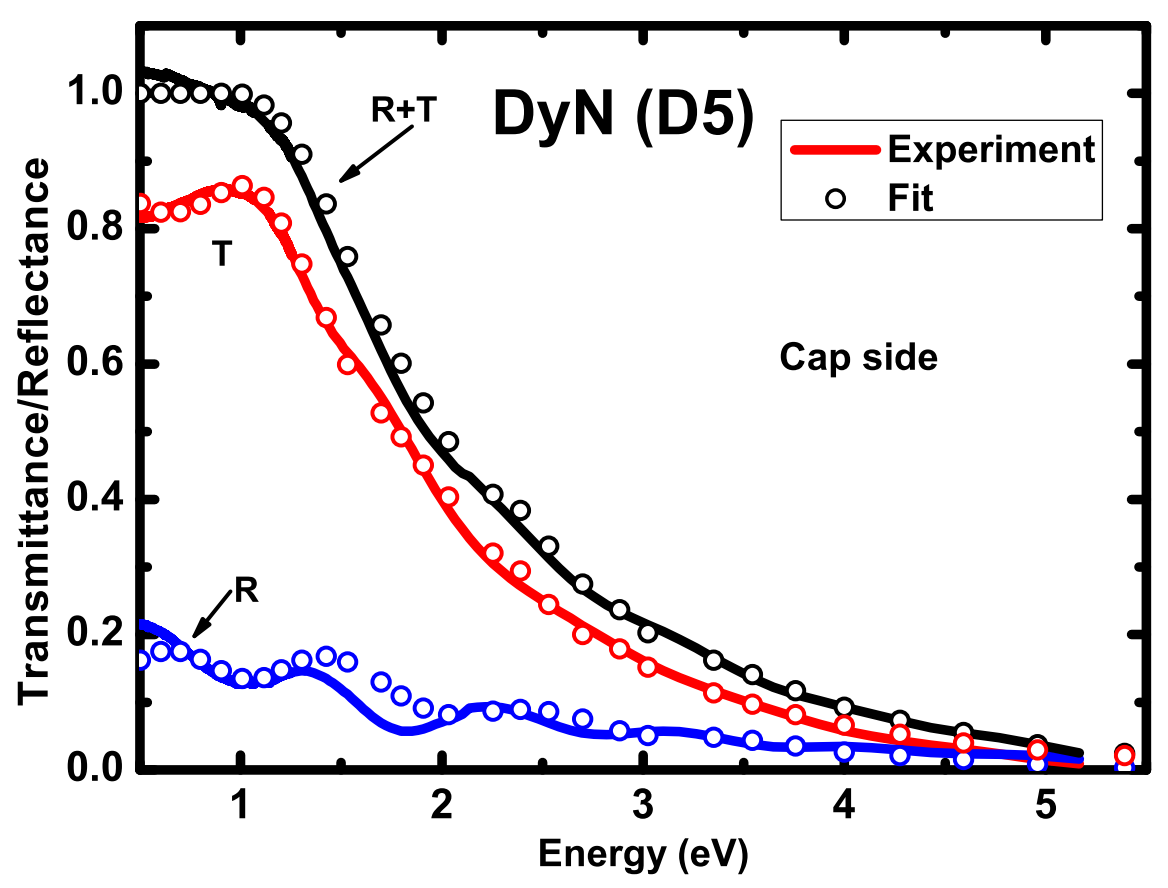

Figure 6.9 $R, T, R+T$ from the cap side of a $D y N(D 5)$ thin film. 
Figures 6.10 \& 6.11, illustrate optical spectra from film DyN, D3, of thickness $320 \mathrm{~nm}$ which was prepared at a considerably lower $\mathrm{N}_{2} / \mathrm{Dy} \&$ flux ratio as compared to the film D5. A significant difference is immediately noticeable at low energy in the shape of the transmittance curve compared to Fig. 6.8, particularly at $0.5 \mathrm{eV}$, where the transmittance is as low as $70 \%$ as opposed to $80 \%$ at $1.0 \mathrm{eV}$. This is the effect of a high carrier density, of the order of $10^{21} \mathrm{~cm}^{-3}$, introduced due to the nitrogen vacancies. An electron effective mass of $\mathrm{m}^{*}=0.2 \mathrm{~m}_{0}$, obtained from the band structure (Fig. 6.1) was used in the calculation of free carrier density in Eq. 4.3 and 4.4. 


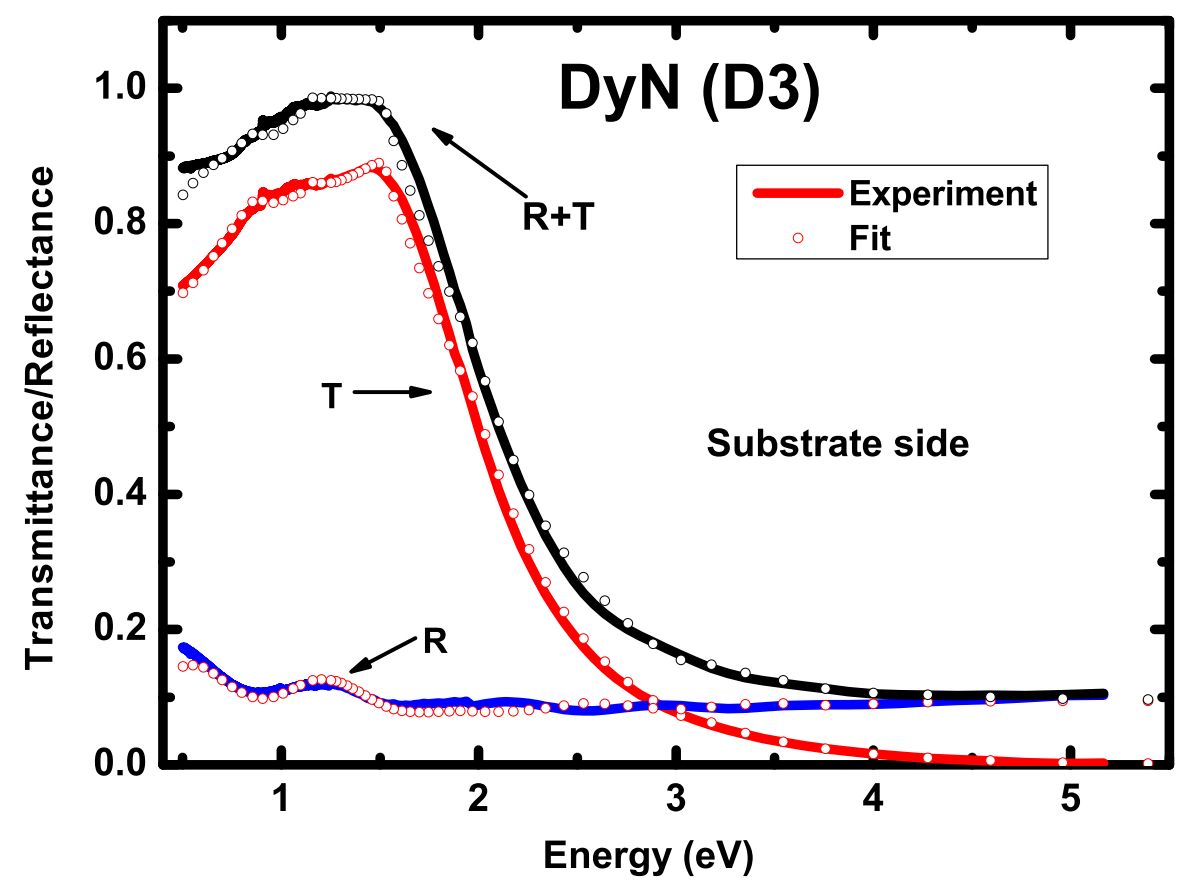

Figure 6.10 $R, T \& R+T$ spectra of a DyN (D3) film with nitrogen vacancies. The solid lines are experimental data and fitting is represented by open circles.

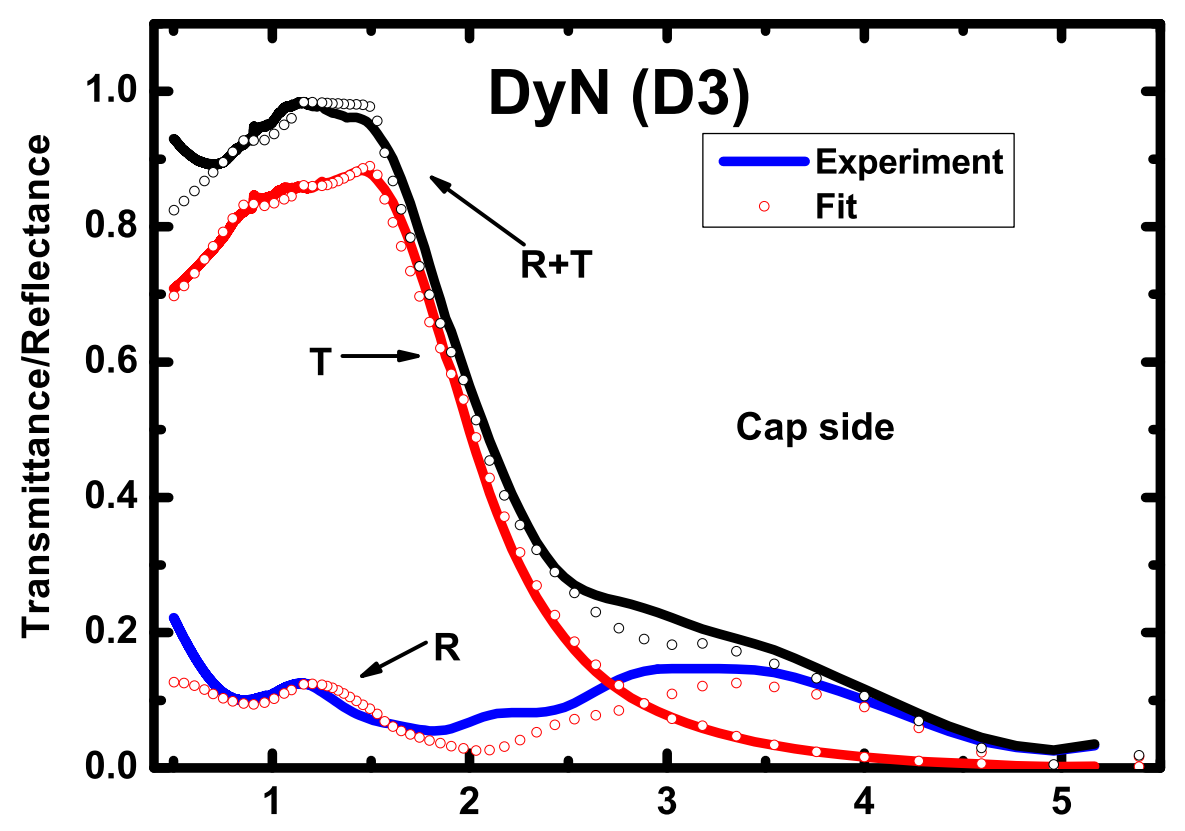

Figure 6.11 $R, T \& R+T$ from the cap side of $D y N(D 3)$ thin film. 
Returning to the near stoichiometric film, the extinction coefficient, determined by the fitting of the experimental optical spectra of thin film of DyN (D5) is shown in Fig. 6.12. An onset of the absorption is seen at nearly $1.2 \mathrm{eV}$ after which absorption increases monotonically. In the absence of any other structure in the extinction coefficient at higher energy, it is established that a near stoichiometric thin film of DyN has a direct energy gap of $1.2 \pm 0.05 \mathrm{eV}$ in its paramagnetic state. It agrees, within uncertainty, with the direct energy gap value ${ }^{2}$ of $1.17 \mathrm{eV}$ at the $\mathrm{X}$ - point predicted by Larson et al. (Fig. 6.1). Also note that in the region below the energy gap $k$ is nearly zero, evidence of a very small free carrier density in the film. In contrast, the absorption coefficients of the DyN films with greater nitrogen vacancies show significant absorption in the subgap region. However the effect of a higher density of free carriers does not end here. Fig. 6.13 clearly illustrates that the DyN thin film with larger nitrogen vacancy concentration shows a more prominent shift in the absorption edge, $1.7 \mathrm{eV}$ for the (D2) sample. Note that the film (D2) was prepared at a lower nitrogen partial pressure $\left(6.8 \times 10^{-5}\right.$ mbar $)$ while the deposition rate for the dysprosium metal was $2.0 \AA / \mathrm{s}$, the highest in the Table 6.1. Another sample of DyN, (D3) grown at a relatively larger nitrogen pressure of $1.7 \times 10^{-4}$ mbar and a deposition rate $1.5 \AA / s$ had a nitrogen to dysprosium ratio closer to the stoichiometric value showed a relatively smaller shift of the absorption coefficient. Table 6.2 summarises the effects of growth conditions on the fundamental absorption edges of various DyN thin films.

Table 6.2 Effect of Nitrogen Vacancies on the Fundamental Absorption Edge

\begin{tabular}{|c|c|c|c|c|c|}
\hline & $\begin{array}{c}\mathrm{N}_{2} / \text { Dy } \\
\text { Flux Ratio }\end{array}$ & $\begin{array}{c}\alpha \\
\text { at } 0.5 \mathrm{eV} \\
\left(10^{3} \mathrm{~cm}^{-1}\right)\end{array}$ & $\begin{array}{c}\text { Absorption } \\
\text { Onset } \\
(\mathrm{eV})\end{array}$ & $\begin{array}{c}\text { Moss-Burstein } \\
\text { Shift } \\
(\mathrm{eV})\end{array}$ & $\begin{array}{c}\text { DyN } \\
\text { Thickness } \\
(\mathrm{nm})\end{array}$ \\
\hline \hline D5 & 250 & 0 & 1.2 & & 287 \\
\hline D3 & 75 & 6.5 & 1.5 & 0.3 & 304 \\
\hline D2 & 22 & 9.7 & 1.7 & 0.5 & 320 \\
\hline
\end{tabular}




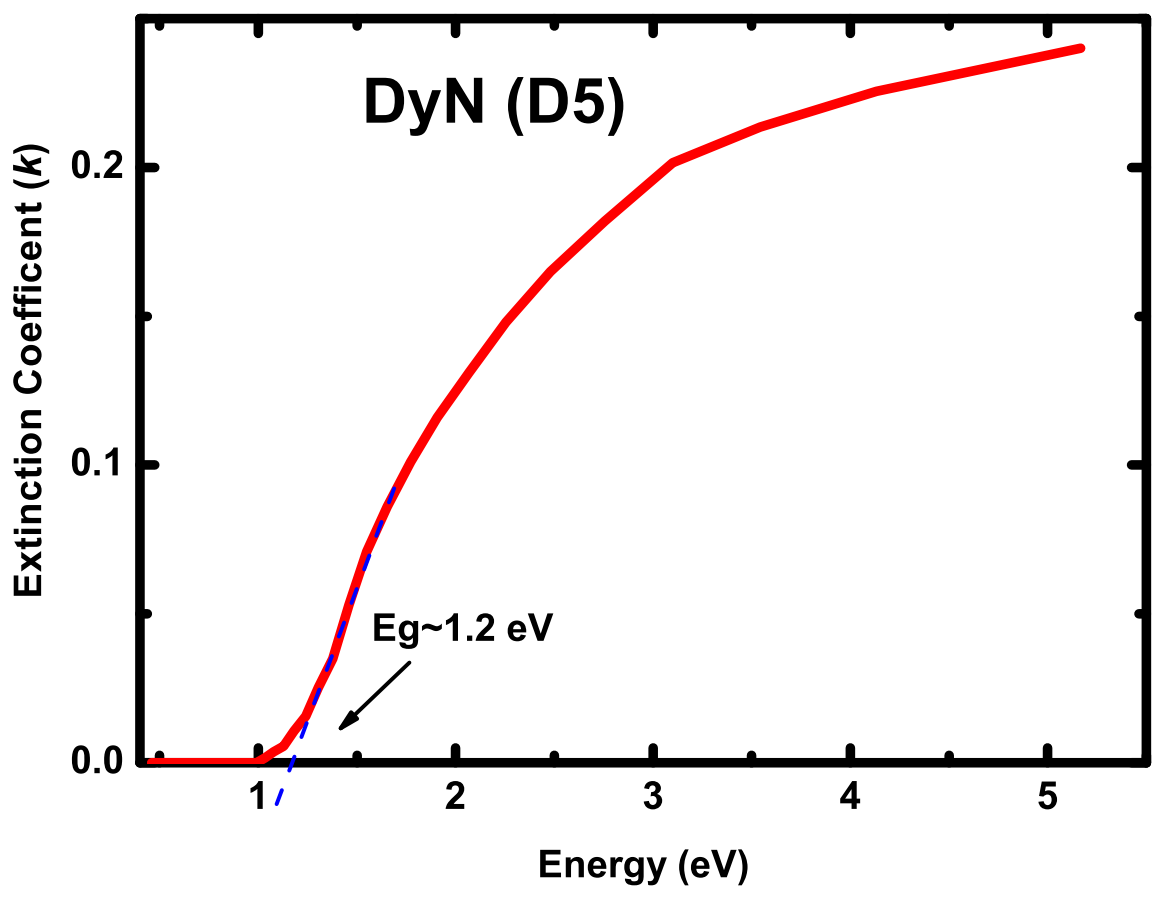

Figure 6.12 Imaginary part of the complex refractive index for a near stoichiometric DyN(D5) film against photon energy showing the onset of absorption at around $1.2 \pm 0.05 \mathrm{eV}$.

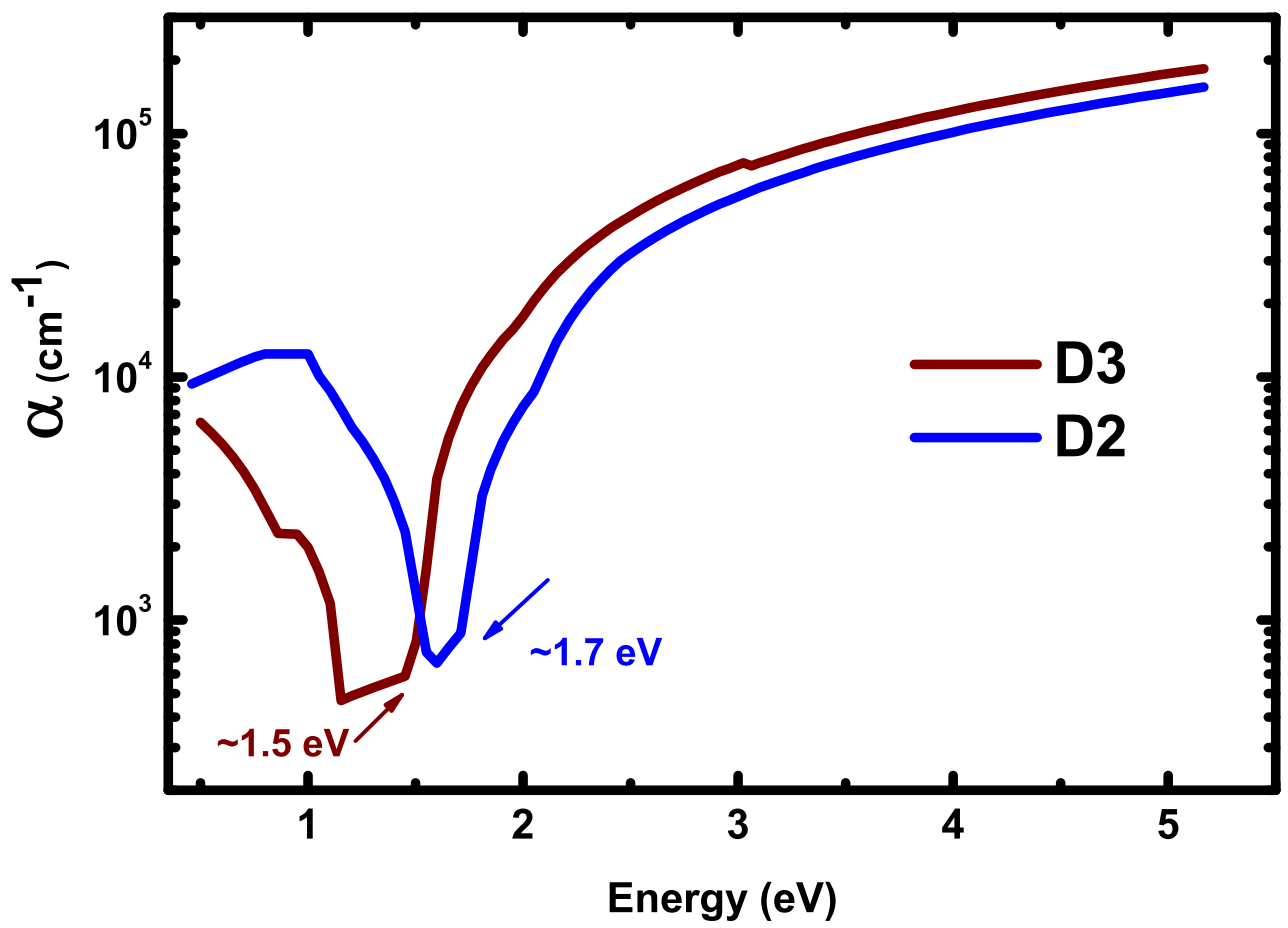

Figure 6.13 Absorption coefficient for DyN samples with significant levels of nitrogen vacancies. 
Figure 6.14, further illustrates the relation between the free carriers and the band gap with $\mathrm{N}_{2}$ /Dy flux ratio during the growth. Those carriers are accommodated in the three electron pockets at the Fermi surface in $\mathbf{k}$-space, and then may introduce a degenerate electron gas of Fermi energy $0.2 \mathrm{eV}$ to $0.3 \mathrm{eV}$ at X, in agreement with the Moss-Burstein shift of the absorption edge seen in Fig. 6.13. The Moss-Burstein effect results from the Pauli exclusion principle and is seen in semiconductors as a shift with the increasing doping of the absorption edge. The shift arises because the Fermi energy $\left(E_{F}\right)$ lies in the conduction band for heavy n-type doping (or in the valence band for p-type doping). The filled states therefore block thermal or optical excitation. Consequently the measured band gap determined from the onset of interband absorption moves to higher energy (i.e. suffers "a blue shift"). It is now known that the higher concentration of nitrogen vacancies donate more conduction electrons ${ }^{89}$, pushing the Fermi level into the conduction band. An electron, in the case of a degenerate semiconductor, can only be excited into conduction band, above the Fermi level, if there is an available state according to Pauli's exclusion principle.

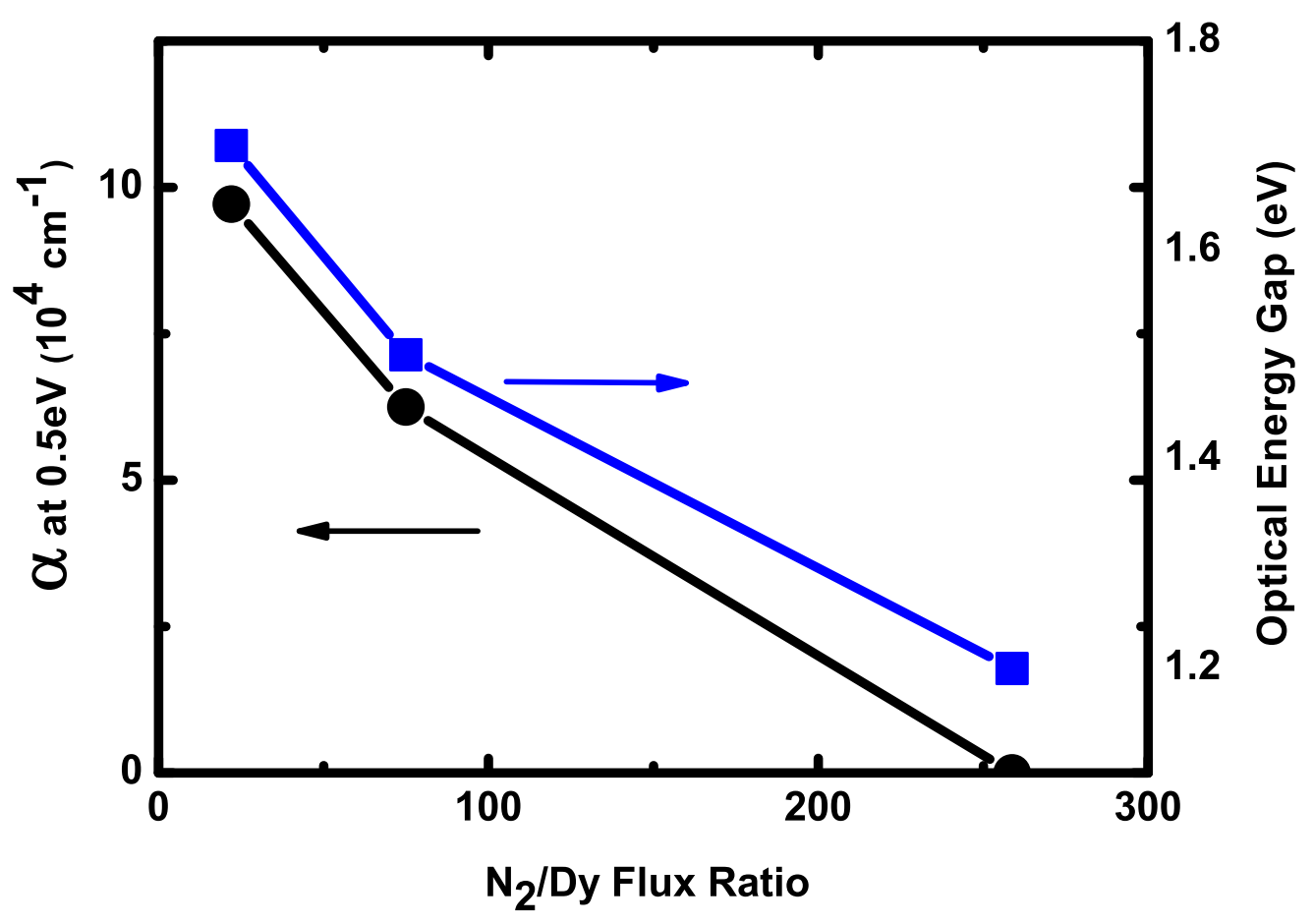

Figure 6.14 Free carrier absorption (black solid circles) and the optical gap (blue solid squares) vs. $\mathrm{N}_{2} /$ Dy flux ratio during growth. 
The real and imaginary parts of the dielectric function are shown in Fig. 6.15 for the near stoichiometric film, D5. Considering the imaginary part, $\varepsilon^{\prime \prime}$, first, the absorption increases monotonically with the energy, showing no structure that might result from interband onsets at any energy above the first optical absorption edge. The rapid drop near the edge extrapolates to a gap of about $1.2 \pm 0.05 \mathrm{eV}$, with a tail to lower energy that we believe is related to uncertainties in the parameters due to incomplete correction for the interference fringes. These first ever experimental observations of the direct energy gap as well as the dielectric functions are in disagreement with the theoretically predicted values. The theoretical dielectric constant ${ }^{167,168}$ of DyN is reported as 6.55 or 6.57 . Since the band structure of DyN is quite similar to $\mathrm{GdN}$, these experimental observation could be compared with the dielectric function of GdN as calculated by Mitra et al. ${ }^{120}$ The refractive index value deduced in their work is around 3.3 for GdN which again is in disagreement with the experimentally determined $\mathrm{GdN}$ value of $2.0 \pm 0.1$ of the present work.

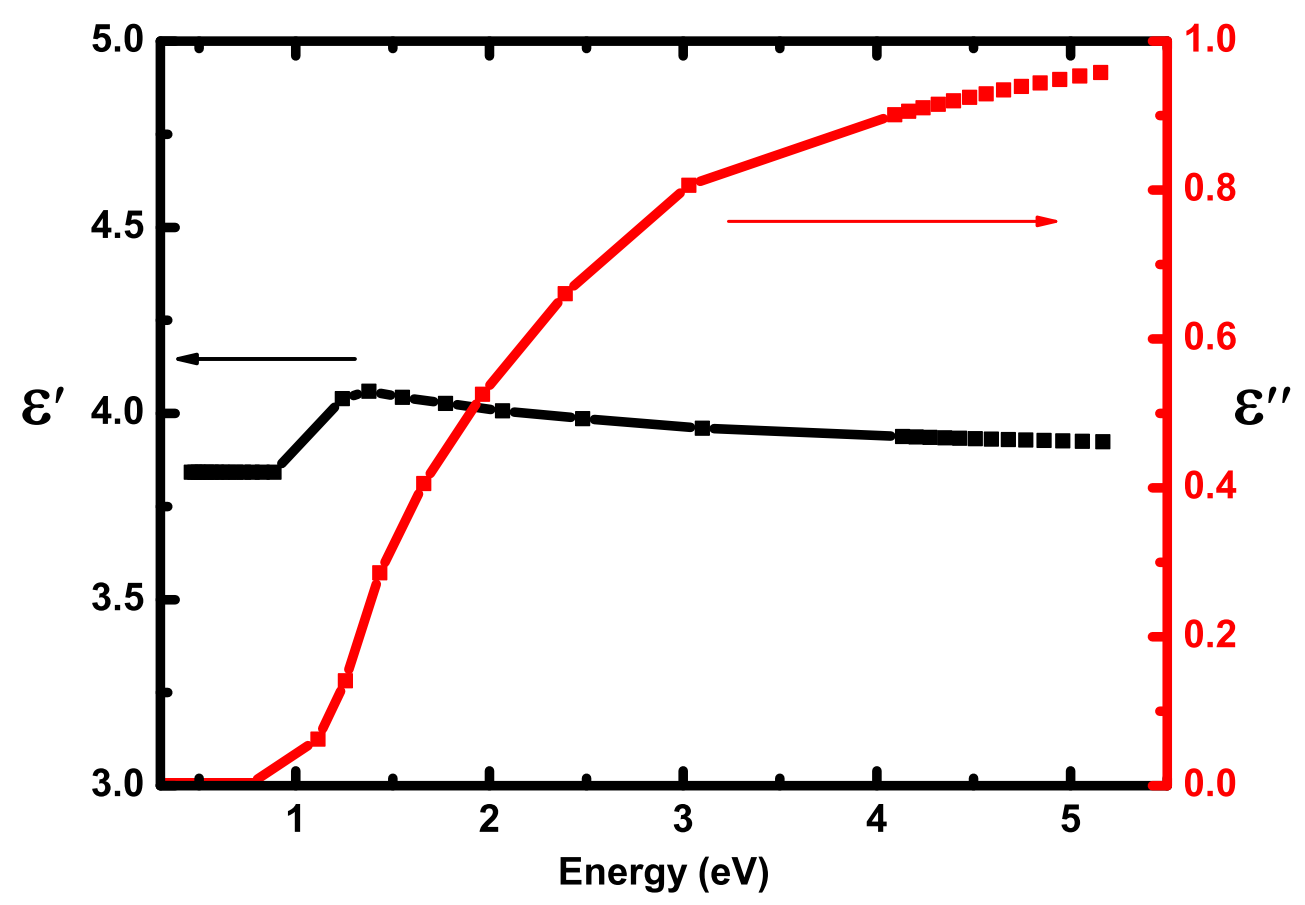

Figure 6.15 Real and Imaginary parts of the dielectric function. 


\subsection{Optical Spectra in the Far Infrared Region}

Optical spectra for DyN thin films in the far infrared region were collected and analysed by our collaborators, Veronica Goian and Stanislav Kamba, at the Institute of Physics, Academy of Sciences of the Czech Republic. For this analysis samples were prepared on YSZ substrates at the room temperature with a silicon capping layers by Harry Warring.

Figure. 6.16, far infra red reflectivity of the bare YSZ substrate and of the $\mathrm{Si}$ - capped DyN film on the YSZ substrate. These data can be fitted with a model that includes only two damped oscillators; the dominant $\mathrm{TO}$ phonon expected in the $\mathrm{NaCl}$ structure is here at $280 \mathrm{~cm}^{-1}(0.035 \mathrm{eV})$, damping constant $160 \mathrm{~cm}^{-1}$; the frequency is somewhat lower than the estimated $338 \mathrm{~cm}^{-1}(0.042 \mathrm{eV})$ based on an LSDA $+U$ approximation. ${ }^{187}$ The mode gives a contribution of 20 to the de dielectric constant (Fig. 6.17). A satisfactory fit required also a weaker resonance at $1200 \mathrm{~cm}^{-1}(0.15 \mathrm{eV})$, damping $2400 \mathrm{~cm}^{-1}$ and the dielectric contribution of 1.8. We assign this to a transition from nitrogen vacancy states expected to lie close below the conduction band. ${ }^{199}$ The fit also returns a high-frequency dielectric constant of 4.4, in reasonable agreement with the near IR dielectric function of $4.0 \pm 0.1$. 


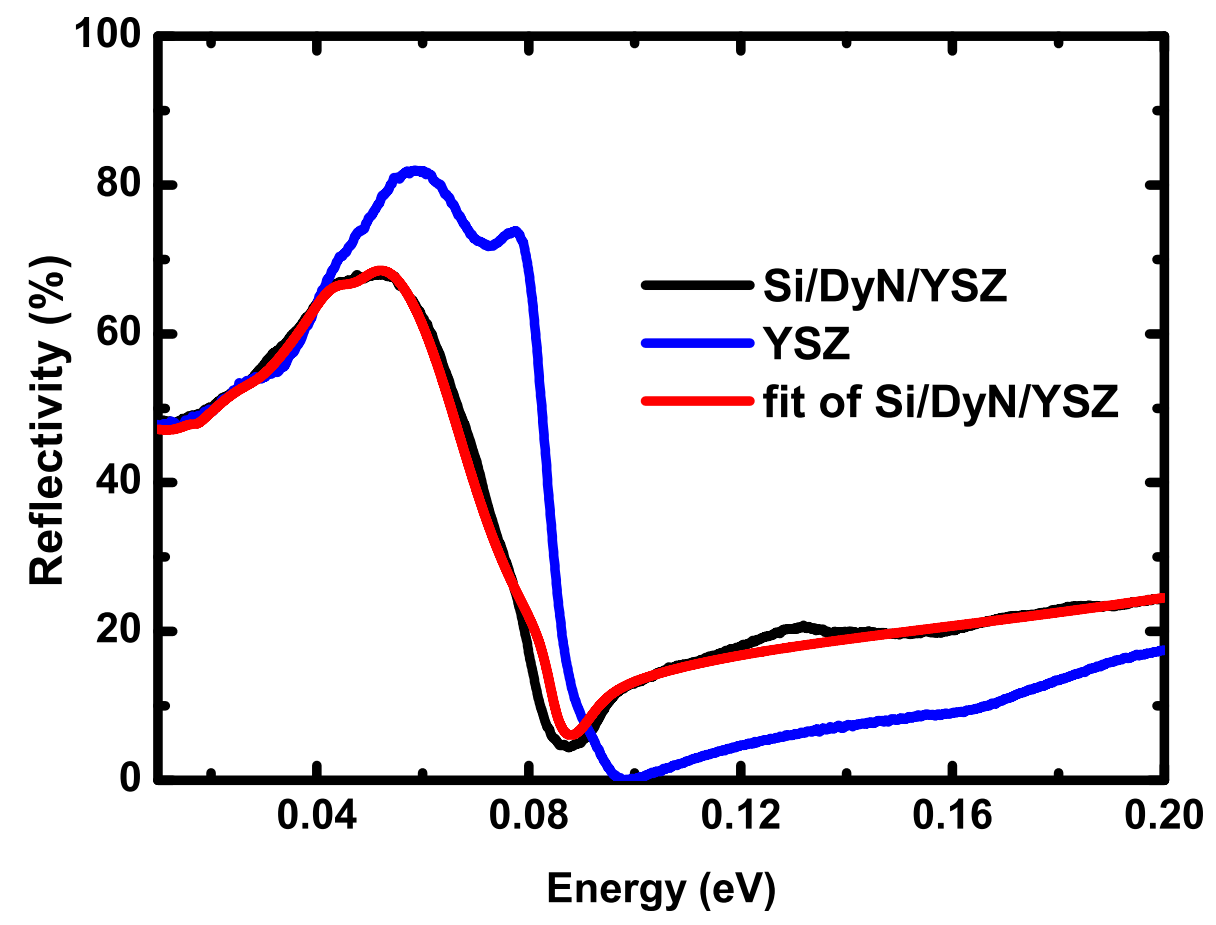

Figure 6.16 Infrared reflectivity spectrum of a $\mathrm{Y}$-stabilized $\mathrm{ZrO}_{2}$ substrate and a DyN thin film on a YSZ substrate capped by amorphous Si along with a fit to the .

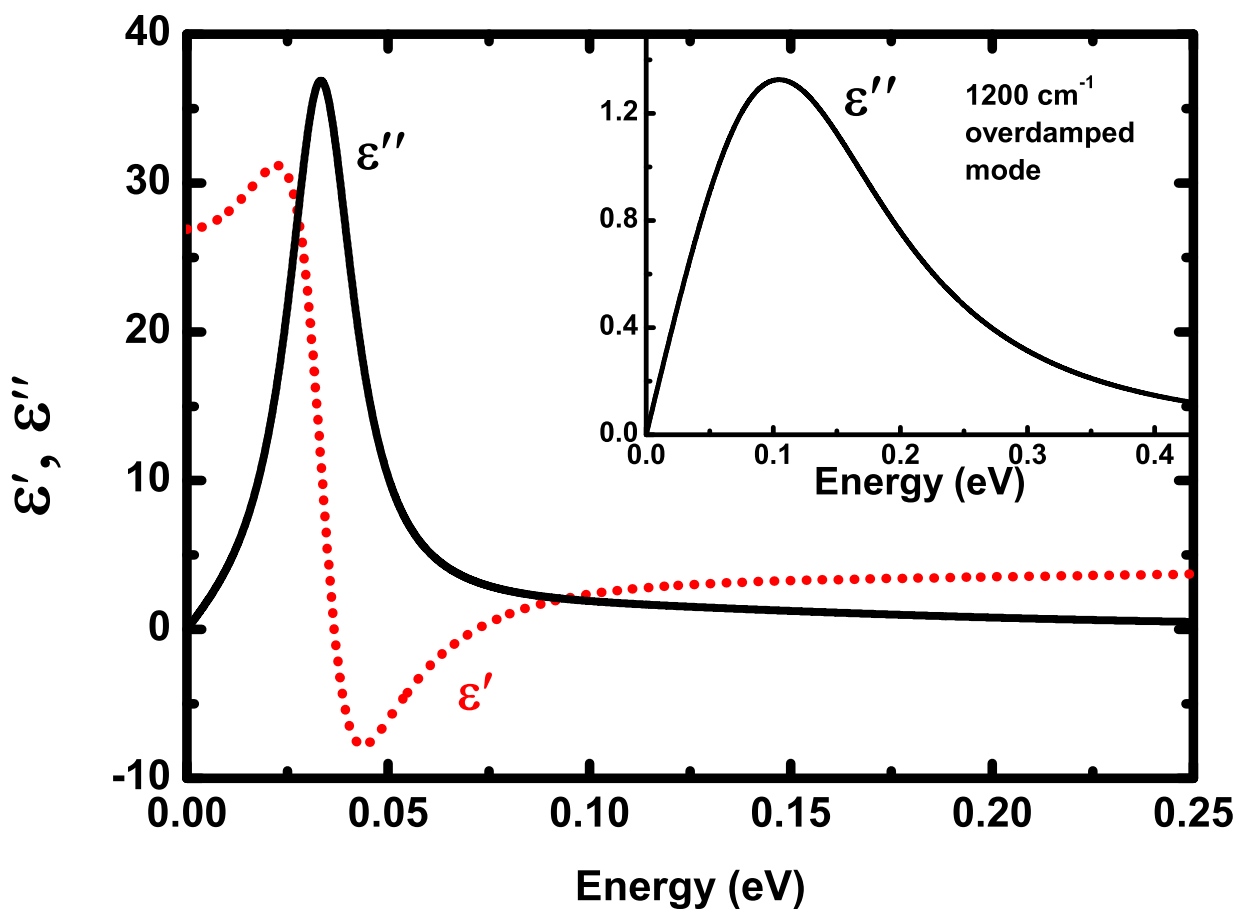

Figure 6.17 Fitted real and imaginary parts of the complex dielectric function of DyN showing the polar phonon and nitrogen - vacancy donor to conduction band transition. 


\subsection{Temperature Dependence of Transmission Spectra}

Since a ferromagnetic phase below $20 \mathrm{~K}$ in thin films of DyN was observed (Fig. 6.4) therefore it was expected to show a red - shift in its fundamental absorption edge like GdN. But the experimental transmittance spectra do not show any systematic temperature dependence at all as shown in Fig. 6.18. The sample temperature might have risen during the measurements when light falls on it. Perhaps measurements at temperatures lower than the $5 \mathrm{~K}$ would have shown some shift in the optical energy gap. Our system, however, was not capable of performing measurements below $5 \mathrm{~K}$.

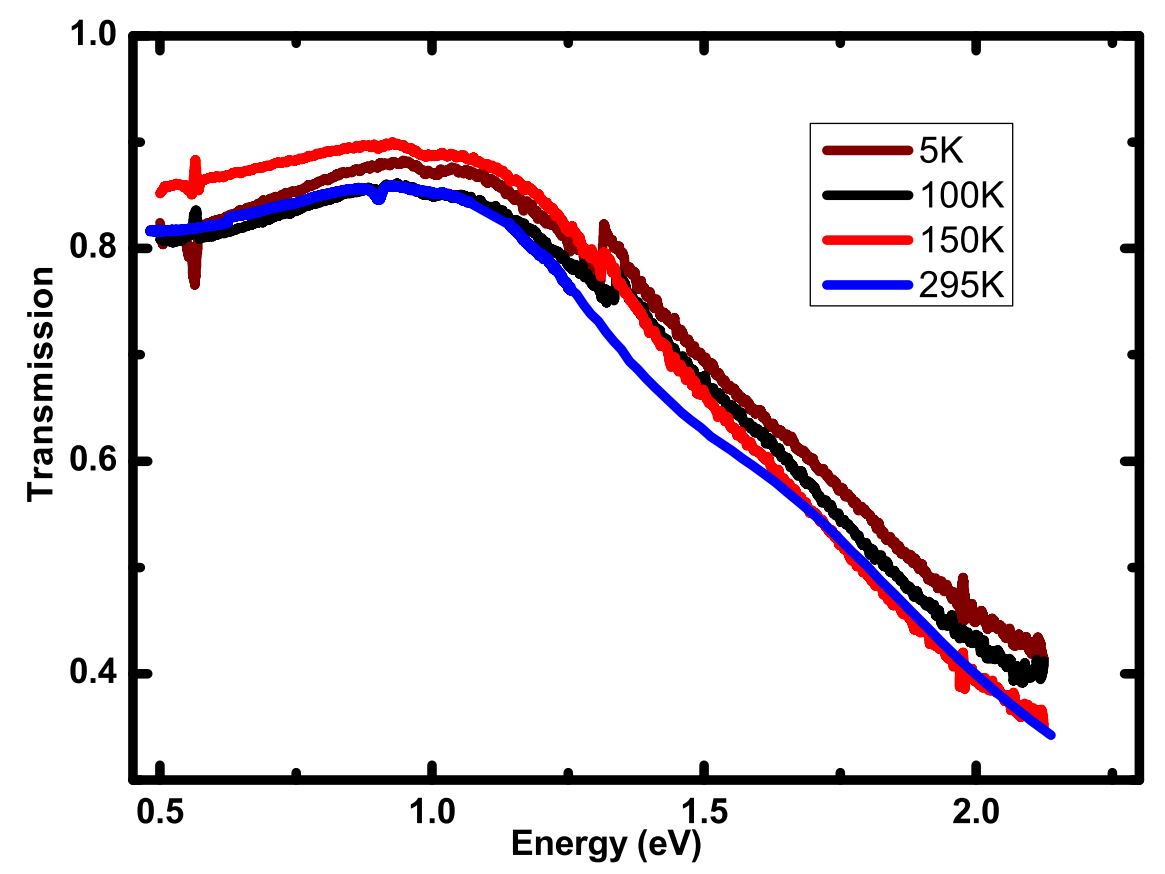

Figure 6.18 Temperature dependence of the absorption edge in DyN(D5) thin films. 


\subsection{Conclusion}

The electronic structure of thin films of DyN was investigated for the first time experimentally. Magnetic measurements confirmed the presence of a ferromagnetic phase below $20 \mathrm{~K}$. The semiconducting nature of DyN was confirmed by the resistivity measurements. Optical transmission and reflection spectra at room temperature show the presence of an optical energy gap of $1.2 \pm 0.05 \mathrm{eV}$ in agreement with the theoretical value of $1.17 \mathrm{eV}$ within uncertainty. The refractive index of the film is calculated to be $2.0 \pm 0.1$ which was further corroborated by the far infrared reflectivity measurements, however there are no other experimental reports to compare with this value. 


\section{Chapter 7}

\section{Samarium Nitride and Europium Nitride}

Due to their proximity in the periodic table, the light rare earth metals, samarium and europium, are of particular interest because of their antiparallel $\mathrm{L}$ and $\mathrm{S}$ moments according to Hund's rule. On the optical energy gaps, there are a few reports for SmN and EuN. The energy gap as obtained from X-ray absorption and emission (XAS/XES) spectra ${ }^{147}$ of $\mathrm{SmN}$ thin films is $1.5 \mathrm{eV}$, noticeably larger than the $0.70 \mathrm{eV}$ of bulk $\mathrm{SmN}$ crystals. ${ }^{80,83,200}$ The theoretically ${ }^{2}$ estimated direct gap at the $\mathrm{X}$ point is $0.81 \mathrm{eV}$ and an indirect gap at $\Gamma-\mathrm{X}$ is $0.48 \mathrm{eV}$ when $\mathrm{T}>\mathrm{T}_{C}$. Similarly for EuN, a disagreement between the theoretical and experimental values of the energy gap is found. Experimental values $^{3,80,83,107,200}$ of $\mathrm{E}_{g}=0.76 \mathrm{eV}$ and $0.90 \mathrm{eV}$ are appreciably different from the theoretical value ${ }^{2}$ of $1.20 \mathrm{eV}$.

Optical energy gaps for the polycrystalline $\mathrm{SmN}$ and $\mathrm{EuN}$ thin films are being reported for the first time by reflection/transmission spectra in this chapter. Note that the magnetic measurements were not available for these samples.

\subsection{Growth of SmN and EuN thin films}

Metallic ingots (99.9\% pure) of Sm and Eu were stored in mineral oil and were cleaned with ethanol before putting them in the growth chamber in tantalum boats. A thin film of $\mathrm{SmN}$ was grown thermally at a base pressure of $8.4 \times 10^{-9}$ mbar. The average growth pressure was of the order $10^{-4}$ mbar where the average growth rate of the film was $0.4 \AA / \mathrm{s}$. The $\mathrm{SmN}$ film was grown on a sapphire substrate and was capped with a $\mathrm{MgF}_{2}$ layer to protect it from oxidation and debris.

Growth of EuN took place under the base pressure of $7.8 \times 10^{-8}$ mbar on a YSZ substrate with a AlN capping layer. The nitrogen partial pressure was around $2.0 \times 10^{-4}$ mbar with a deposition rate of $1.0 \AA$ /s. Eu metal was evaporated from thermal source (Ta boats) with 
$\mathrm{N}_{2}$ gas supplied via an ion source producing $128 \mathrm{eV} \mathrm{N}^{2+}$ ions at a discharge current of 0.32 A. The film was grown by Dr. Jan Richter.

\subsection{Structural Analysis}

Figure 7.1 shows the XRD pattern of a $\mathrm{SmN}$ thin film. After the sapphire, the $\mathrm{SmN}$ phase is the most prominent. At around $2 \theta=30.8$, we see a peak at (111) followed by rather weak peaks from (220) and (311) indicating the preferred orientation alon $<111>$ direction. The lattice constant is approximately $0.502 \pm 0.003 \mathrm{~nm}$ and the average crystallite size comes out to be larger than $20 \mathrm{~nm}$, considerably bigger than the crystallite sizes of GdN and DyN films reported in this work.

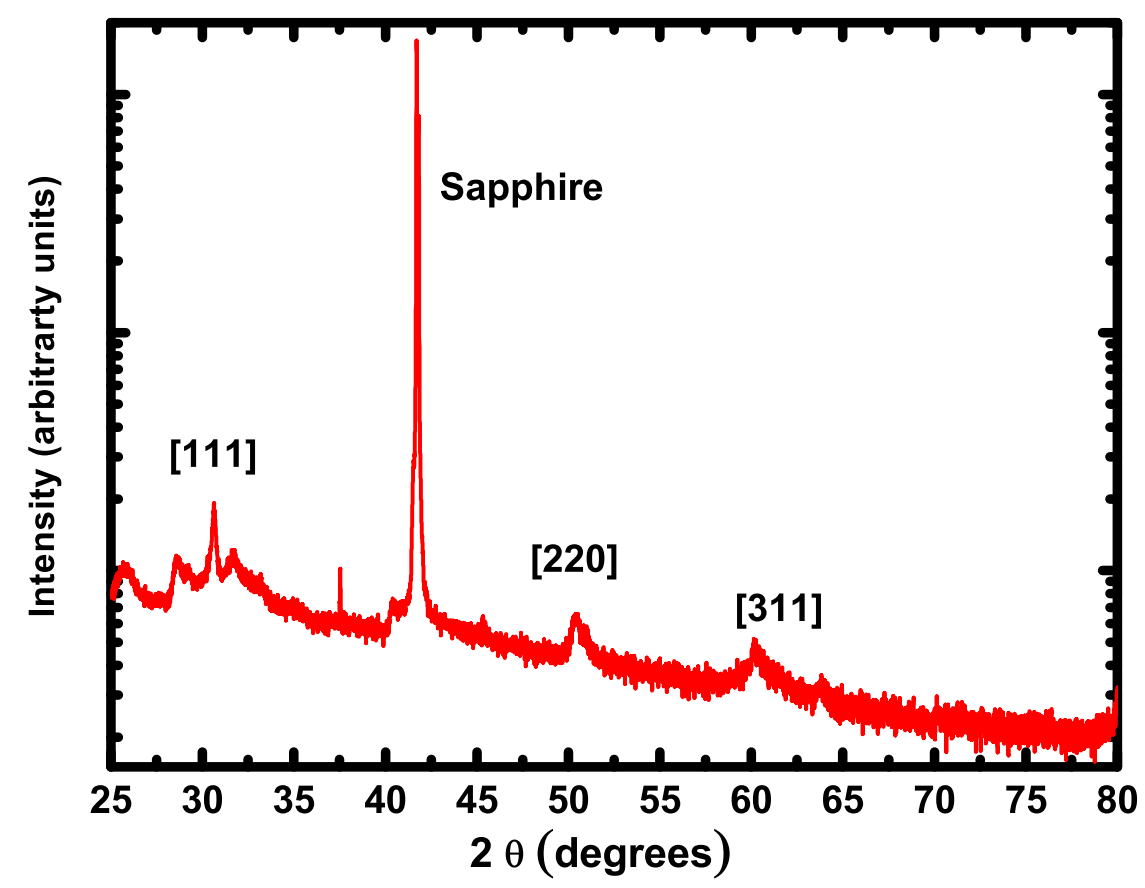

Figure 7.1 XRD spectrum for a SmN thin film. 


\subsection{Optical Spectra of SmN}

Figure 7.2 shows the reflectance $(\mathrm{R})$, transmittance $(\mathrm{T})$ and sum of both $(\mathrm{R}+\mathrm{T})$ from the cap side of the SmN thin film measured from around $280 \mathrm{~nm}$ thick $\mathrm{SmN}$ at room temperature. Value of film thickness was confirmed by the RBS measurements. Apart from interference, slightly more than $65 \%$ average transmittance is clearly visible in the energy region of $0.5 \mathrm{eV}$ to $1.0 \mathrm{eV}$, nearly $20 \%$ lower than the reported transmittance for GdN and DyN in the same energy region. This effect was associated with the free carrier absorption in the subgap region. A density of free carriers greater than $10^{20} / \mathrm{cm}^{3}$ was estimated by using equations $4.2 \& 4.4$, where $\mathrm{m}^{*}=0.20 \mathrm{~m}_{0}$ is the effective mass calculated from the band structure ${ }^{2}$ of $\mathrm{SmN}$ and $\sigma_{D C}$ was used from the resistivity data of Preston et al. ${ }^{147}$ The average reflectance in the subgap energy range is around $10 \%$ as expected making the sum of reflectance and transmittance $(\mathrm{R}+\mathrm{T})$ close to $80 \%$. Above $1.0 \mathrm{eV}$, the transmission of light starts falling gradually till $5.5 \mathrm{eV}$ indicating interband electronic transitions.

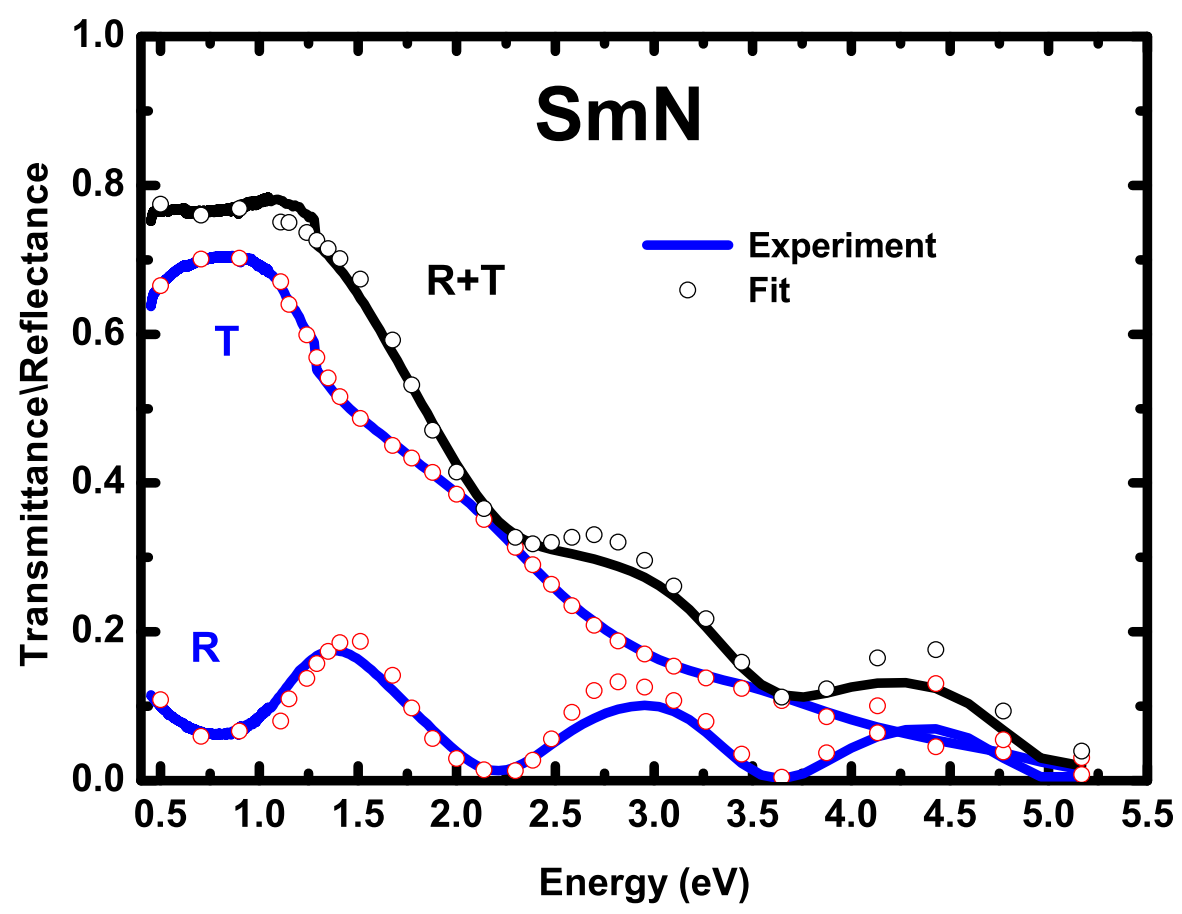

Figure 7.2 Reflectance $(R)$, Transmittance $(T)$ and their sum $(R+T)$ measured from a SmN film. 
Open circles in Fig. 7.2 represent simulated reflectance and transmittance spectra. Note that due to strong effects of free carrier absorption in the sub gap region, an estimate of the refractive index was difficult. However, guided by the results for $\mathrm{GdN}$ and DyN described in previous chapters, the refractive index for $\mathrm{SmN}$ should not be very different from the $2.0 \pm 0.1$. Figure 7.3 shows the imaginary part of the complex refractive index as obtained by fitting the experimental spectra with a refractive index of 2.1. As was also clear from $\mathrm{R}$ and $\mathrm{T}$ spectra, a high density of free carriers is visible in the low energy region of the $k$ spectrum. An onset of absorption due to direct interband optical electronic transitions at the point $\mathrm{X}$ is found at $1.3 \mathrm{eV}$ which is the same as reported for $\mathrm{GdN}$ but larger than for DyN. However due to the large electron concentration in the SmN sample, a blue shift in the onset of absorption may be present as is seen for the case of DyN with greater nitrogen vacancies. ${ }^{100}$ Thus an uncertainty in the energy gap value might be as much as $0.1 \mathrm{eV}$. The calculated absorption coefficient (shown inset) is higher than $10^{4} / \mathrm{cm}$. In the absence of Drude characteristics, the optical data seem to show that $\mathrm{SmN}$ has a semiconducting band gap.

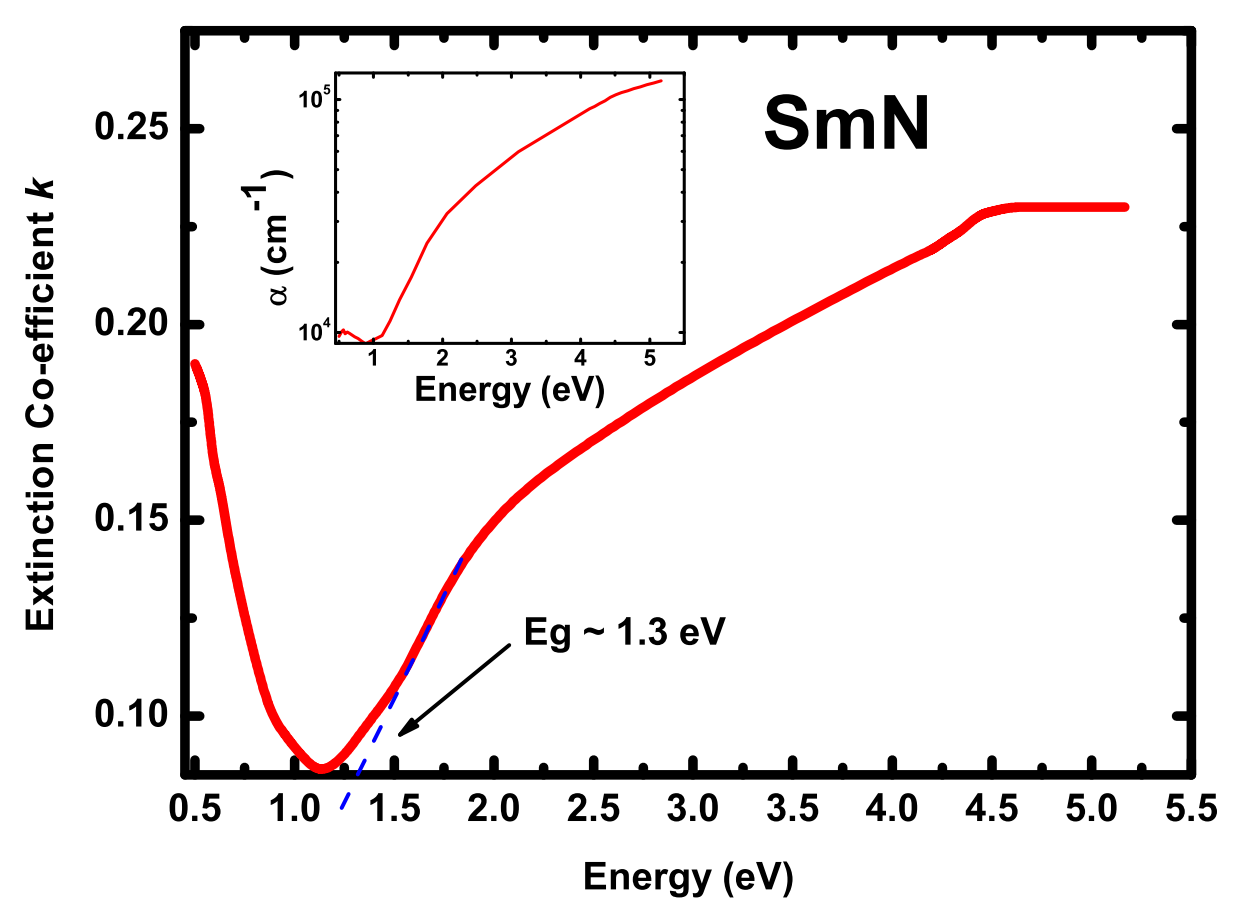

Figure 7.3 Plot of extinction coefficient versus energy for $S m N$. Absorption coefficient (inset) rises above $10^{4} / \mathrm{cm}$.

The value of optical energy gap derived from the extinction coefficient lies almost in the 
middle of previous experimental optical energy gap values ${ }^{80,83,200}$ of $0.70 \mathrm{eV}$ and $1.5 \mathrm{eV}$ measured at room temperature. ${ }^{147}$ The reported value is also larger than the theoretical ${ }^{2}$ onset of direct absorption at $0.81 \mathrm{eV}$.

\subsection{Optical Spectra for EuN}

Figure 7.4 shows the reflectance $(\mathrm{R})$, transmittance $(\mathrm{T})$ and their sum, $\mathrm{R}+\mathrm{T}$ spectra collected at room temperature as a function of photon energy range $0.2 \mathrm{eV}$ to $1.6 \mathrm{eV}$ collected at room temperature. The film shows the lowest transmission of light in the subgap region compared to all other samples reported in this work. A less than $50 \%$ transmission in the region of photon energy below $0.8 \mathrm{eV}$ is clearly an effect due to strong carrier concentrations of electrons estimated to be higher than $10^{20} / \mathrm{cm}^{3}$. Therefore fitting of the transmission and reflection spectra was not viable to determine the complex refractive index. The absorption coefficient (Fig. 7.5) was estimated by using the equation $\mathrm{T}=(1-\mathrm{R}) \exp (-\alpha \times \mathrm{d})$ where $\mathrm{R}$ and $\mathrm{T}$ are the reflection and the transmission coefficients respectively, "d" is the thickness and $\alpha$ is the absorption coefficient. Note that this equation does not take interference effects into account. The resulting value of the absorption onset for EuN thin film is $0.97 \pm 0.05 \mathrm{eV}$. Interestingly this value of energy gap, like the energy gap in $\mathrm{SmN}$, also lies between previously reported experimental ${ }^{83,107}$ value of $0.76 \mathrm{eV}$ and theoretical ${ }^{2}$ value of $1.20 \mathrm{eV}$ however note that the energy gap value for EuN might be slightly overestimated due to the presence of free carriers. 


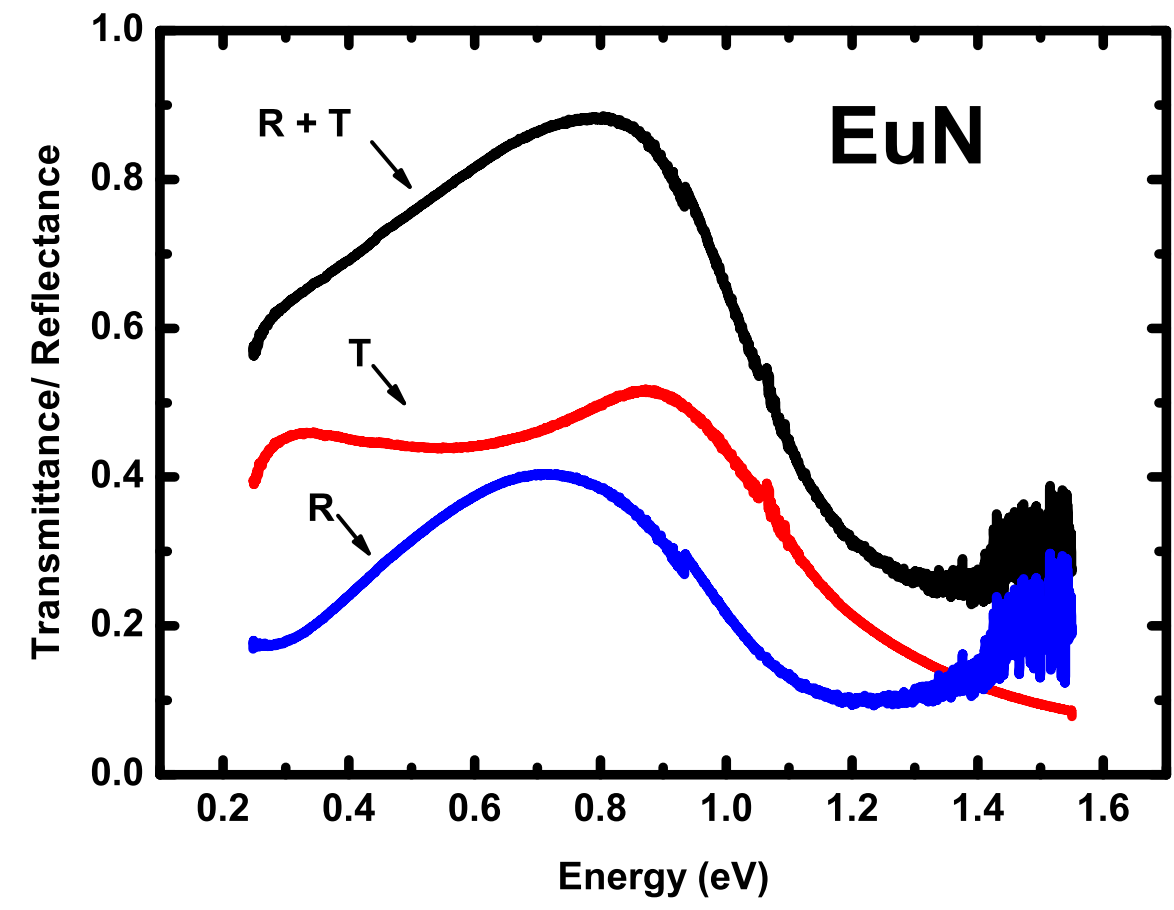

Figure 7.4 Reflectance and transmittance and their sum for thin film of EuN in the energy.

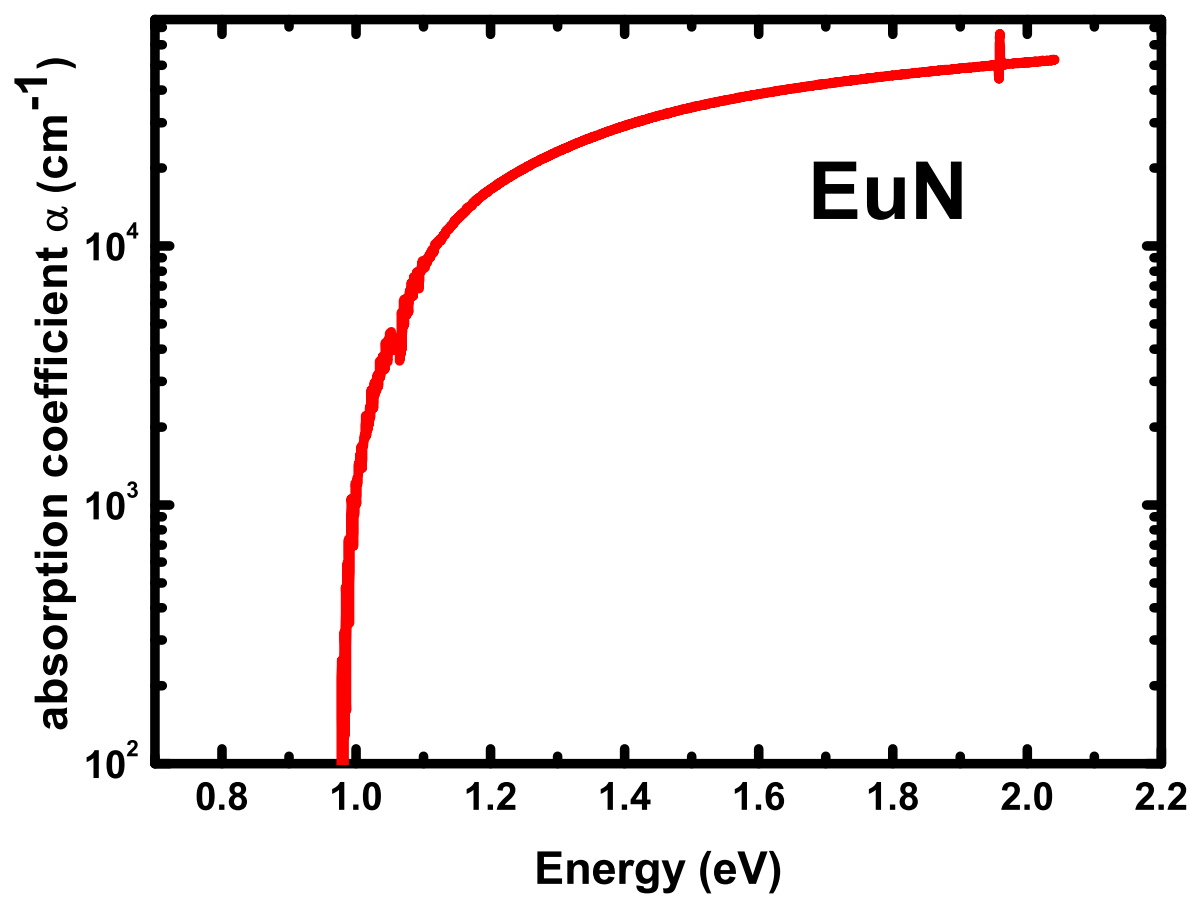

Figure 7.5 Energy dependent absorption coefficient for a EuN thin film estimated from the room temperature transmission spectrum indicating a fundamental absorption edge around $0.97 \pm 0.05 \mathrm{eV}$. 
The presence of a finite energy gap does not necessarily suggests that EuN is a semiconductor in its ground state. However a band structure calculation for EuN by quasiparticle self - consistent GW (QSGW) and tuned to the experimentally determined energy gap of $0.97 \pm 0.05 \mathrm{eV}$ of this work in fact returns a ferromagnetic semiconducting solution for EuN (Fig. 7.6).The QSGW is based on self consistent perturbation theory where perturbation is minimized by self - consistency. ${ }^{201}$ A direct energy gap at X of $0.94 \mathrm{eV}$ in the QSGW band structure of EuN can be seen in Fig. 7.6. The calculated band structure also predicts an indirect gap between $\Gamma$ and $\mathrm{X}$ of about $0.31 \mathrm{eV}$. Note that the band structure of EuN exhibits a majority spin direct gap at $\mathrm{X}$ which is quite similar in energy to the measured optical absorption edge. It has been shown that in a paramagnetic state, an optical absorption edge can reasonably be estimated by taking the average of spin - up and spin - down states ${ }^{1}$ however the spin averaged gap for EuN is around $1.59 \mathrm{eV}$ which is higher than the ferromagnetic gap and the optical absorption onset because QSGW theory is known to provide an overestimate of the band gaps in semiconductors.

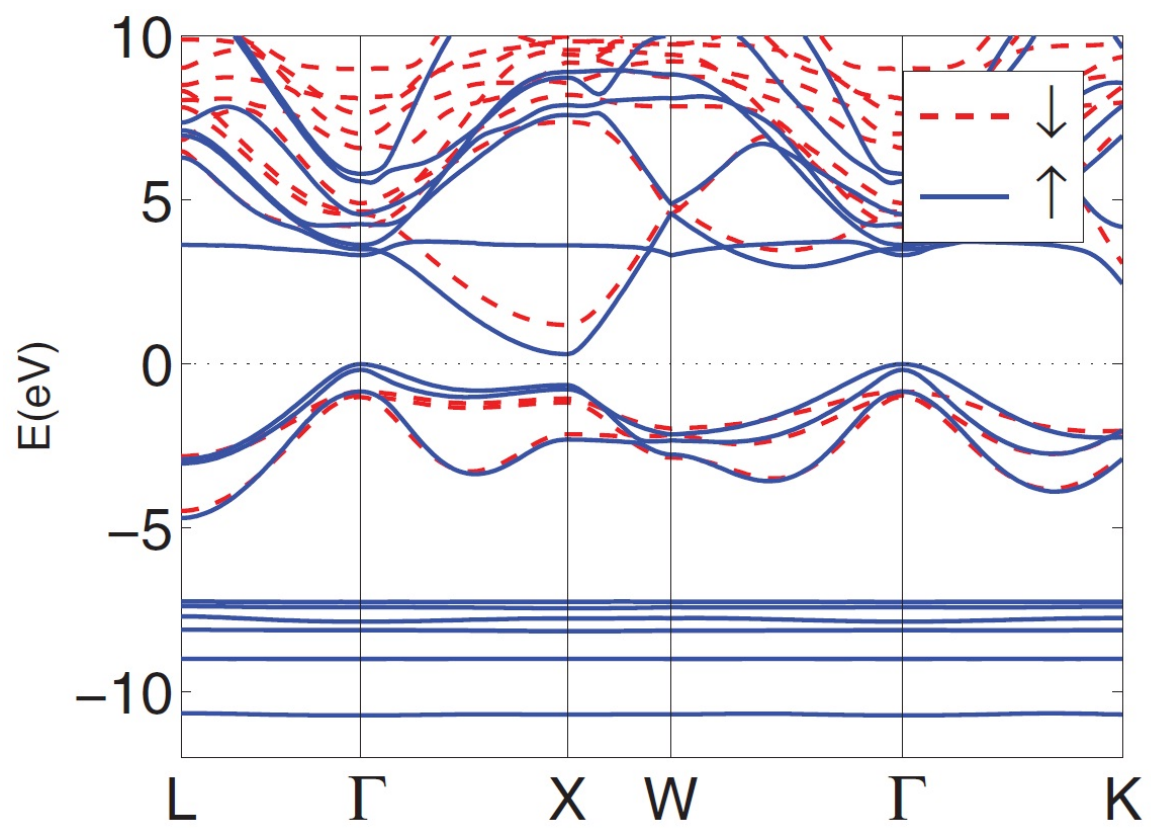

Figure 7.6 A ferromagnetic semiconducting band structure for EuN calculated by QSGW theory by Richter et al. ${ }^{3}$ 


\subsection{Conclusion}

The direct energy gap for $\mathrm{SmN}$ and $\mathrm{EuN}$ is determined for the first time by transmittance and reflectance spectra for polycrystalline thin films at room temperature. SmN shows a direct optical energy gap at $1.3 \pm 0.1 \mathrm{eV}$ and for EuN the energy gap value is $0.97 \pm 0.05 \mathrm{eV}$. Both samples show large effects due to free carriers in the subgap energy region. The fundamental absorption edge for $\mathrm{SmN}$ is not in good agreement with the previous results. A similar disagreement is also found for the energy gap in EuN. A QSGW band structure calculation engineered to the experimentally determined energy gap value of $0.97 \pm 0.05 \mathrm{eV}$, predict a semiconducting ground state for EuN. 


\section{Chapter 8}

\section{Final Discourse}

Despite considerable theoretical attention, there is a little evidence based on the sound experimentation to support the calculated electronic band structures of the rare earth nitride compounds. The major objective of this experimental thesis was to uncover the fundamental optical features of the rare earth nitride thin films and relate them to their electronic structures.

Stoichiometric polycrystalline thin films of GdN, DyN, SmN and $\mathrm{EuN}$ were prepared in an ultra high vacuum environment to aid the experimentation. It was determined that near stoichiometric films could be grown with the background nitrogen pressure of the order $10^{-4}$ mbar. A lower nitrogen partial pressure would result in the samples with nitrogen vacancies. Such films would have high carrier density showing subgap optical absorption in the transmission spectra. A capping layer of $\mathrm{MgF}_{2}$ proved to be very effective to protect the films from atmosphere and debris and to perform ex - situ the optical measurements at room temperature as well as at low temperature.

XRD measurements showed that the films were strongly textured along the $<111>$ direction, though weak signals from (222) planes were also visible. RBS measurements provided the atomic concentrations $/ \mathrm{cm}^{2}$ of the rare earth elements in nitride films. This value was used to determine the film thickness and stoichiometry. A reasonable agreement between the calculated film thickness with that of fitting the optical data was obtained. A magnetic transition temperature was determined by DC resistivity measurements and SQUID magnetometry. GdN was found to possess a Curie temperature of $50 \mathrm{~K}$, lower than the generally accepted value of $70 \mathrm{~K}$. The $\mathrm{T}_{C}$ for DyN was around $20 \mathrm{~K}$.

To resolve the long outstanding question on the optical energy gap of the RENs, a Fourier Transform Infrared spectrometer (FTIR) and a conventional UV/Vis spectrometer were used. Transmission and reflection spectra on stoichiometric, polycrystalline rare earth nitrides were collected in the range of photon energy $0.5-5.0 \mathrm{eV}$. Due to multiple 
transmission and reflection of light from the air/cap/film/substrate interfaces, interference fringes appear on the resulting transmission/reflection spectra masking the true absorption edge of the material. To determine the optical energy gap accurately, these interference fringes were resolved by producing simulated reflection/transmission spectra with the help of computer software. A good agreement was found between experimental and fitted spectra. The optical energy gap and complex index of refraction were, thus, determined for GdN, DyN, SmN and EuN.

The experimentally determined direct energy gap for $\mathrm{GdN}, 1.30 \pm 0.05 \mathrm{eV}$ coincides well with the theoretically estimated ${ }^{1}$ value of $1.3 \pm 0.03 \mathrm{eV}$. Similar agreement ${ }^{2}$ is found for DyN, $\left(\mathrm{E}_{g}=1.2 \pm 0.05 \mathrm{eV}\right)$. For $\mathrm{SmN}$, however, the energy gap is underestimated by the theory $^{2}$, being nearly $0.5 \mathrm{eV}$ smaller than the experimental $\mathrm{E}_{g}=1.3 \pm 0.1 \mathrm{eV}$. A QSGW band structure calculation ${ }^{3}$ tuned to the experimental direct energy gap of $0.97 \pm 0.05$ produced a semiconducting solution for EuN. The index of refraction for GdN was found to be overestimated by the theory. There were no experimental or theoretical reports on the refractive index of DyN. However, given that the band structures of GdN and DyN are similar, it is quite likely that the refractive index for both compounds is similar i.e. approximately $2.0 \pm 0.1$.

The temperature dependent transmission spectra were determined for GdN and DyN in the photon energy range $0.5-2.1 \mathrm{eV}$. For GdN, while the fundamental absorption edge shifted towards the red part of the energy spectrum on lowering the temperature, a second absorption edge in the $k$ spectrum appeared at a higher photon energy. This was attributed to the spin - splitting of the conduction and the valence bands. The average energy gaps between the majority spin states, $0.85 \pm 0.05 \mathrm{eV}$, and for the minority states, $1.75 \pm 0.05 \mathrm{eV}$, concur well with the band structure of GdN calculated ${ }^{1}$ using the LSDA+ $U$ approximation, with a chosen value of Coulomb parameter $\mathrm{U}_{d}=8.0 \mathrm{eV}$. 


\subsection{Future Possibilities and Challenges}

The discussion presented above makes is clear that, although some highly interesting questions remain, a consensus is emerging among different experimental studies as well as theory and experiment. The series of rare earth nitrides contain several ferromagnetic semiconductors with direct energy gaps in the energy region $0.90 \mathrm{eV}$ to $1.30 \mathrm{eV}$ which make them of considerable interest for use in future spintronic devices. The results of section 5.2 show that GdN experiences a splitting of the electronic spin states below the Curie temperature. The exchange splitting of the conduction and the valence bands makes the direct energy gap at the $\mathrm{X}$ point spin dependent. The energy gap difference between the opposite spins makes this material a possible candidate for spin filtering devices, where the electronic charge of one type of spin flows while the other is blocked.

Improving the material quality is another immediate issue to address. At present, even the highest quality samples possess significant defect densities, nitrogen vacancies and grain boundaries which have significant affects on the physical properties of rare earth nitrides.

In all, the results presented in this experimental work on the optical parameters of rare earth nitride systems are of primary importance. The data provide a better understanding of the electronic structure of rare earth mononitrides with potential use in spintronic device fabrication. 


\section{Bibliography}

1. Trodahl, H. J.; Preston, A. R. H.; Zhong, J.; Ruck, B. J.; Strickland, N. M.; Mitra, C.; Lambrecht, W. R. L. Physical Review B 2007, 76, 085211.

2. Larson, P.; Lambrecht, W. R. L.; Chantis, A.; van Schilfgaarde, M. Physical Review B 2007, 75, 045114.

3. Richter, J. H. et al. Physical Review B 2011, 84, 235120.

4. Kikkawa, J. M.; Awschalom, D. D. Nature 1999, 397, 139-141.

5. Sih, V.; Johnston-Halperin, E.; Awschalom, D. D. Proceedings of IEEE 2003, 91, $752-760$.

6. Golovach, V. N.; Loss, D. Semiconductor Science and Technology 2002, 17, 355.

7. Ball, P. Nature 2000, 404, 918-920.

8. Daughton, J. Proceedings of IEEE 2003, 91, 681-686.

9. Katti, R. Proceedings of IEEE 2003, 91, 687-702.

10. Tehrani, S.; Slaughter, J. M.; Deherrera, M.; Engel, B. N.; Rizzo, N. D.; Salter, J.; Durlam, M.; Dave, R. W.; Janesky, J.; Butcher, B.; Smith, K.; Grynkewich, G. Proceedings of IEEE 2003, 91, 703-714.

11. Awschalom, D. D.; Loss, D.; Samarth, N. Semiconductor Spintronics and Quantum Computation; Springer: Berlin, 2002.

12. Parkin, S. Proceedings of IEEE 2003, 91, 661-680.

13. Baibich, M. N.; Broto, J. M.; Fert, A.; Van Dau, F. N.; Petroff, F.; Etienne, P.; Creuzet, G.; Friederich, A.; Chazelas, J. Physical Review Letters 1988, 61, 24722475 .

14. Binasch, G.; Grünberg, P.; Saurenbach, F.; Zinn, W. Physical Review B 1989, 39, 4828-4830.

15. Matsukura, F.; Ohno, H.; Dietl, T. Handbook of Magnetic Materials; Elsevier: Amsterdam, 2002; Vol. 14.

16. Ohno, H. Journal of Magnetism and Magnetic Materials 1999, 200, 110-129.

17. Prinz, G. A. Science 1998, 282, 1660-1663.

18. Wolf, S. A.; Awschalom, D. D.; Buhrman, R. A.; Daughton, J. M.; von Molnár, S.; Roukes, M. L.; Chtchelkanova, A. Y.; Treger, D. M. Science 2001, 294, 1488-1495.

19. Ziese, M.; Thornton, M. J. Spin Electronics; Springer, 2001.

20. Ivanov, V. A.; Aminov, T. G.; Novotortsev, V. M.; Kalinnikov, V. T. Russian Chemical Bulletin 2004, 53, 2357-2405.

21. Awschalom, D.; Samarth, N. Physics 2001, 2, 50.

22. Datta, S.; Das, B. Applied Physics Letters 1990, 56, 665-667. 
23. Schliemann, J.; Egues, J. C.; Loss, D. Physical Review Letters 2003, 90, 146801.

24. Egues, J. C.; Burkard, G.; Loss, D. Applied Physics Letters 2003, 82, 2658-2660.

25. Bandyopadhyay, S.; Cahay, M. Introduction to Spintronics; CRC Press, 2008.

26. Žutić, I.; Fabian, J.; Das Sarma, S. Reviews of Modern Physics 2004, 76, 323-410.

27. Ovcharenko, V. I.; Sagdeev, R. Z. Russian Chemical Reviews 1999, 68, 345-363.

28. Drexler, K. E. Nanosystems: Molecular Machinery, Manufacturing, and Computation; Wiley: New York, 1992.

29. Drexler, K. Molecular Engineering - An Approach to the Development of General Capabilities for Molecular Manipulation, 1981.

30. Buchachenko, A. L.; Molin, Y. N.; Sagdeev, R. Z.; Salikhov, K. M.; Frankevich, E. L. Soviet Physics Uspekhi 1987, 30, 79.

31. Buchachenko, A. L. Russian Chemical Reviews 2003, 72, 375-391.

32. Bonanni, A. Semiconductor Science and Technology 2007, 22, R41.

33. Ohno, H.; Munekata, H.; Penney, T.; von Molnár, S.; Chang, L. L. Physical Review Letters 1992, 68, 2664-2667.

34. Munekata, H.; Ohno, H.; von Molnar, S.; Segmüller, A.; Chang, L. L.; Esaki, L. Physical Review Letters 1989, 63, 1849-1852.

35. Ohno, H.; Shen, A.; Matsukura, F.; Oiwa, A.; Endo, A.; Katsumoto, S.; Iye, Y. Applied Physics Letters 1996, 69, 363-365.

36. Frazier, R.; Thaler, G.; Overberg, M.; Gila, B.; Abernathy, C. R.; Pearton, S. J. Applied Physics Letters 2003, 83, 1758-1760.

37. Park, M. C.; Huh, K. S.; Myoung, J. M.; Lee, J. M.; Chang, J. Y.; Lee, K. I.; Han, S. H.; Lee, W. Y. Solid State Communications 2002, 124, 11-14.

38. Liu, H. X.; Wu, S. Y.; Singh, R. K.; Gu, L.; Smith, D. J.; Newman, N.; Dilley, N. R.; Montes, L.; Simmonds, M. B. Applied Physics Letters 2004, 85, 4076-4078.

39. Shon, Y.; Kwon, Y. H.; Park, Y. S.; Yuldashev, S. U.; Lee, S. J.; Park, C. S.; Chung, K. J.; Yoon, S. J.; Kim, H. J.; Lee, W. C.; Fu, D. J.; Kang, T. W.; Fan, X. J.; Park, Y. J.; Oh, H. T. Journal of Applied Physics 2004, 95, 761-763.

40. Lee, J.-H.; Choi, I.-H.; Shin, S.; Lee, S.; Lee, J.; Whang, C.; Lee, S.-C.; Lee, K.-R.; Baek, J.-H.; Chae, K. H.; Song, J. Applied Physics Letters 2007, 90, 032504.

41. Hashimoto, M.; Zhou, Y.-K.; Kanamura, M.; Asahi, H. Solid State Communications 2002, 122, 37-39.

42. Lee, J. S.; Lim, J. D.; Khim, Z. G.; Park, Y. D.; Pearton, S. J.; Chu, S. N. G. Journal of Applied Physics 2003, 93, 4512-4516.

43. Xueqiong, S.; Li, W.; Jiangbo, C.; Xiaojing, W.; XinPing, Z.; Wang, R. P. Journal of Physics D: Applied Physics 2011, 44, 265002. 
44. Abdul, M.; Rehana, S.; Husnain, G.; Akbar, A. Journal of Physics D: Applied Physics 2009, 42, 135401.

45. Teraguchi, N.; Suzuki, A.; Nanishi, Y.; Zhou, Y.-K.; Hashimoto, M.; Asahi, H. Solid State Communications 2002, 122, 651-653.

46. Choi, S. W.; Zhou, Y. K.; Emura, S.; Lee, X. J.; Teraguchi, N.; Suzuki, A.; Asahi, H. Physica Status Solidi (c) 2006, 3, 2250-2253.

47. Dhar, S.; Kammermeier, T.; Ney, A.; Perez, L.; Ploog, K. H.; Melnikov, A.; Wieck, A. D. Applied Physics Letters 2006, 89, 062503.

48. Han, S. Y.; Hite, J.; Thaler, G. T.; Frazier, R. M.; Abernathy, C. R.; Pearton, S. J.; Choi, H. K.; Lee, W. O.; Park, Y. D.; Zavada, J. M.; Gwilliam, R. Applied Physics Letters 2006, 88, 042102.

49. Pérez, L.; Lau, G. S.; Dhar, S.; Brandt, O.; Ploog, K. H. Physical Review B 2006, 74, 195207.

50. Overberg, M.; Lee, K. N.; Abernathy, C. R.; Pearton, S. J.; Hobson, W. S.; Wilson, R. G.; Zavada, J. M. Materials Science and Engineering: B 2001, 81, 150152.

51. Zhou, Y. K.; Kim, M. S.; Teraguchi, N.; Suzuki, A.; Nanishi, Y.; Asahi, H. Physica Status Solidi (b) 2003, 240, 440-442.

52. Bang, H.; Sawahata, J.; Piao, G.; Tsunemi, M.; Yanagihara, H.; Kita, E.; Akimoto, K. Physica Status Solidi (c) 2003, 0, 2874-2877.

53. Ugolini, C.; Nepal, N.; Lin, J. Y.; Jiang, H. X.; Zavada, J. M. Applied Physics Letters 2006, 89, 151903.

54. Zajac, M.; Gosk, J.; Grzanka, E.; Kaminska, M.; Twardowski, A.; Strojek, B.; Szyszko, T.; Podsiadlo, S. Journal of Applied Physics 2003, 93, 4715-4717.

55. Dhar, S.; Brandt, O.; Trampert, A.; Friedland, K. J.; Sun, Y. J.; Ploog, K. H. Physical Review B 2003, 67, 165205.

56. Haider, M. B.; Constantin, C.; Al-Brithen, H.; Yang, H.; Trifan, E.; Ingram, D.; Smith, A. R.; Kelly, C. V.; Ijiri, Y. Journal of Applied Physics 2003, 93, 5274-5281.

57. Mauger, A.; Godart, C. Physics Reports 1986, 141, 51-176.

58. Kasuya, T.; Yanase, A. Reviews of Modern Physics 1968, 40, 684-696.

59. Dietl, T. Semiconductor Science and Technology 2002, 17, 377.

60. Watchter, P. CRC Critical Reviews in Solid State Science 1972, 3, 189.

61. Holtzberg, F.; Molnar, S. v.; Coey, J. M. D. The Handbook on Semiconductors; 1980.

62. Esaki, L.; Stiles, P. J.; Molnar, S. v. Physical Review Letters 1967, 19, 852-854.

63. Kalinnikov, V. T.; Aminov, T. G.; Novotortsev, V. M. Inorganic Materials 2003, 39, 997-1012.

64. Coey, J. M. D.; Viret, M.; von Molnár, S. Advances in Physics 1999, 48, 167-293. 
65. Tokura, Y. Clossal Magnetoresistive Oxides; Gordon and Breach: London, 2000.

66. Manyala, N.; Sidis, Y.; DiTusa, J. F.; Aeppli, G.; Young, D. P.; Fisk, Z. Nat Mater 2004, 3, 255-262.

67. Cullity, B. D. Introduction to Magnetic Materials; Addison-Wesley, 1972.

68. Duan, C.-G.; Sabirianov, R. F.; Mei, W. N.; Dowben, P. A.; Jaswal, S. S.; Tsymbal, E. Y. Journal of Physics: Condensed Matter 2007, 19, 315220.

69. Ruck, B. J. Spintronic Potential of Rare-Earth Nitrides and related materials. In Nanomagnetism and Spintronics; World Scietific, 2008; pp 193-221.

70. Gschneidner, K.; Beaudry, B. Rare Earth Compounds. In CRC Handbook of Thermoelectrics; CRC Press, 1995.

71. Natali, F.; Ruck, B. J.; Plank, N. O. V.; Trodahl, H. J.; Granville, S.; Meyer, C.; Lambrecht, W. R. L. arXiv:1208.2410 2012.

72. Olcese, G. L. Journal of Physics F: Metal Physics 1979, 9, 569.

73. Li, D. X.; Haga, Y.; Shida, H.; Suzuki, T.; Kwon, Y. S.; Kido, G. Journal of Physics: Condensed Matter 1997, 9, 10777.

74. Gerlach, J. W.; Mennig, J.; Rauschenbach, B. Applied Physics Letters 2007, 90, 061919.

75. Scarpulla, M. A.; Gallinat, C. S.; Mack, S.; Speck, J. S.; Gossard, A. C. Journal of Crystal Growth 2009, 311, 1239-1244.

76. Petit, L.; Tyer, R.; Szotek, Z.; Temmerman, W. M.; Svane, A. New Journal of Physics 2010, 12, 113041.

77. Dismukes, J. P.; Yim, W. M.; Tietjen, J. J.; Novak, R. E. Journal of Crystal Growth 1971, 9, 295.

78. Sclar, N. Journal of Applied Physics 1962, 33, 2999-3002.

79. Wilkinson, M. K.; Child, H. R.; Cable, J. W.; Wollan, E. O.; Koehler, W. C. Journal of Applied Physics 1960, 31, S358-S359.

80. Hulliger, F. Chapter 33 Rare earth pnictides. In Handbook on the Physics and Chemistry of Rare Earths; Karl A. Gschneidner, J., LeRoy, E., Eds.; Elsevier, 1979; Vol. 4, pp 153-236.

81. Vogt, O.; Mattenberger, K. Magnetic measurements on rare earth and and actinide monopnictides and monchalcogenides; Elsevier: Amsterdam, 1993; Vol. 17.

82. Fitzmaurice, J. C.; Hector, A.; Rowley, A. T.; Parkin, I. P. Polyhedron 1994, 13, 235-240.

83. Dismukes, Y. W. e. a., JP RCA Review 1970.

84. Kaldis, E.; Zurcher, C. Helvetica Physica Acta 1974, 47, 421-423.

85. E. Kaldis, B. F. E. J. A. W., B. Steinmann The Rare Earths in Modern Science and Technology; Plenum: New York, 1982; Vol. 3. 
86. Travaglini, G.; Marabelli, F.; Monnier, R.; Kaldis, E.; Wachter, P. Physical Review B 1986, 34, 3876-3882.

87. Degiorgi, L.; Bacsa, W.; Wachter, P. Physical Review B 1990, 42, 530.

88. Imamura, H.; Imahashi, T.; Zaimi, M.; Sakata, Y. Journal of Alloys and Compounds 2008, 451, 636-639.

89. Ruck, B. J.; Natali, F.; Plank, N. O. V.; Le, B. D.; Azeem, M.; Alfheid, M.; Meyer, C.; Trodahl, H. J. Physica B: Condensed Matter 2011, 407, 2954-2956.

90. Natali, F.; Plank, N. O. V.; Galipaud, J.; Ruck, B. J.; Trodahl, H. J.; Semond, F.; Sorieul, S.; Hirsch, L. Journal of Crystal Growth 2010, 312, 3583-3587.

91. Natali, F.; Ludbrook, B.; Galipaud, J.; Plank, N.; Granville, S.; Preston, A.; Do, B. L.; Richter, J.; Farrell, I.; Reeves, R.; Durbin, S.; Trodahl, J.; Ruck, B. Physica Status Solidi (c) 2012, 9, 605-608.

92. Ruck, B. J.; Trodahl, H. J.; Richter, J. H.; Cezar, J. C.; Wilhelm, F.; Rogalev, A.; Antonov, V. N.; Le, B. D.; Meyer, C. Physical Review B 2011, 83, 174404, PRB.

93. Shimomoto, K.; Ohta, J.; Fujii, T.; Ohba, R.; Kobayashi, A.; Oshima, M.; Fujioka, H. Journal of Crystal Growth 2009, 311, 4483-4485.

94. Ludbrook, B. M.; Farrell, I. L.; Kuebel, M.; Ruck, B. J.; Preston, A. R. H.; Trodahl, H. J.; Ranno, L.; Reeves, R. J.; Durbin, S. M. Journal of Applied Physics 2009, 106, 063910.

95. III, R. M. O.; Pearson, J. E.; Sowers, C. H.; Bader, S. D. Journal of Applied Physics 1998, 84, 940-944.

96. Farrell, I. L.; Reeves, R. J.; Preston, A. R. H.; Ludbrook, B. M.; Downes, J. E.; Ruck, B. J.; Durbin, S. M. Applied Physics Letters 2010, 96, 071914.

97. Yoshitomi, H.; Kitayama, S.; Kita, T.; Wada, O.; Fujisawa, M.; Ohta, H.; Sakurai, T. Physica Status Solidi (c) 2011, 8, 488-490.

98. Yoshitomi, H.; Kitayama, S.; Kita, T.; Wada, O.; Fujisawa, M.; Ohta, H.; Sakurai, T. Physical Review B 2011, 83, 155202.

99. Yoshitomi, H.; Vidyasagar, R.; Kitayama, S.; Kita, T.; Ohta, H.; Okubo, S.; Fukuoka, Y.; Sakurai, T. Applied Physics Letters 2012, 101, 072403-5.

100. Azeem, M.; Ruck, B. J.; Le, B. D.; Warring, H.; Trodahl, H. J.; Strickland, N. M.; Koo, A.; Goian, V.; Kamba, S. Journal of Applied Physics 2013, 113, 203509.

101. Azeem, M.; Ruck, B. J.; Strickland, N. M.; Koo, A.; Trodahl, H. J. unpublished 2012.

102. Lee, T.-Y.; Gall, D.; Shin, C.-S.; Hellgren, N.; Petrov, I.; Greene, J. E. Journal of Applied Physics 2003, 94, 921-927.

103. Natali, F.; Plank, N. O. V.; Stephen, J.; Azeem, M.; Trodahl, H. J.; Ruck, B. J.; Hirsch, L. Journal of Physics D: Applied Physics 2011, 44, 135404.

104. Leuenberger, F.; Parge, A.; Felsch, W.; Fauth, K.; Hessler, M. Physical Review B 2005, 72, 014427, PRB. 
105. Thiede, T. B.; Krasnopolski, M.; Milanov, A. P.; de los Arcos, T.; Ney, A.; Becker, H.-W.; Rogalla, D.; Winter, J.; Devi, A.; Fischer, R. A. Chemistry of Materials 2011, 23, 1430-1440, doi: 10.1021/cm102840v.

106. Fang, Z.; Williams, P. A.; Odedra, R.; Jeon, H.; Potter, R. J. Journal of Crystal Growth 2012, 338, 111-117.

107. Busch, G. Journal of Applied Physics 1967, 38, 1386-1394.

108. Bommeli, F.; Degiorgi, L.; Wachter, P. Journal of Magnetism and Magnetic Materials 1995, 140 - 144, Part 2, 1159-1160.

109. Didchenko, R.; Gortsema, F. P. Journal of Physics and Chemistry of Solids 1963, $24,863-870$.

110. Degiorgi, L.; Teraoka, S.; Compagnini, G.; Wachter, P. Physical Review B 1993, 47, 5715.

111. Aerts, C. M.; Strange, P.; Horne, M.; Temmerman, W. M.; Szotek, Z.; Svane, A. Physical Review B 2004, 69, 045115.

112. Preston, A. Electronic Structure of Rare Earth Nitrides, Ph.D. thesis, 2009.

113. Martin, R. P. Electronic Structure: Basic Theory and Practical Methods; Cambridge University Press, 2004.

114. Larson, P.; Lambrecht, W. R. L. Physical Review B 2006, 74, 085108.

115. Hasegawa, A.; Yanase, A. Journal of the Physical Society of Japan 1977, 42, 492498.

116. Lambrecht, W. R. L. Physical Review B 2000, 62, 13538.

117. Ghosh, D. B.; De, M.; De, S. K. Physical Review B 2005, 72, 045140.

118. Larson, P.; Lambrecht, W. R. L. Journal of Physics: Condensed Matter 2006, 18, 11333.

119. Doll, K. Journal of Physics: Condensed Matter 2008, 20, 075214.

120. Mitra, C.; Lambrecht, W. R. L. Physical Review B 2008, 78, 195203.

121. Cutler, R. A.; Lawson, A. W. Journal of Applied Physics 1975, 46, 2739-2744.

122. Schneemeyer, L. F.; Dover, R. B. v.; Gyorgy, E. M. Journal of Applied Physics 1987, $61,3543-3545$.

123. Schumacher, D. P.; Wallace, W. E. Journal of Applied Physics 1965, 36, 984-985.

124. McGuire, T. R.; Gambino, R. J.; Pickart, S. J.; Alperin, H. A. Journal of Applied Physics 1969, 40, 1009-1010.

125. Li, D. X.; Haga, Y.; Shida, H.; Suzuki, T. Physica B: Condensed Matter 1994, 199 $-200,631-633$.

126. Kasuya, T.; Li, D. X. Journal of Magnetism and Magnetic Materials 1997, 167, L1-L6. 
127. Xiao, J. Q.; Chien, C. L. Physical Review Letters 1996, 76, 1727-1730.

128. Khazen, K.; von Bardeleben, H. J.; Cantin, J. L.; Bittar, A.; Granville, S.; Trodahl, H. J.; Ruck, B. J. Physical Review B 2006, 74, 245330.

129. Gambino, R. J.; McGuire, T. R.; Alperin, H. A.; Pickart, S. J. Journal of Applied Physics 1970, 41, 933-934.

130. Senapati, K.; Fix, T.; Vickers, M. E.; Blamire, M. G.; Barber, Z. H. Physical Review B 2011, 83, 014403.

131. Wachter, P.; Kaldis, E. Solid State Communications 1980, 34, 241-244.

132. Senapati, K.; Fix, T.; Vickers, M. E.; Blamire, M. G.; Barber, Z. H. Journal of Physics: Condensed Matter 2010, 22, 302003.

133. Duan, C.-G.; Sabiryanov, R. F.; Mei, W. N.; Dowben, P. A.; Jaswal, S. S.; Tsymbal, E. Y. Applied Physics Letters 2006, 88, 182505.

134. Rhyne, J.; McGuire, T. Magnetics, IEEE Transactions on 1972, 8, 105-130.

135. Wachter, P.; Kaldis, E.; Hauger, R. Physical Review Letters 1978, 40, 1404-1407.

136. Holtzberg, F.; McGuire, T. R.; Methfessel, S.; Suits, J. C. Physical Review Letters 1964, 13, 18-21.

137. Plank, N. O. V.; Natali, F.; Galipaud, J.; Richter, J. H.; Simpson, M.; Trodahl, H. J.; Ruck, B. J. Applied Physics Letters 2011, 98, 112503.

138. Leuenberger, F.; Parge, A.; Felsch, W.; Baudelet, F.; Giorgetti, C.; Dartyge, E.; Wilhelm, F. Physical Review B 2006, 73, 214430.

139. Abdelouahed, S.; Alouani, M. Physical Review B 2007, 76, 214409.

140. Antonov, V. N.; Harmon, B. N.; Yaresko, A. N.; Shpak, A. P. Physical Review B 2007, 75, 184422.

141. Petukhov, A. G.; Lambrecht, W. R. L.; Segall, B. Physical Review B 1996, 53, 4324-4339.

142. Mitra, C.; Lambrecht, W. R. L. Physical Review B 2008, 78, 134421.

143. Meyer, C.; Ruck, B. J.; Zhong, J.; Granville, S.; Preston, A. R. H.; Williams, G. V. M.; Trodahl, H. J. Physical Review B 2008, 78, 174406.

144. Adachi, H.; Ino, H.; Miwa, H. Physical Review B 1999, 59, 11445-11449.

145. Ahn, K.; Pecharsky, V. K.; Gschneidner, J., K. A. Physical Review B 2007, 76, 014415 .

146. de Wijn, H. W.; van Diepen, A. M.; Buschow, K. H. J. Physical Review B 1973, 7, 524-533.

147. Preston, A. R. H.; Granville, S.; Housden, D. H.; Ludbrook, B.; Ruck, B. J.; Trodahl, H. J.; Bittar, A.; Williams, G. V. M.; Downes, J. E.; DeMasi, A.; Zhang, Y.; Smith, K. E.; Lambrecht, W. R. L. Physical Review B 2007, 76, 245120.

148. Johannes, M. D.; Pickett, W. E. Physical Review B 2005, 72, 195116. 
149. Ziese, M.; Thornton, M. J. Spin Electronics; Springer, 2001.

150. Nakagawa, T.; Sako, K.; Arakawa, T.; Yamamoto, T. A. Journal of Alloys and Compounds 2004, 364, 53-58.

151. Yamamoto, T. A.; Nakagawa, T.; Sako, K.; Arakawa, T.; Nitani, H. Journal of Alloys and Compounds 2004, 376, 17-22.

152. Dash, W. C.; Newman, R. Physical Review 1955, 99, 1151-1155.

153. Philipp, H. R.; Taft, E. A. Physical Review 1959, 113, 1002-1005.

154. Newman, R.; Chrenko, R. M. Physical Review 1959, 114, 1507-1513.

155. Cardona, M. Physical Review 1965, 140, A651-A655.

156. Aspnes, D. E.; Studna, A. A. Physical Review B 1983, 27, 985-1009.

157. Calvani, P.; Paolone, A.; Dore, P.; Lupi, S.; Maselli, P.; Medaglia, P. G.; Cheong, S. W. Physical Review B 1996, 54, R9592-R9595.

158. Zhang, X. Y.; Gall, D. Physical Review B 2010, 82, 045116.

159. Knyazev, Y. V.; Noskov, M. M. Physica Status Solidi (b) 1977, 80, 11-29.

160. Quemerais, A.; Loisel, B.; Jezequel, G.; Thomas, J.; Lemonnier, J. C. Journal of Physics F: Metal Physics 1981, 11, 293.

161. Jiles, D. C.; Staines, M. P. Physical Review B 1983, 28, 5746-5755.

162. Endriz, J. G.; Spicer, W. E. Physical Review B 1970, 2, 1466-1492.

163. Krizek, J.; Taylor, K. N. R. Journal of Physics F: Metal Physics 1975, 5, 774.

164. Jackson, C. Physical Review 1969, 178, 949-952.

165. Shalaan, E.; Schmitt, H. Optics Communications 2006, 260, 588-594.

166. Vidyasagar, R.; Kitayama, S.; Yoshitomi, H.; Kita, T.; Sakurai, T.; Ohta, H. Applied Physics Letters 2012, 100, 232410.

167. Xue, D.; Betzler, K.; Hesse, H. Journal of Physics: Condensed Matter 2000, 12, 3113.

168. Zhang, S. Journal of Rare Earths 1995, 13, 85-88.

169. Fox, M. Optical Properties of Solids; Oxford University Press: New York, 2010.

170. Bassani, F.; Parravicini, G. P. Electronic States and Optical Transitions in Solids; Pergamon Press, 1975; Vol. 8.

171. Wooten, F. Optical Properties of Solids; Academic Press: New York and London, 1972.

172. Heavens, O. Optical Properties of Thin Solid Films; Butterworths Scientific Publications.: London, 1955.

173. Palik, E. Handbook of Optical Constants of Solids; Academic: New York, 1985. 
174. Crook, A. W. J. Opt. Soc. Am. 1948, 38, 954-963.

175. Noe, T. TFCalc, 2011.

176. Sloan Technology Corporation Handbook of Thin Film Materials; Sloan Materials Division: California.

177. Cullity, B. D. Elements of X-ray Diffraction, 2nd ed.; Addison-Wesley Publication Company, INC, 1978.

178. Madsen, R. J. H.; C., I. Structure Determination from Powder Diffraction Data; Oxford University Press: New York, 2002.

179. Rubin, S.; Passell, T. O.; Bailey, E. Analytical Chemistry 1957.

180. Tesmer, J. R.; Nastasi, M. Handbook of Modern Ion Beam Materials Analysis; Materials Research Society: Pittsburgh, Pennsylvania, 1995.

181. Pauw, L. J. v. d. A Method of Measuring Specific Resistivity and Hall Effect of Discs of Arbitrary Shapes; Technical Report, 1958.

182. G.A. Vanasse, A. S. J.; Baker, D. J. Aspen International Conference on Fourier Spectroscopy, Massachusetts, 1970.

183. Griffiths, P. Chemical Infrared Fourier Transform Spectroscopy; John Wiley \& Sons, 1975.

184. Taylor, K. N. R.; Darby, M. I. Physics f Rare Earth Solids; Chapman and Hall Ltd.: London, 1972.

185. Junod, P.; Menth, A.; Vogt, O. Physik der Kondensierten Materie 1969, 8, 323-370.

186. Ohno, H. Science 2001, 291, 840-841.

187. Granville, S.; Meyer, C.; Preston, A. R. H.; Ludbrook, B. M.; Ruck, B. J.; Trodahl, H. J.; Paudel, T. R.; Lambrecht, W. R. L. Physical Review B 2009, 79, 054301.

188. Granville, S.; Ruck, B. J.; Budde, F.; Koo, A.; Pringle, D. J.; Kuchler, F.; Preston, A. R. H.; Housden, D. H.; Lund, N.; Bittar, A.; Williams, G. V. M.; Trodahl, H. J. Physical Review B 2006, 73, 235335.

189. Sycon Instrument STC-2002 Thin Film Thickness and Rate Controller: Users' Manual; 2003.

190. Deakin, M. R.; Buttry, D. A. Analytical Chemistry 1989, 61, 1147A-1154A.

191. Buttry, D. A. The Quartz Crystal Microbalance as an In Situ Tool for Electrochemistry. In Electrochemical Interfaces: Modern Techniques for In-Situ Interface Characterization; Abruna, H. D., Ed.; VCH Publishers, Inc.,: New York, 1991.

192. Sharma, A.; Nolting, W. Physical Review B 2010, 81, 125303.

193. Natali, F.; Ruck, B. J.; Trodahl, H. J.; Binh, D. L.; Vezian, S.; Damilano, B.; Cordier, Y.; Semond, F.; Meyer, C. Physical Review B 2013, 87, 035202. 
194. Duan, C.-g.; Sabiryanov, R. F.; Liu, J.; Mei, W. N.; Dowben, P. A.; Hardy, J. R. Physical Review Letters 2005, 94, 237201.

195. Nagaev, E. L. Physics of Magnetic Semiconductors; Izd Mir: Moscow, 1983.

196. Moskvin, A. S.; Loshkareva, N. N.; Sukhorukov, Y. P.; Sidorov, M. A.; Samokhvalov, A. A. Soviet Journal of Experimental and Theoretical Physics 1994, $78,518-532$.

197. Gilliland, S. J.; Sans, J. A.; Sánchez-Royo, J. F.; Almonacid, G.; GarcíaDomene, B.; Segura, A.; Tobias, G.; Canadell, E. Physical Review B 2012, 86, 155203.

198. Malerba, C.; Biccari, F.; Leonor Azanza Ricardo, C.; D’Incau, M.; Scardi, P.; Mittiga, A. Solar Energy Materials and Solar Cells 2011, 95, 2848-2854.

199. Punya, A.; Cheiwchanchamnagij, T.; Thiess, A.; Lambrecht, W. Materials Research Society (MRS) Fall Meeting. Materials Research Society (MRS) Fall Meeting, Boston, 2011.

200. Busch, G.; Wachter, P. Helvetica Physica Acta 1969, 42, 930.

201. van Schilfgaarde, M.; Kotani, T.; Faleev, S. Physical Review Letters 2006, 96, 226402. 